Article

\title{
Exclusively Breastfed Infant Microbiota Develops over Time and Is Associated with Human Milk Oligosaccharide Intakes
}

\author{
Ali Sadiq Cheema ${ }^{1}\left(\mathbb{D}\right.$, Michelle Louise Trevenen ${ }^{2}$, Berwin Ashoka Turlach ${ }^{2} \mathbb{D}$, Annalee June Furst ${ }^{3,4}(\mathbb{D}$, \\ Ana Sophia Roman ${ }^{3,4}$, Lars Bode ${ }^{3,4}$, Zoya Gridneva ${ }^{1}$ (D), Ching Tat Lai ${ }^{1}$ (D), Lisa Faye Stinson ${ }^{1}$ (D), \\ Matthew Scott Payne 5,6 and Donna Tracy Geddes 1,*
}

1 School of Molecular Sciences, The University of Western Australia, Crawley, WA 6009, Australia; alisadiq.cheema@research.uwa.edu.au (A.S.C.); zoya.gridneva@uwa.edu.au (Z.G.); ching-tat.lai@uwa.edu.au (C.T.L.); lisa.stinson@uwa.edu.au (L.F.S.)

2 Centre for Applied Statistics, The University of Western Australia, Crawley, WA 6009, Australia; michelle.trevenen@uwa.edu.au (M.L.T.); berwin.turlach@uwa.edu.au (B.A.T.)

3 Larsson-Rosenquist Foundation Mother-Milk-Infant Center of Research Excellence, University of California San Diego, La Jolla, CA 92093, USA; aloeffler@health.ucsd.edu (A.J.F.); acroman@sdsu.edu (A.S.R.); lbode@health.ucsd.edu (L.B.)

4 Department of Pediatrics, University of California San Diego, La Jolla, CA 92093, USA

5 Division of Obstetrics and Gynaecology, School of Medicine, The University of Western Australia, Subiaco, WA 6008, Australia; matthew.payne@uwa.edu.au

6 Women and Infants Research Foundation, Subiaco, WA 6008, Australia

* Correspondence: donna.geddes@uwa.edu.au; Tel.: +61-8-6488-4467

\section{check for}

updates

Citation: Cheema, A.S.; Trevenen,

M.L.; Turlach, B.A.; Furst, A.J.;

Roman, A.S.; Bode, L.; Gridneva, Z.;

Lai, C.T.; Stinson, L.F.; Payne, M.S.;

et al. Exclusively Breastfed Infant

Microbiota Develops over Time and

Is Associated with Human Milk

Oligosaccharide Intakes. Int. J. Mol.

Sci. 2022, 23, 2804. https://doi.org/

$10.3390 /$ ijms 23052804

Academic Editor: Daniela Fiocco

Received: 2 February 2022

Accepted: 28 February 2022

Published: 3 March 2022

Publisher's Note: MDPI stays neutral with regard to jurisdictional claims in published maps and institutional affiliations.

Copyright: (C) 2022 by the authors. Licensee MDPI, Basel, Switzerland. This article is an open access article distributed under the terms and conditions of the Creative Commons Attribution (CC BY) license (https:// creativecommons.org/licenses/by/ $4.0 /)$.

\begin{abstract}
Temporal development of maternal and infant microbiomes during early life impacts shortand long-term infant health. This study aimed to characterize bacterial dynamics within maternal faecal, human milk (HM), infant oral, and infant faecal samples during the exclusive breastfeeding period and to document associations between human milk oligosaccharide (HMO) intakes and infant oral and faecal bacterial profiles. Maternal and infant samples $(n=10)$ were collected at 2-5, 30, 60, 90 and 120 days postpartum and the full-length $16 \mathrm{~S}$ ribosomal RNA (rRNA) gene was sequenced. Nineteen HMOs were quantitated using high-performance liquid chromatography. Bacterial profiles were unique to each sample type and changed significantly over time, with a large degree of intraand inter-individual variation in all sample types. Beta diversity was stable over time within infant faecal, maternal faecal and HM samples, however, the infant oral microbiota at day 2-5 significantly differed from all other time points (all $p<0.02$ ). HMO concentrations and intakes significantly differed over time, and HMO intakes showed differential associations with taxa observed in infant oral and faecal samples. The direct clinical relevance of this, however, is unknown. Regardless, future studies should account for intakes of HMOs when modelling the impact of HM on infant growth, as it may have implications for infant microbiota development.
\end{abstract}

Keywords: maternal faecal; human milk; human milk oligosaccharides; human milk bacteria; infant oral; infant faecal; microbiome; 16S rRNA gene; breastfeeding; body composition; intake; concentration; lactation

\section{Introduction}

The maternal gut and human milk (HM) microbiota contribute to bacterial colonization of the infant gut, which in turn influences both short- and long-term infant health and development [1,2]. Aberrations to the early-life gut microbiota have been linked to various disorders, such as obesity [3], type 1 diabetes [4], allergies [5], asthma [6], and neurological diseases [7]. Early nutrition is a key factor in directing the composition and function of the infant gut microbiome [8], with breastfeeding being the most significant factor associated with infant gut bacterial structure and function in early life [9]. Therefore, it is important to 
document the temporal assembly of maternal and infant bacterial communities in the early postnatal period to better understand the foundations for life-long health.

The infant gut microbiome undergoes temporal compositional changes during early life and by 5 years of age is still developing, having not assumed diversity and composition similar to the adult gut [10]. During the first week of life, the infant gut bacterial profile is mainly composed of facultative anaerobes and obligate anaerobes, which are eventually replaced by strict anaerobes as the gut environment shifts in oxygenation [11-15]. HM consumption shifts the infant gut microbiome to a state dominated by Bifidobacterium sp. and lactic acid bacteria [16]. The commencement of solid foods again alters the gut microbiome, introducing typical adult gut genera such as Bacteroides, Prevotella, Ruminococcus, and Clostridium [16,17]. Although previous studies have characterized the development of the early-life gut microbiota, to date, no such study has been performed in an Australian cohort. This is important, as Australia is a geographically isolated continent, and both infant and adult microbiomes have been shown to vary geographically [18-21].

In addition to the infant gut, the development of the infant oral cavity is of interest, due to its contribution to oral health and its potential contribution to both the HM and infant gut microbiomes [13,22-24]. The acquisition of certain early oral microbiome bacteria such as Streptococcus mutans and Veillonella sp. has been associated with the development of periodontitis and dental caries $[25,26]$. During the first three months of life, certain taxa including Streptococcus mitis, Rothia mucilaginosa, Veillonella parvula, Streptococcus salivarius, Gemella haemolysans and Veillonella HB016 dominate the infant oral microbiota $[24,27,28]$. Changes in bacterial composition within the oral community are associated with feeding methods [28,29], tooth eruption [30] and introduction of solids [28]. Further, bacterial richness and diversity increase over the first 7 years of life $[27,28,31]$. However, a clear outline of the development of the infant oral microbiota during the exclusive breastfeeding period is still not well-documented.

The maternal microbiome is the primary donor of bacteria to the infant microbiome [13,32,33]. Studies have demonstrated vertical transmission of specific bacterial strains from the maternal to the infant gut [12,13,34-37], including Bacteroides spp., Bifidobacterium spp. and Escherichia coli. Despite the importance of the maternal gut microbiome as a contributor to the infant gut microbiome, changes to the maternal gut microbiome during the early postnatal period have not been characterized. The maternal gut microbiome undergoes profound changes from the first to third trimester with increased abundance of Proteobacteria and Actinobacteria, decreased richness and increased beta diversity [38]. At one month postpartum, the gut microbiota is similar to that present in the third trimester [38]. One study, which was restricted in taxonomic depth, reported no effect of time on maternal gut bacterial community composition or diversity in the first six months postpartum [39], which suggests that the maternal gut microbiome does not revert to a pre-pregnancy state or change substantially during this time. Given the dramatic remodeling of the maternal gut microbiome during pregnancy, further work is needed to examine the postnatal trajectory of the maternal gut microbiome, particularly in the context of lactation, a time when maternal hormones are in an altered state [40].

In addition to the maternal gut, HM has been highlighted as a source of bacteria for the infant gut microbiome, with evidence of vertical transmission of bacteria to the breastfed infant, particularly Bifidobacterium spp. [34-37]. Two to eighteen bacterial taxa have been reported to form the core HM community [14,18,41-43], with Staphylococcus $\mathrm{sp}$. and Streptococcus sp. typically dominating profiles. Lactation stage, particularly the transition from colostrum to mature milk has been associated with a change in bacterial composition $[14,19,44,45]$. However, some studies report relatively constant HM bacterial profiles over the first six months postpartum [24,41,46-48], with the exception of less abundant genera including Veillonella sp., Leptotrichia sp., Prevotella sp. and Granulicatella sp., which tend to shift over time $[46,47]$. While efforts have been made to characterize the HM microbiome, most of the previous studies are disadvantaged by a major confounder, which is the inclusion of infants fed with complementary foods and/or formula $[24,42,46,48]$. 
Therefore, studies focusing on exclusively breastfeeding dyads are required to negate the influence of formula and truly characterize the longitudinal development and/or stability of the HM microbiome during the exclusive breastfeeding period.

While a small number of bacterial taxa are vertically transmitted from mother to infant via HM [34-37], other HM components are also likely to contribute to the development of the infant gut microbiome. In particular, HM oligosaccharides (HMOs) are of great interest due to their potential to shape HM and infant oral/gut bacterial profiles [49]. HMOs are prebiotic agents that stimulate the growth of specific bacteria, such as Bifidobacterium spp., which are the dominant taxa in the breastfed infant gut [50,51]. Additionally, Bacteroides sp. and Streptococcus sp. have been shown to be able to metabolize HMOs [52,53]. Both positive and negative correlations between $\mathrm{HMO}$ concentrations and the relative abundance of certain gut bacteria, including Bifidobacterium sp. and Bacteroides sp., have been reported [53-57]; however, no previous study has examined the role of HMO daily intakes in shaping the infant oral and gut microbiome. To understand the influence of HMOs on the development of the infant microbiota, an integrated longitudinal analysis of HMO intakes and microbiota composition is needed.

Therefore, the aims of this longitudinal cohort study were to characterize the temporal development of the maternal faecal, HM, infant oral and infant faecal microbiomes over the first four months of life, to track the longitudinal variability of HMOs over this same time period and to determine associations between daily intakes of HMOs and infant oral and faecal bacterial profiles.

\section{Results}

\subsection{Participant Characteristics}

All mothers participating in the current study were Caucasian, had delivered vaginally, had not taken antibiotics, and were exclusively breastfeeding at all time points. Demographics of the 10 mother-infant dyads are shown in Table 1.

Table 1. Maternal and infant characteristics $(n=10)$.

\begin{tabular}{ll}
\hline Characteristics $(\boldsymbol{n}=\mathbf{1 0})$ & Mean \pm SD (Min-Max) or $\boldsymbol{n} \mathbf{( \% )}$ \\
\hline Maternal & $31.60 \pm 2.42(28-35)$ \\
Age at infant birth (years) & $2.50 \pm 0.50(2-3)$ \\
Parity & $4(40.0 \%)$ \\
Infant & $39.26 \pm 1.11(36.6-40.2)$ \\
Male $(n,(\%))$ & $3622.50 \pm 234.11(3320-4020)$ \\
Gestational age (weeks) & $51.55 \pm 1.39(49-54)$ \\
Birth weight (grams) & $837.80 \pm 131.17(580-1040)$ \\
Birth length (cm) &
\end{tabular}

\subsection{PacBio HiFi Sequencing Metrics}

The average number of circular consensus sequence (CCS passes) for two SMRT cells was 26 , and the minimum predicted sequencing accuracy was $99 \%$, meaning that our estimated sequencing error rate was less than $1 \%$.

\subsection{Temporal Development of Maternal Faecal Bacterial Profiles}

Within maternal faecal samples, nine OTUs were present at an average relative abundance of $>1 \%$ (Figure $1 \mathrm{~A}$ ) and these collectively represented $20.4-27.1 \%$ of the total bacterial profile. However, the relative abundance of these nine OTUs varied over time, with day $2-5$ presenting a different bacterial profile compared to all other time points. The day $2-5$ bacterial profile was dominated with OTU39 (Dialister invisus) $(9.2 \%)$ and OTU35 (Bacilli_c;RF39_o;RF39_fa;RF39_ge) $(7.8 \%)$, while day 30, 60 and 120 profiles were dominated with OTU23 (Phocaeicola vulgatus) and OTU27 (Akkermansia muciniphila) and day 90 dominated with OTU23 (P. vulgatus) and OTU06 (Escherichia coli). 

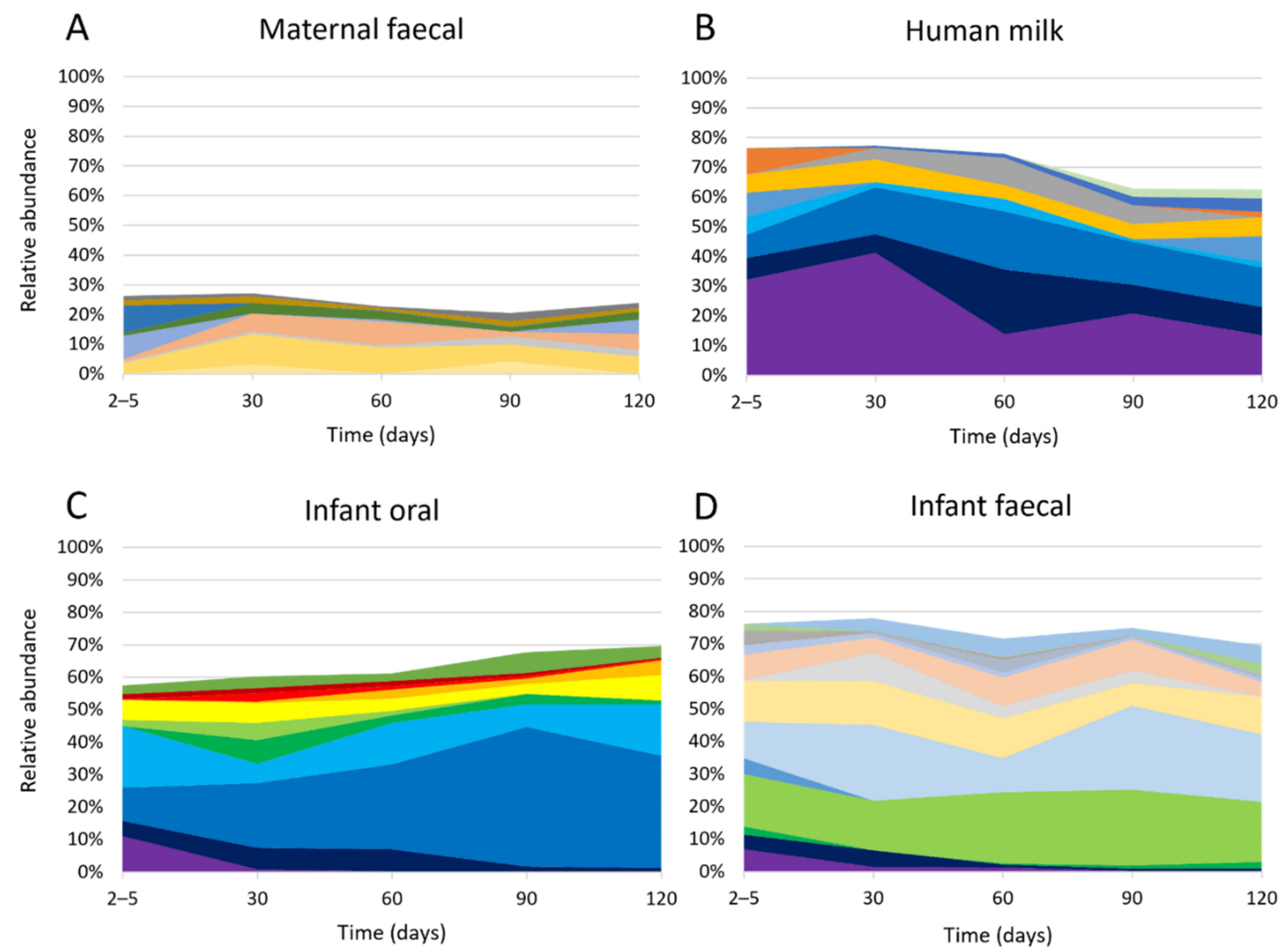

- OTU01 (Streptococcus mitis)

- OTU02 (Staphylococcus epidermidis)

= OTU03 (Bifidobacterium longum subsp. infantis)

\#TU04 (Bifidobacterium breve)

- OTU05 (Streptococcus salivarius)

OTU06 (Escherichia coli)

- OTU07 (Gemella haemolysans)

= OTU09 (Acinetobacter johnsonii)

= OTU08 (Cutibacterium acnes)

OTU11 (Haemophilus haemolyticus)

= OTU10 (Bifidobacterium pseudocatenulatum)

OTU12 (Streptococcus anginosus)

- OTU13 (Raoultella ornithinolytica)

- OTU14 (Veillonella nakazawae)

n OTU16 (Streptococcus lactarius)

n OTU15 (Moraxella osloensis)

n OTU17 (Klebsiella pneumoniae)

OTU18 (Veillonella sp. oral clone ASCB03)

n OTU19 (Rothia mucilaginosa)

= OTU20 (Bacteroides fragilis)

= OTU21 (Streptococcus agalactiae)

- OTU22 (Bergeyella sp.)

m OTU24 (Bifidobacterium longum)

= OTU27 (Akkermansia muciniphila)

= OTU25 (Parabacteroides distasonis)

- OTU32 (Haemophilus parainfluenzae)

- ОTU39 (Dialister invisus)

= 0 OTU35 (Bacilli_c;RF39_o;RF39_fa;RF39_ge)

= OTU43 (Faecalibacterium prausnitzii)

= OTU41 (Bacteroides dorei)

= OTU61 (Bacteroides uniformis)

- OTU64 (Bifidobacterium adolescentis)

Figure 1. The relative abundance of OTUs constituting $\geq 1 \%$ within each sample type. (A) Maternal faecal, (B) Human milk, (C) Infant oral and (D) Infant faecal samples.

The prevalence of six OTUs (OTU01 (S. mitis), OTU23 (P. vulgatus), OTU43 (Faecalibacterium prausnitzii), OTU64 (Bifidobacterium adolescentis), OTU82 (Oscillibacter sp.) and OTU107 (Romboutsia timonensis)) changed significantly over time (Figure 2A, Table A3).

Additionally, we observed a high level of inter-individual variability in OTU composition, with different taxa dominating different mother's bacterial profiles at different time points (Figure A1A). We also observed a high level of intra-individual changes in composition over time (Figure A1A). 


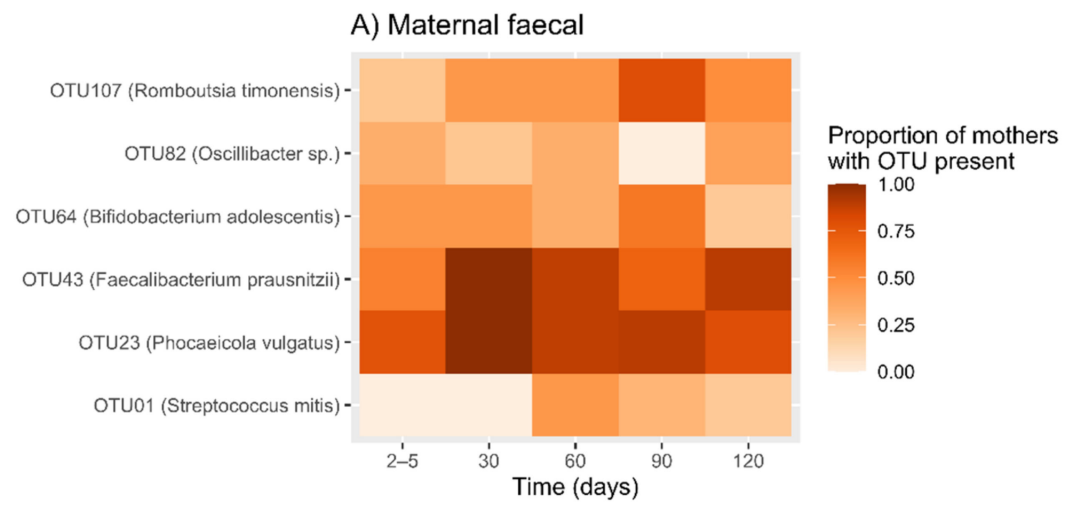

B) Human milk

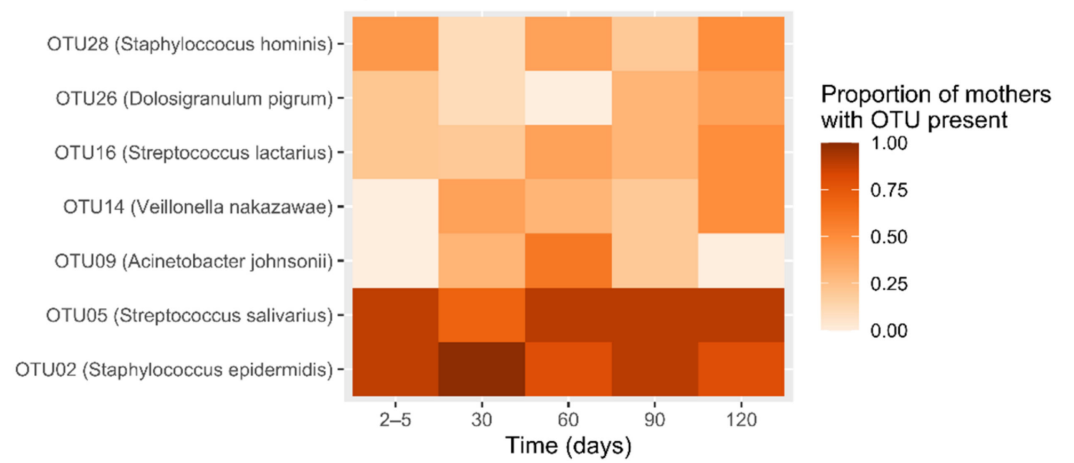

C) Infant oral

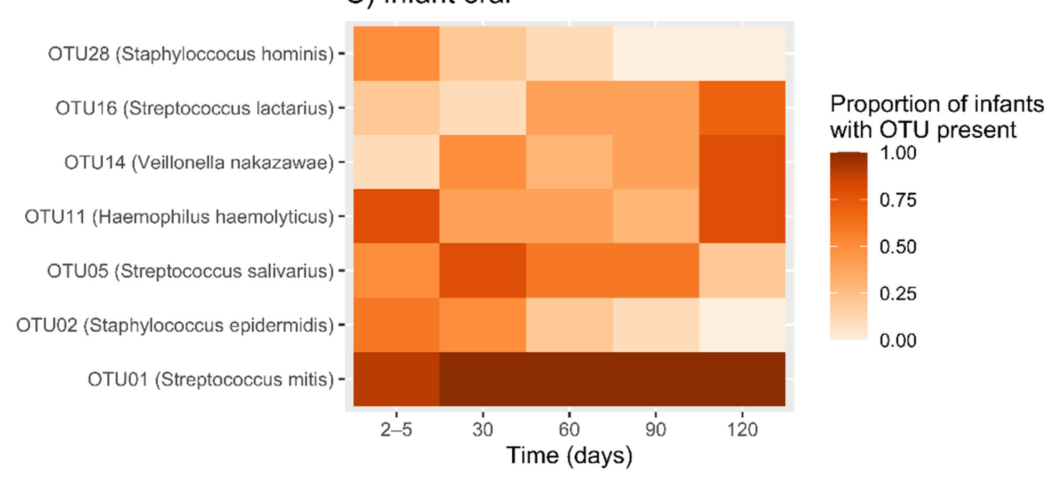

D) Infant faecal

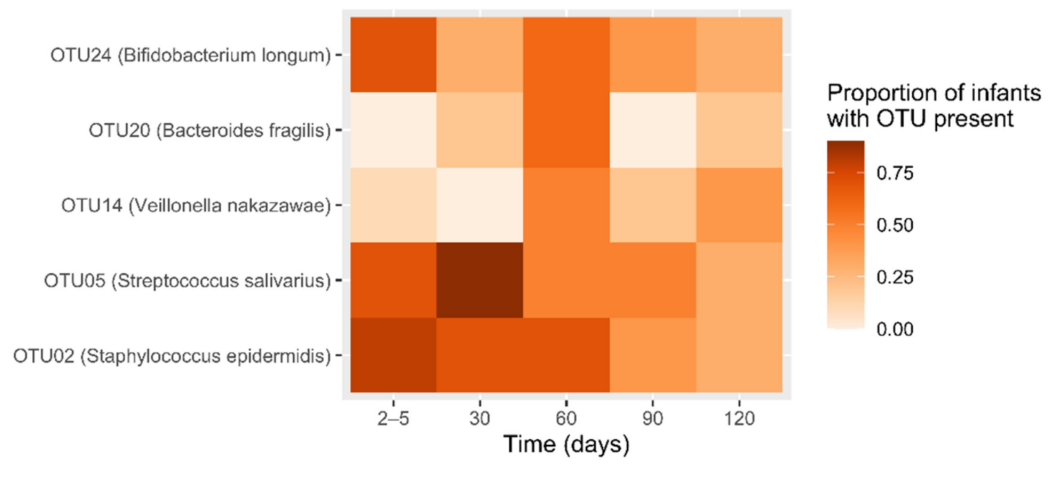

Figure 2. The proportion of mothers and infants at each time point for which an OTU was present. (A) Maternal faecal, (B) Human milk, (C) Infant oral and (D) Infant faecal samples. The darkest red/brown colour represents a proportion of 1, indicating that all mothers/infants had that OTU present at that time point. A white shaded box represents a proportion of 0 , indicating that no mothers/infants had that OTU present at that time point. 
Maternal faecal samples were significantly less rich at day 2-5 compared to days 30 $(p=0.0023), 90(p=0.0019)$ and $120(p=0.0080)$ (Figure 3A, Table 2). A significantly lower level of Shannon diversity was also observed at day 2-5 compared to days $30(p=0.0435)$ and 90 ( $p=0.0199$ ) (Figure 3B, Table 2). However, beta diversity within maternal faecal samples was largely stable over time, apart from the day 2-5 sample which was significantly different from the day 30 sample $(p=0.0365)$ (Figure 3C, Table 3).

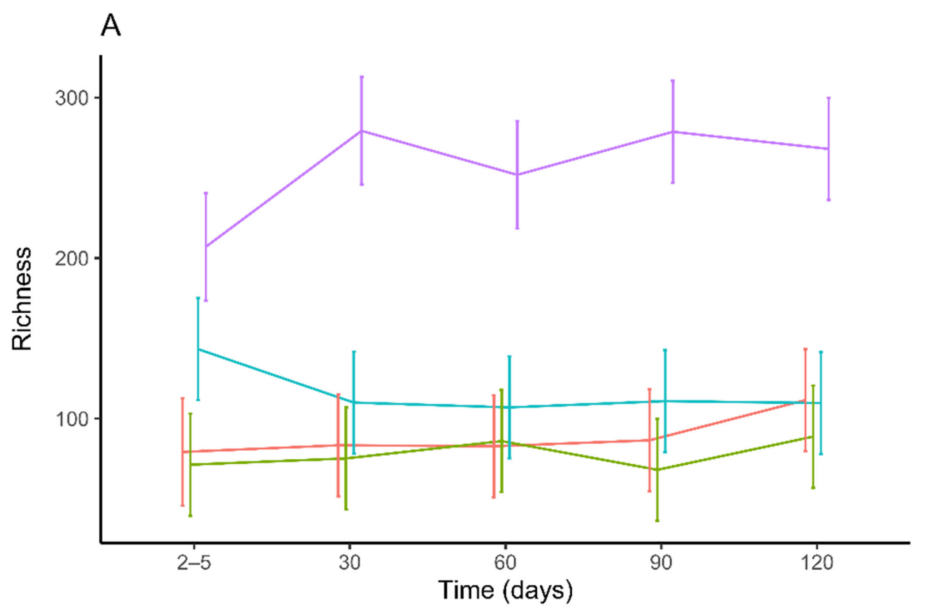

$$
\begin{aligned}
& \text { Sample } \\
& \text { - } \text { Human Milk } \\
& \text { — Infant Faecal } \\
& \text { — Infant Oral } \\
& \text { - Maternal Faecal }
\end{aligned}
$$

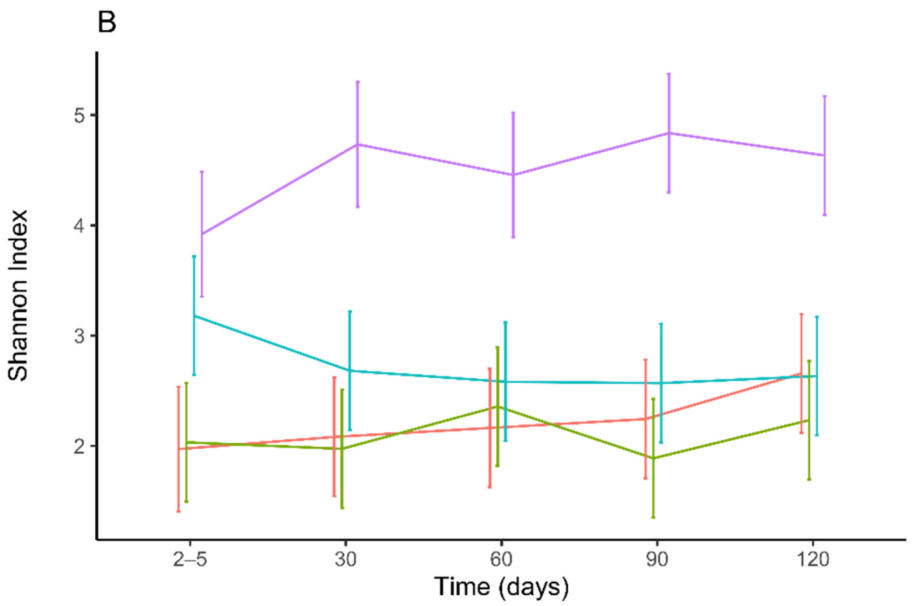

$$
\begin{aligned}
& \text { Sample } \\
& \text { - Human Milk } \\
& \text { — Infant Faecal } \\
& \text { — Infant Oral } \\
& \text { - Maternal Faecal }
\end{aligned}
$$

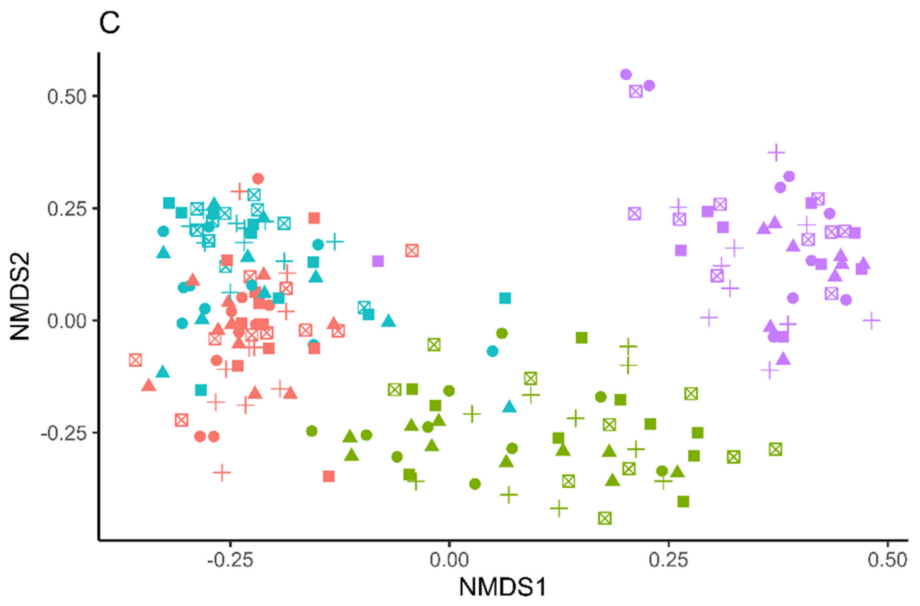

$$
\begin{array}{ll}
\text { Time (days) } \\
\text { - } & 2-5 \\
\text { - } & 30 \\
\text { - } & 60 \\
+ & 90 \\
\otimes & 120 \\
\text { Sample } \\
\text { - } \quad \text { Human Milk } \\
\text { - Infant Faecal } \\
\text { - Infant Oral } \\
\text { - } \text { Maternal Faecal }
\end{array}
$$

Figure 3. Alpha and beta diversities differ within and between sample types (human milk, infant faecal, infant oral and maternal faecal). (A) Richness (number of observed OTUs). (B) Shannon diversity. (C) NMDS plot of Bray Curtis dissimilarity distances. 
Table 2. Mean differences (MD), 95\% confidence intervals (CIs) and $p$-values for pairwise comparisons of alpha diversity measures (Richness and Shannon diversity) between time points, for each sample type.

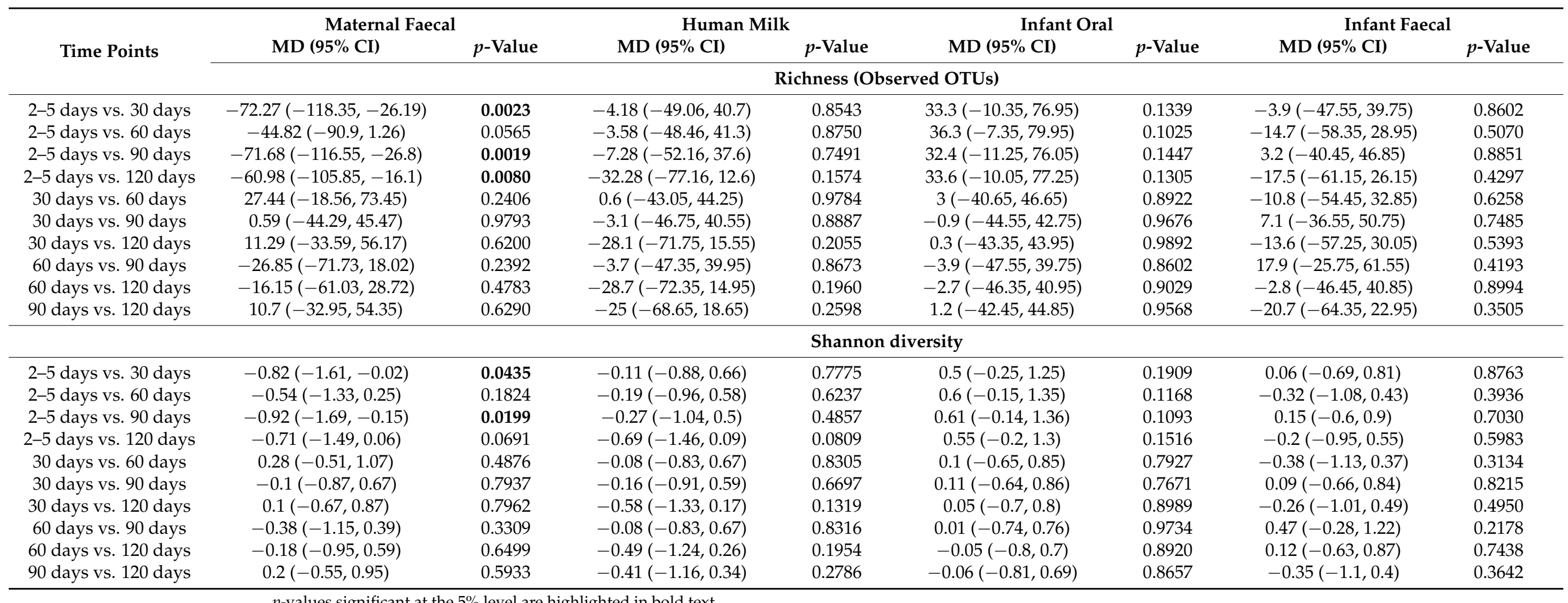


Table 3. PERMANOVA results assessing the beta diversity (Bray-Curtis dissimilarity) between time points, for each sample type.

\begin{tabular}{ccccc}
\hline \multirow{2}{*}{ Time Points } & Maternal Faecal & Human Milk & Infant Oral & Infant Faecal \\
\cline { 2 - 4 } & \multicolumn{4}{c}{ Bray-Curtis Dissimilarity } \\
\hline 2-5 days vs 30 days & $\mathbf{0 . 0 3 6 5}$ & 0.4227 & $\mathbf{0 . 0 0 2 9}$ & 0.3971 \\
2-5 days vs. 60 days & 0.1037 & 0.1354 & $\mathbf{0 . 0 2 1 1}$ & 0.4323 \\
2-5 days vs. 90 days & 0.3725 & 0.3969 & $<\mathbf{0 . 0 0 1}$ & 0.1590 \\
2-5 days vs. 120 days & 0.8534 & 0.2948 & $\mathbf{0 . 0 0 4 3}$ & 0.4046 \\
30 days vs. 60 days & 0.6497 & $\mathbf{0 . 0 2 8 9}$ & 0.6791 & 0.4193 \\
30 days vs. 90 days & 0.3358 & 0.0741 & $\mathbf{0 . 0 0 8 1}$ & 0.2825 \\
30 days vs. 120 days & 0.3102 & 0.0513 & $\mathbf{0 . 0 4 1 5}$ & 0.5278 \\
60 days vs. 90 days & 0.4660 & 0.6015 & 0.1343 & 0.2471 \\
60 days vs. 120 days & 0.5362 & 0.2185 & 0.6746 & 0.5739 \\
90 days vs. 120 days & 0.6205 & 0.5657 & 0.2726 & 0.3194 \\
\hline$p$-values significant at the 5\% level are highlighted in bold text.
\end{tabular}

\subsection{Temporal Development of Human Milk Bacterial Profiles}

Within HM samples, 10 OTUs were present at an average relative abundance of $>1 \%$ (Figure 1B) and collectively comprised $62.7-77.5 \%$ of the total bacterial profile. However, the relative abundance of these 10 OTUs changed over time. The most abundant OTU was OTU02 (Staphylococcus epidermidis) which dominated the HM taxa in the first month of life (32.2\% at day $2-5,41.2 \%$ at day 30 ) but fell in relative abundance at the later time points (13.9\% at day $60,20.7 \%$ at day 90 , and $13.5 \%$ at day 120$)$. The relative abundance of the other nine most abundant OTUs also changed over time.

The prevalence of seven OTUs (OTU02 (S. epidermidis), OTU05 (S. salivarius), OTU09 (Acinetobacter johnsonii), OTU14 (Veillonella nakazawae), OTU16 (Streptococcus lactarius), OTU26 (Dolosigranulum pigrum) and OTU28 (Staphylococcus hominis)) changed significantly over time (Figure 2B, Table A3).

Similar to the other sample types, we observed a high level of intra-individual and inter-individual variability in OTU composition over time (Figure A1B). Within and between mothers, the relative abundance of bacterial taxa changed across different time points.

Neither richness nor Shannon diversity differed over time within HM samples (Figure 3A,B, Table 2). However, beta diversity in day 30 samples differed significantly from day 60 samples $(p=0.0289)$ (Figure 3C, Table 3).

\subsection{Temporal Development of Infant Oral Bacterial Profiles}

Within infant oral samples, 11 OTUs were present at an average relative abundance of $>1 \%$ (Figure 1C) and collectively made up 57.5-69.6\% of the total bacterial profile. Variation in the relative abundance of these 11 OTUs was observed over time. For example, OTU01 (S. mitis), which dominated oral bacterial profiles, increased in relative abundance from day $2-5(10.4 \%)$ to day $30(20.0 \%), 60(26.3 \%), 90(43.1 \%)$ and $120(34.6 \%)$. Other early colonizers disappeared over time, such as OTU02 (S. epidermidis) which was present at day 2-5 (11.1\%) but quickly disappeared $(<1 \%$ relative abundance by day 30 and not present by day 120$)$.

Within individuals the prevalence of seven OTUs (OTU01 (S. mitis), OTU02 (S. epidermidis), OTU05 (S. salivarius), OTU11 (Haemophilus haemolyticus), OTU14 (V. nakazawae), OTU16 (S. lactarius) and OTU28 (S. hominis)) changed significantly over time (Figure 2C, Table A3).

There was a large degree of variability of OTUs within infant oral samples (Figure A1C). Additionally, when bacterial taxa composition was compared between infant oral samples, the relative abundance of dominating bacterial taxa changed (Figure A1C).

Neither richness nor Shannon diversity differed over time in infant oral samples (Figure 3A,B, Table 2). However, beta diversity was significantly different at day $2-5$ compared to all other time points (all $p<0.02$ ). Day 30 samples also differed significantly from day $90(p=0.0081)$ and $120(p=0.0415)$ samples (Figure 3C, Table 3$)$. 


\subsection{Temporal Development of Infant Faecal Bacterial Profiles}

Within infant faecal samples, 14 OTUs were present at an average relative abundance of $>1 \%$ (Figure 1D), and these collectively made up $69.4-77.9 \%$ of the total bacterial profile and varied in relative abundance over time. Overall, four OTUs (OTU03, OTU04, OTU10 and OTU24) mapping to Bifidobacterium species dominated the bacterial profile across all time points, increasing from day $2-5(38.0 \%)$ to $90(59.4 \%)$ and then decreasing at day $120(44.8 \%)$.

The presence/absence of five OTUs (OTU02 (S. epidermidis), OTU05 (S. salivarius), OTU14 (V. nakazawae), OTU20 (Bacteroides fragilis) and OTU24 (B. longum) changed significantly over time (Figure 2C, Table A3).

There was a large degree of intra-individual variation in bacterial composition over time (Figure A1D). Additionally, when bacterial taxa composition was compared between infants, the relative abundance of dominating bacterial taxa differed (Figure A1D).

Neither richness, Shannon diversity (Figure 3A,B, Table 2) nor beta diversity differed over time in infant faecal samples (all $p>0.05$ ) (Figure 3C, Table 3).

\subsection{Alpha and Beta Diversity between Sample Types}

Maternal faecal samples were the most rich (all $p<0.001$ ) and diverse (Shannon diversity, all $p<0.001$ ) at all time points compared to the other sample types (Figure 3A,B, Table 4), except for infant oral versus maternal faecal samples at day 2-5 ( $p=0.06)$. Infant oral samples were richer $(p<0.005)$ and more diverse in terms of Shannon diversity $(p<0.05)$ than HM and infant faecal samples at day $2-5$. Further, all sample types clustered separately from one another, demonstrating significant dissimilarity in their community structure (Bray-Curtis dissimilarity, all $p<0.02$ ), except for HM and infant oral samples at day $60(p=0.0563)$ (Figure 3C, Table 5).

\subsection{HMO Concentrations and Intakes over the First Four Months of Lactation}

Six HMOs (2'-fucosyllactose (2'FL), 3-fucosyllactose (3FL), difucosyllacto-N-tetrose (DFLNT), lacto-N-fucopentaose I (LNFP I), lacto-N-fucopentaose II (LNFP II) and lacto-Ntetrose (LNT)) made up the majority of HMO profiles (Figure 4). Concentrations of HMOs varied over time based on maternal secretor status. Among secretor mothers $(n=8)$, the concentrations of $2^{\prime} \mathrm{FL}, 6^{\prime}$-sialyllactose (6'SL), LNT, lacto-N-neotetraose (LNnT), LNFP I, lacto-N-fucopentaose III (LNFP III), sialyl-lacto-N-tetraose b (LSTb), sialyl-lacto-N-tetraose c (LSTc), DFLNT, lacto-N-hexaose (LHN), disialyllacto-N-tetraose (DSLNT), fucosyllactoN-hexaose (FLNH) and disialyllacto-N-hexaose (DSLNH) decreased significantly from day $2-5$ to 120 , while 3FL increased over time. Among non-secretor mothers $(n=2), 6^{\prime}$ SL, LNT, FLNH and DSLNH decreased, while 3FL significantly increased over time.

Additionally, daily intakes of HMOs differed over time in infants born to secretor and non-secretor mothers (Figure 5). Infants born to secretor mothers had higher intakes of 3FL at day 120 compared to day 30, while lower intakes of 2'FL, 6'SL LNT, LNFP I, LSTb, LSTc, DFLNT, LNH, DSLNT, FLNH and DSLNH were observed at day 120 compared to day 30. Infants born to non-secretor mothers had lower intakes of 3FL and higher intakes of $6^{\prime} \mathrm{SL}$, FLNH and DSLNH at day 30 compared to day 120. 
Table 4. Mean differences (MD), 95\% confidence intervals (CIs) and $p$-values for pairwise comparisons of alpha diversity measures (Richness and Shannon diversity) between sample types, at each time point.

\begin{tabular}{|c|c|c|c|c|c|c|c|c|c|c|}
\hline \multirow{2}{*}{$\begin{array}{l}\text { Sample } \\
\text { Types }\end{array}$} & \multicolumn{2}{|l|}{ Day 2-5 } & \multicolumn{2}{|l|}{ Day 30} & \multicolumn{2}{|l|}{ Day 60} & \multicolumn{2}{|l|}{ Day 90} & \multicolumn{2}{|l|}{ Day 120} \\
\hline & MD $(95 \%$ CI $)$ & $p$-Value & MD $(95 \% \mathrm{CI})$ & $p$-Value & MD $(95 \%$ CI $)$ & $p$-Value & MD $(95 \%$ CI $)$ & $p$-Value & MD $(95 \%$ CI $)$ & $p$-Value \\
\hline & \multicolumn{10}{|c|}{ Richness (Observed OTUs) } \\
\hline HM vs. IF & $7.92(-36.96,52.8)$ & 0.7280 & $8.2(-35.45,51.85)$ & 0.7112 & $-3.2(-46.85,40.45)$ & 0.8851 & $18.4(-25.25,62.05)$ & 0.4064 & $22.7(-20.95,66.35)$ & 0.3060 \\
\hline HM vs. IO & $-64.08(-108.96,-19.2)$ & 0.0054 & $-26.6(-70.25,17.05)$ & 0.2306 & $-24.2(-67.85,19.45)$ & 0.2753 & $-24.4(-68.05,19.25)$ & 0.2713 & $1.8(-41.85,45.45)$ & 0.9352 \\
\hline HM vs. MF & $-127.81(-173.89,-81.72)$ & $<0.0001$ & $-195.89(-240.77,-151.01)$ & $<0.0001$ & $-169.05(-213.92,-124.17)$ & $<0.0001$ & $-192.2(-235.85,-148.55)$ & $<0.0001$ & $-156.5(-200.15,-112.85)$ & $<0.0001$ \\
\hline IF vs. IO & $-72(-115.65,-28.35)$ & 0.0014 & $-34.8(-78.45,8.85)$ & 0.1174 & $-21(-64.65,22.65)$ & 0.3436 & $-42.8(-86.45,0.85)$ & 0.0546 & $-20.9(-64.55,22.75)$ & 0.3458 \\
\hline IF vs. MF & $-135.72(-180.6,-90.85)$ & $<0.0001$ & $-204.09(-248.97,-159.21)$ & $<0.0001$ & $-165.85(-210.72,-120.97)$ & $<0.0001$ & $-210.6(-254.25,-166.95)$ & $<0.0001$ & $-179.2(-222.85,-135.55)$ & $<0.0001$ \\
\hline \multirow[t]{2}{*}{ IO vs. MF } & $-63.72(-108.6,-18.85)$ & 0.0057 & $-169.29(-214.17,-124.41)$ & $<0.0001$ & $-144.85(-189.72,-99.97)$ & $<0.0001$ & $-167.8(-211.45,-124.15)$ & $<0.0001$ & $-158.3(-201.95,-114.65)$ & $<0.0001$ \\
\hline & \multicolumn{10}{|c|}{ Shannon diversity } \\
\hline HM vs. IF & $-0.06(-0.83,0.71)$ & 0.8746 & $0.11(-0.64,0.86)$ & 0.7765 & $-0.19(-0.94,0.56)$ & 0.6090 & $0.36(-0.39,1.11)$ & 0.3498 & $0.42(-0.33,1.17)$ & 0.2666 \\
\hline HM vs. MF & $-1.95(-2.74,-1.16)$ & $<0.0001$ & $-2.65(-3.42,-1.88)$ & $<0.0001$ & $-2.29(-3.06,-1.52)$ & $<0.0001$ & $-2.59(-3.34,-1.84)$ & $<0.0001$ & $-1.98(-2.73,-1.23)$ & $<0.0001$ \\
\hline IF vs. IO & $-1.15(-1.9,-0.4)$ & 0.0029 & $-0.71(-1.46,0.04)$ & 0.0640 & $-0.22(-0.97,0.53)$ & 0.5559 & $-0.68(-1.43,0.07)$ & 0.0747 & $-0.4(-1.15,0.35)$ & 0.2937 \\
\hline IF vs. MF & $-1.89(-2.66,-1.12)$ & $<0.0001$ & $-2.76(-3.53,-1.99)$ & $<0.0001$ & $-2.1(-2.87,-1.33)$ & $<0.0001$ & $-2.95(-3.7,-2.2)$ & $<0.0001$ & $-2.4(-3.15,-1.65)$ & $<0.0001$ \\
\hline IO vs. MF & $-0.74(-1.51,0.03)$ & 0.0603 & $-2.05(-2.82,-1.28)$ & $<0.0001$ & $-1.87(-2.65,-1.1)$ & $<0.0001$ & $-2.27(-3.02,-1.52)$ & $<0.0001$ & $-2(-2.75,-1.25)$ & $<0.0001$ \\
\hline
\end{tabular}

HM-human milk; IF-infant faecal; IO-infant oral; MF—-maternal faecal. $p$-values significant at the $5 \%$ level are highlighted in bold text. 

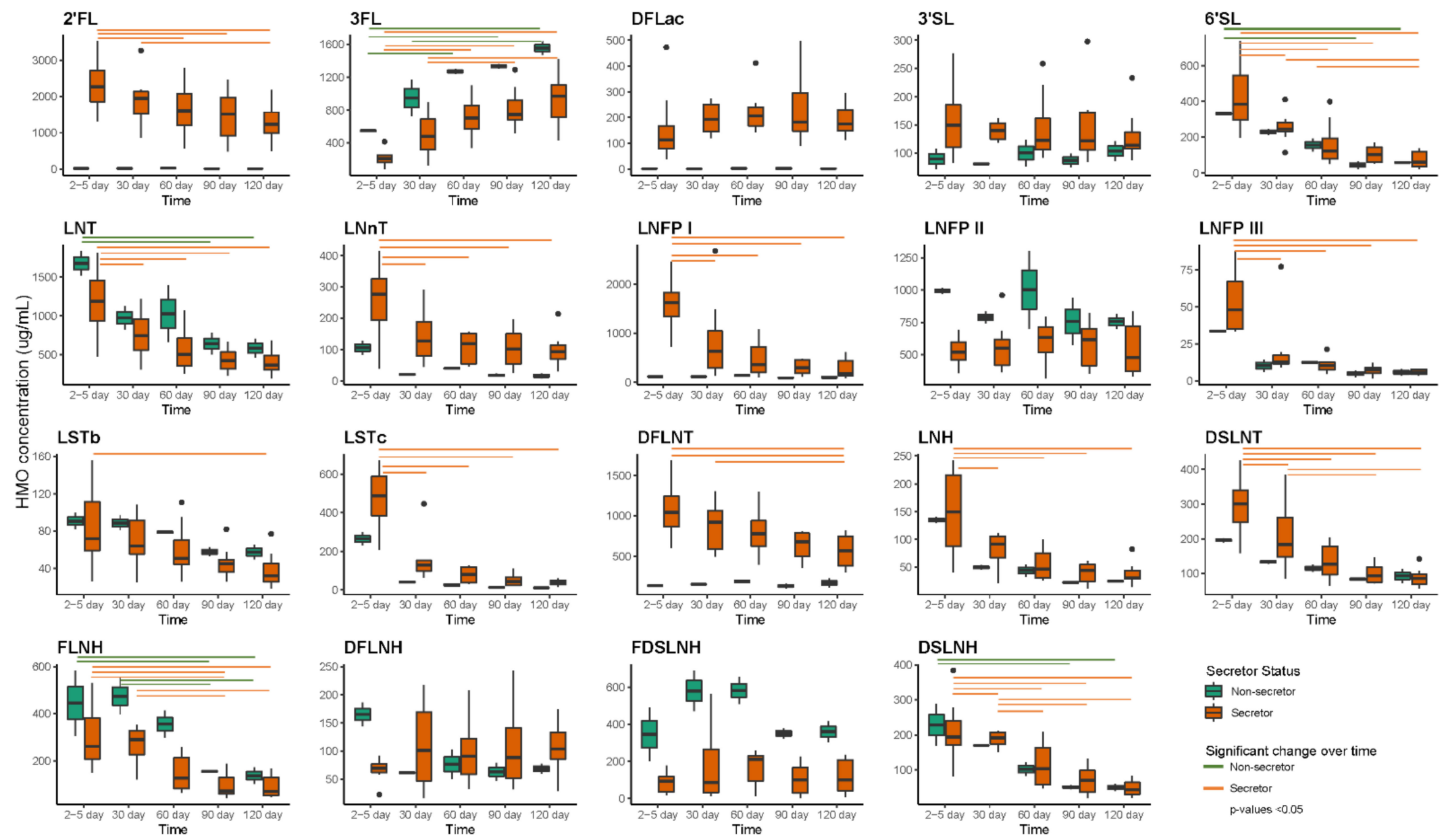

Figure 4. HMO concentrations over time within infants born to mothers with secretor $(n=8)$ and non-secretor $(n=2)$ status. Green and orange lines present significant difference within $\mathrm{HMO}$ concentration between time points. 2'FL-2'-fucosyllactose; 3'SL-3'-sialyllactose; 3FL-3-fucosyllactose; 6'SL-6'-sialyllactose; DFLacdifucosyllactose; DFLNH—difucosyllacto-N-hexaose; DFLNT—difucosyllacto-N-tetrose; DSLNH—disialyllacto-N-hexaose; DSLNT-disialyllacto-N-tetraose; FDSLNH-fucodisialyllacto-N-hexaose; FLNH—fucosyllacto-N-hexaose; LNFP I-lacto-N-fucopentaose; LNFP II-lacto-N-fucopentaose II; LNFP III-lacto-Nfucopentaose; LNH-lacto-N-hexaose; LNnT-lacto-N-neotetraose; LNT-lacto-N-tetrose; LSTb—sialyl-lacto-N-tetraose b; LSTc—sialyl-lacto-N-tetraose c. 

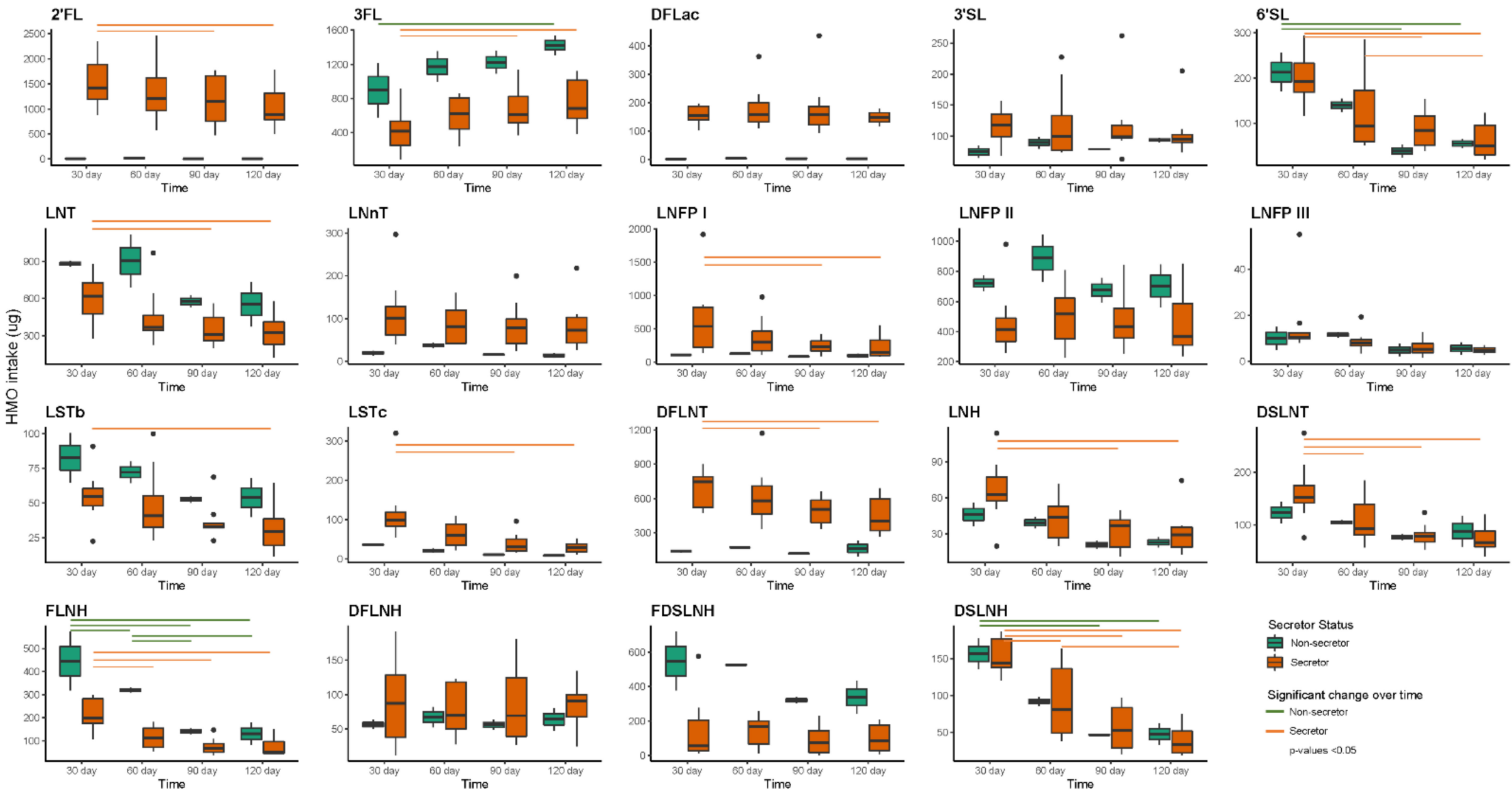

Secretor Status

官 Non-secre

Secretor

Significant change over time

- Non:secretor

- secretor

Figure 5. Twenty-four-hour intakes of the HMOs within infants born to mothers with secretor $(n=8)$ and non-secretor $(n=2)$ status. Green and orange lines present significant difference within HMO intakes between time points. $2^{\prime} \mathrm{FL}-2^{\prime}$-fucosyllactose; $3^{\prime} \mathrm{SL}-3^{\prime}$-sialyllactose; $3 \mathrm{FL}-3$-fucosyllactose; $6^{\prime} \mathrm{SL}-6^{\prime}$-sialyllactose; DFLacdifucosyllactose; DFLNH—difucosyllacto-N-hexaose; DFLNT—difucosyllacto-N-tetrose; DSLNH—disialyllacto-N-hexaose; DSLNT—disialyllacto-N-tetraose; FDSLNH—fucodisialyllacto-N-hexaose; FLNH—fucosyllacto-N-hexaose; LNFP I-lacto-N-fucopentaose; LNFP II-lacto-N-fucopentaose II; LNFP III-lacto-Nfucopentaose; LNH-lacto-N-hexaose; LNnT-lacto-N-neotetraose; LNT_lacto-N-tetrose; LSTb—sialyl-lacto-N-tetraose b; LSTc-sialyl-lacto-N-tetraose c. 
Table 5. PERMANOVA results assessing the beta diversity (Bray-Curtis dissimilarity) between sample types at each time point.

\begin{tabular}{|c|c|c|c|c|c|}
\hline \multirow{2}{*}{ Sample Types } & Day 2-5 & Day 30 & Day 60 & Day 90 & Day 120 \\
\hline & \multicolumn{5}{|c|}{ Bray-Curtis Dissimilarity } \\
\hline Human milk vs. Infant faecal & $<0.0001$ & $<0.0001$ & $<0.0001$ & $<0.0001$ & $<0.0001$ \\
\hline Human milk vs. Infant oral & 0.0218 & 0.0001 & 0.0563 & 0.0004 & $<0.0001$ \\
\hline Human milk vs. Maternal faecal & 0.0004 & $<0.0001$ & 0.0002 & $<0.0001$ & $<0.0001$ \\
\hline Infant faecal vs. Infant oral & $<0.0001$ & 0.0006 & $<0.0001$ & $<0.0001$ & $<0.0001$ \\
\hline Infant faecal vs. Maternal faecal & 0.0002 & $<0.0001$ & $<0.0001$ & $<0.0001$ & $<0.0001$ \\
\hline Infant oral vs. Maternal faecal & $<0.0001$ & $<0.0001$ & 0.0069 & $<0.0001$ & $<0.0001$ \\
\hline
\end{tabular}

\subsection{Associations between HMO Intake and Infant Oral Microbiota}

Intakes of individual HMOs showed both positive and negative associations with different categories of relative abundance and different bacterial OTUs in the infant oral cavity at different time points (Table A4).

At day 30, higher intakes of seven individual HMOs (LNnT, LNFP I, LNFP III, LSTc, LNH, DSLNT, FLNH) were positively associated with different relative abundances of four bacterial OTUs (OTU05 (S. salivarius), OTU11 (H. haemolyticus), OTU18 (Veillonella sp. oral clone ASCB03), and OTU32 (Haemophilus parainfluenzae)). Opposingly, higher intakes of DSLNT was associated with the absence of OTU18 (Veillonella sp. oral clone ASCB03) than when this OTU was present.

At day 60, higher intakes of eight individual HMOs (3FL, difucosyllactose (DFLac), DFLNT, DSLNT, LNnT, LNFP I, LNFP II, LNFP III, LSTb and LSTc) were positively associated with different relative abundances of five bacterial OTUs (OTU01 (S. mitis), OTU03 (B. longum subsp. infantis), OTU14 (V. nakazawae), OTU18 (Veillonella sp. oral clone ASCB03), and OTU32 (H. parainfluenzae)). Higher intakes of 3FL and LNFP II were associated with the absence of OTU02 (S. epidermidis).

At day 90, higher intakes of five individual HMOs ( $3^{\prime}$ SL ( $3^{\prime}$-sialyllactose), DFLac, LNFP I, LSTC and DSLNT) were positively associated with different relative abundances of three bacterial OTUs (OTU01 (S. mitis), OTU18 (Veillonella sp. oral clone ASCB03), and OTU32 (H. parainfluenzae)). Higher intakes of six HMOs (6'SL, LNT, LNFP II, LSTb, DSLNH and DSLNT) were associated with the absence of four bacterial OTUs (OTU05 (S. salivarius), OTU07 (G. haemolysans), OTU19 (R. mucilaginosa), and OTU22 (Bergeyella sp.).

At day 120, higher intakes of seven individual HMOs (2'FL, $3^{\prime} \mathrm{SL}, 6^{\prime} \mathrm{SL}$, LNFP I, LNH, fucodisialyllacto-N-hexaose (FDSLNH), and DSLNH) were positively associated with different relative abundances of four bacterial OTUs (OTU03 (B. longum subsp. infantis), OTU05 (S. salivarius), OTU18 (Veillonella sp. oral clone ASCB03), and OTU32 (H. parainfluenzae). Higher intakes of four HMOs ( $2^{\prime} \mathrm{FL}, 6^{\prime} \mathrm{SL}$, LNFP I and LNH) were associated with absence of three bacterial OTUs (OTU01 (S. mitis), OTU03 (B. longum subsp. infantis), and OTU18 (Veillonella sp. oral clone ASCB03)).

\subsection{Associations between HMO Intake and Infant Faecal Microbiota}

Similar to infant oral samples, individual HMO intakes showed both positive and negative associations with different categories of relative abundance and different bacterial taxa at different time points in infant faecal samples (Table A5).

At day 30, overall, higher intakes of 12 HMOs (3FL, 3'SL, LNnT, LNFP I, LNFP II, LNFP III, LSTb, LSTc, DSLNT, FLNH, difucosyllacto-N-hexaose (DFLNH) and DSLNH) were positively associated with different relative abundances of eight bacterial OTUs (OTU03 (B. longum subsp. infantis), OTU05 (S. salivarius), OTU10 (B. pseudocatenulatum), OTU13 (Raoultella ornithinolytica), OTU17 (Klebsiella pneumoniae), OTU20 (B. fragilis), OTU24 (B. longum), and OTU25 (Parabacteroides distasonis)). Opposingly, higher intakes of 2'FL, 
DFLac and DFLNT were associated with the absence of OTU04 (B. breve) and a higher intake of 3'SL was associated with the absence of OTU03 (B. longum subsp. infantis).

At day 60, higher intakes of LNT, LNnT, LNFP I, LNFP II, LNFP III, LSTb, DFLNT, FLNH, LNH, DFLNH were positively associated with different relative abundance of six bacterial OTUs (OTU04 (B. breve), OTU06 (E. coli), OTU10 (B. pseudocatenulatum), OTU17 (K. pneumoniae), OTU25 (P. distasonis), and OTU29 (Enterococcus faecalis)). A higher intake of 3FL was associated with the absence of OTU05 (S. salivarius) and a higher intake of FLNH was associated with absence of OTU20 (B. fragilis).

At day 90, higher intakes of LNnT, LNFP III, LSTc and FDSLNH were positively associated with different relative abundances of four bacterial OTUs (OTU03 (B. longum subsp. infantis), OTU13 (R. ornithinolytica), OTU25 (P. distasonis), and OTU29 (E. faecalis)). Higher intakes of $6^{\prime}$ SL and DFLNT were associated with absence of OTU24 (B. longum) and a higher intake of FLNH was associated with absence of OTU04 (B. breve).

At day 120, higher intakes of $2^{\prime} \mathrm{FL}, 3^{\prime} \mathrm{SL}$, LNT, LNFP I, LNFP II, DFLNT LNH, FLNH and FDSLNH were positively associated with different relative abundances of six bacterial OTUs (OTU05 (S. salivarius), OTU06 (E. coli), OTU20 (B. fragilis), OTU24 (B. longum), OTU25 (P. distasonis), and OTU29 (E. faecalis)). In contrast, higher intake of 3FL was associated with absence of OTU05 (S. salivarius), DFLac with OTU24 (B. longum) and OTU25 (P. distasonis), DFLNH with OTU14 (V. nakazawae), and LNFP II with OTU05 (S. salivarius).

\section{Discussion}

This study is one of the few to characterize the temporal development of four interrelated human microbial niches from mother-infant dyads during the exclusive breastfeeding period. As expected, the bacterial profile of each microbiome was unique and changed over time with HMO intakes associating with changes in the infant oral and faecal microbiomes.

During pregnancy the maternal gut microbiota is remodeled with a decrease in bacterial richness during the third trimester and one-month postpartum [38]. Aberrant postpartum microbiota may have negative implications for both mother and infant health $[58,59]$. Despite this, temporal development of the maternal gut microbiome postpartum has not been well characterized. We observed the most abundant bacterial taxa changing across different time points, with Dialister invisus and Bacilli_c;RF39_o;RF39_fa;RF39_ge dominating at day $2-5, P$. vulgatus at months one, two and four, and P. vulgatus and E. coli dominating three-month bacterial profiles. Interestingly, our results differ to those from studies that used strain-level metagenomic profiling at different time points (shortly after delivery within $24 \mathrm{~h} \mathrm{[13]} \mathrm{and} \mathrm{at} \mathrm{three} \mathrm{months} \mathrm{[34]),} \mathrm{with} \mathrm{the} \mathrm{exception} \mathrm{of} \mathrm{Faecalibacterium}$ prausnitzii. The difference could be due to geographical and/or dietary variances, as these metagenomic analyses [13,34] were based on Italian mothers and diet [60], lifestyle differences [61], and methodological differences between 16S ribosomal RNA (rRNA) and shotgun sequencing have been shown to be related to the gut microbiome [62].

Significant changes in the prevalence of certain bacterial taxa as well as increases in bacterial richness and Shannon diversity and changes in beta diversity within the maternal microbiota were observed between day $2-5$ and one month postpartum. These findings are in stark contrast to previous studies, which have not detected changes, instead reporting stability of diversity and relative distribution of bacterial communities over time $[24,39]$. The differences observed in this study between first-week and one-month maternal faecal microbiota may be due to a number of factors, including change in maternal diet [63-65] as well as the hormonal changes associated with both labour and lactation, such as the dramatic decrease in progesterone and increase in oxytocin and estrogen [66,67]. Additionally, pregnancy and labour are associated with stress, which has been associated with delayed onset of lactation [68] and an increase of intestinal permeability [69], potentially allowing bacteria to travel across the intestinal mucosa. However, we did not analyse pre-pregnancy samples, so it is difficult to know whether the day $2-5$ samples are different from the pregnancy sample or not. Future work is needed to prospectively follow women 
from preconception through pregnancy and into the postpartum period with the addition of documenting maternal measures of metabolic, hormonal, and immune changes together, to assess the impact of pregnancy, birth, and lactation on the maternal gut microbiome.

While previous efforts have been made to characterize the HM bacterial profile using metagenomic $[35,37,70,71]$ and $16 \mathrm{~S}$ rRNA gene sequencing $[14,18,41-43,47]$ methods, only one study has aimed to characterise HM temporal development during the first six months of breastfeeding. William et al. analysed HM bacterial profiles using partial 16S rRNA gene sequencing, at nine different time points, from day two until six months postpartum (52). They reported Streptococcus sp. and Staphylococcus sp. were dominant and relatively constant over time with less-abundant genera such as Veillonella sp., Propionibacterium sp., Prevotella sp. and Granulicatella sp. increasing over time. Our study builds upon this by using full-length $16 \mathrm{~S}$ rRNA gene sequencing for improved taxonomic resolution. We found 10 bacterial OTUs mapping to Staphylococcus epidermidis, Streptococcus salivarius, Streptococcus mitis, Gemella haemolysans, Streptococcus agalactiae, Cutibacterium acnes, Acinetobacter johnsonii, Moraxella osloensis, Streptococcus lactarius and Streptococcus anginosus dominated HM bacterial profiles and that the most abundant OTUs changed over time.

Previous studies documenting differences in HM bacteria with lactation stage have mainly focused on the transition from colostrum to mature milk [14,19,44,45]. By extending the time of sample collection, we found that some abundant species, such as S. epidermidis, were more prevalent in the first month postpartum compared to two and four months. S. epidermidis is a ubiquitous commensal of the skin and mucosal environments [72,73] and has emerged as the predominant pathogen of sepsis in preterm infants [74]. The conversion of $S$. epidermidis from commensal skin inhabitant to a virulent pathogen may be due to disruption of the skin epithelial barrier or through selective pressure due to extensive use of antibiotics in preterm infants [75]. However, in healthy term infants, S. epidermidis primarily plays a commensal role by inhibiting virulent pathogens and educating and stimulating the innate immune system [75]. Indeed, a recent study has shown that the gut and skin of term neonates were colonized with strains of S. epidermidis genetically similar to those present in HM [76], supporting a role for HM bacteria in infant microbiome colonization. Our data suggest that bacteria in HM are temporally dynamic, meaning that infants are exposed to a differing combination of bacteria across time. While the composition of the HM microbiome differed over time, we did not observe differences in richness and Shannon diversity over time, similar to a previous study [77] but in contrast to another [44]. This suggests that geographic, genetic, and dietary factors may influence bacterial community structure in milk.

The oral cavity serves as an initial entry point for colonization of the oral and gut microbiota [78,79], and thus, the oral microbiota influences infant health [79] with early life dysbiosis being related to conditions such as dental caries, periodontitis and oral mucosal diseases [80,81]. As such, characterisation of oral temporal development during the exclusive breastfeeding period is important to identify early factors that may disturb optimal colonisation. We found that 11 bacterial OTUs (S. epidermidis, S. salivarius, S. mitis, G. haemolysans, V. nakazawae, B. longum subsp. infantis, H. haemolyticus, R. mucilaginosa, Bergeyella sp., H. parainfluenzae and Veillonella sp. oral clone ASCB03) dominated the infant oral microbiota. Our findings are in agreement with a US study that found a core microbial community of the S. mitis group, R. mucilaginosa, S. salivarius, and G. haemolysans in the first three months of life [27]. However, we did not observe the previously reported Veillonella parvula group and Veillonella HB016 [27]. The differences between studies could be due to host genetic variations, ethnicity and geographical location, as these have been shown to influence the oral microbiota [82-84].

We also found that the relative abundance and prevalence of these OTUs in the infant oral cavity changed over time. S. mitis was one of the most abundant and ubiquitous OTUs, whose relative abundance more than doubled from day $2-5$ to all other time points. S. mitis is major oral organism and is likely to modulate oral colonization of other bacterial species, as demonstrated in previous studies $[85,86]$. Additionally, the infant oral cavity contains 
high levels of the metabolites xanthine and hypoxanthine [87]. An in vitro study that added these metabolites to HM showed production of hydrogen peroxide. If this translates to an in vivo setting, this may in turn inhibit the growth of opportunistic pathogens such as Staphylococcus aureus, Pseudomonas aeruginosa and Salmonella spp. $[86,88]$. This could help explain our observation of S. epidermidis being dominant in the infant oral microbiota after birth, but reducing to $<1 \%$ over the first month and then being absent at four months. A recent in vitro study by Sweeney et al. reported immediate inhibition of S. epidermidis when a saliva-HM mixture was supplemented with hypoxanthine and xanthine [89]. Future studies investigating the temporal development of oral microbiota should also consider analyzing oral bacterial metabolites as they may influence oral colonization.

Conflicting data exist with respect to the diversity of the infant oral microbiome. For example, we found that neither richness nor Shannon diversity in infant oral samples differed over the first four months of life. Similarly, Hurley et al. reported that Shannon diversity remained stable with increasing age [90], while in contrast, Sulyanto et al. reported Shannon diversity increased over time [27]. This result might be confounded by the feeding methods used, as three infants were solely breastfed, one solely formula-fed, and five were mix-fed [27]. Previous longitudinal studies have shown bacterial richness increases and the compositional profile changes with age between three months and seven years [31,91-93]; however, this is also likely to be due to consumption of food and fluids other than HM [27,94]. Our beta diversity analysis showed diversity significantly increased from day 2-5 to one, two, three and four months, highlighting the continuous development and maturation of the oral microbiota in infancy.

Infant oral microbiota are important for oral health [79] and differences in the oral microbiota between breastfed and formula-fed infants have been reported [91,95,96], however, little is known about how HMOs impact the oral microbiota. A recent in vitro study assessed the effect of $2^{\prime} \mathrm{FL}$ and galacto-oligosaccharides on the growth and adhesion characteristics of the caries-associated oral pathogen Streptococcus mutans and reported 2'FL both limits growth and inhibits adhesion of $S$. mutans to saliva-coated hydroxyapatite [97]. However, there is an absence of information on how daily intakes of HMOs influence infant oral microbiota. The current study is the first to provide an insight into associations between daily intakes of various HMOs and infant oral bacteria; however, these associations differed over time (Table A4). Additionally, we observed several individual HMO intakes supported the growth of infant oral bacteria such as Veillonella spp., S. mitis and S. salivarius, which are common oral cavity inhabitants [98-100]. Furthermore, we observed intakes of 3FL and LNFP II were negatively associated with abundance of S. epidermidis in the infant oral cavity. S. epidermidis is one of the most abundant colonizers of skin and mucosal surfaces [101] and is associated with dental caries [102]. Therefore, the results from the current study may suggest a protective role of HMOs in the infant oral cavity as well as involvement of HMOs in establishment of the infant oral microbiota. However, results from the current study need to be confirmed in larger longitudinal cohorts.

The early life infant gut microbiota is often regarded as having high plasticity due to its low diversity and rapid development [103]; however, neither have been extensively studied during the exclusive breastfeeding period. Carrothers et al. found Bacteroides sp., Faecalibacterium sp., Lachnospiraceae incertae sedis sp. and Prevotella sp. dominated the infant faecal microbiota from day two to six months, while Bifidobacterium sp. only made up a small proportion [39]. Contrarily, we observed that Bifidobacterium species including B. longum subsp. infantis, B. breve, B. pseudocatenulatum and B. longum dominated the bacterial profile over the first four months of life. Previous studies using strain-level analyses during the first year of life have reported that the breastfed infant gut is dominated by Bifidobacterium species, including B. longum subsp. longum, B. breve, B. bifidum, B. longum subsp. infantis, B. adolescentis and B. pseudocatenulatum $[9,34,35,37,104]$. In comparison, formula-fed infants have been found to have a more diverse bacterial community and higher abundance of Clostridium difficile, Granulicatella adiacens, Citrobacter spp., Enterobacter cloacae and Bilophila wadsworthia [105]. The high levels of Bifidobacterium spp. in breastfed infants are likely due 
to HMOs, which promote the growth of this genera [106-109]. Bifidobacterium spp. are early colonisers of the infant gut, and producers of aromatic lactic acids, such as indole lactic acid, which modulate intestinal immune responses via their interaction with the aryl hydrocarbon receptor $[110,111]$. This emphasizes the role of Bifidobacterial priming and programming of immune functionality in early life. We also observed no change in richness, Shannon diversity and beta diversity over time in infant faecal samples. However, others have observed changes [112,113], that may be associated with the introduction of solids and/or cessation of breastfeeding, promoting the survival and proliferation of varied types of microbial species $[105,114]$. A greater understanding of the temporal development of the gut during early life may identify signatures that are less favourable to positive health outcomes and thus enable development of potential interventions to improve short- and long-term health.

One of the potent factors shaping the temporal development of exclusively breastfed infant gut bacterial profiles are HMOs, the third most abundant component of HM, that acts as a prebiotic for bacterial colonisation in the infant gut amongst other functions [115-117]. It has been reported that most HMOs are non-digestible and reach the colon undigested [118,119], where they serve as a carbon source for bacterial fermentation [120,121] and are involved in host-microbe interactions [122]. Substantial evidence exists showing associations between $\mathrm{HMO}$ concentrations and infant gut bacteria including Bifidobacterium sp., Lactobacillus sp., Bacteroides sp., Veillonella sp., Enterococcus sp. and Streptococcus sp. $[53,55,123,124]$. However, no study to date has evaluated the impact of HMO daily intakes on infant gut microbiota. A novel finding of this study is a detection of both positive and negative associations between various individual HMO daily intakes and infant gut bacteria (B. longum subsp. infantis, S. salivarius, E. coli, B. pseudocatenulatum, R. ornithinolytica, K. pneumoniae, B. fragilis, B. longum, P. distasonis, and E. faecalis). However, these associations differed over time. For example, at one-month intakes of $2^{\prime}$ FL, DFLac and DFLNT were negatively associated B. breve, while intake of DFLNH was positively associated at two months, and intake of FLNH negatively associated at three months. The differences between different time points could be a result of concentrations of HMOs significantly changing over time [125], thereby influencing HMO daily intakes. In addition to HMOs, there are likely other mechanisms influencing microbial community structure. For example, the presence of other components in HM such as total protein [53], lysozyme [126], secretory immunoglobulins [127] and other endogenous factors have been shown to influence the infant gut microbiota.

One possible explanation for associations of infant gut Bifidobacterium spp. and Bacteroides spp. with certain HMO intakes in our study could be due to their genetic material. Marcobal et al. analyzed the genomes of 16 bacterial strains of gut microbiota and reported Bacteroides fragilis, Bacteroides vulgatus, Bifidobacterium infantis and Bifidobacterium longum have different genes coding for production of enzymes such as $\alpha$-galactosidase, $\beta$ $\mathrm{N}$-acetylgalactosaminidase, $\beta$-hexosaminidase, $\alpha$-L-fucosidase, sialidase, $\beta$-galactosidase, and $\alpha 1,2$-L-fucosidase for glycoside hydrolases of HMOs [52]. However, the reason for HMOs associations with other gut bacteria is unclear. It is plausible that other gut bacteria have been directly utilizing the HMOs or they were benefitting from cross-feeding of HMOs fermented by Bifidobacterium spp. and Bacteroides spp [108,128]. Moreover, these bacteria are able to convert HMOs into short-chain fatty acids such as lactate, acetate, propionate and butyrate [129], which serve as nutrients for cross-feeding between gut bacteria [130]. The advantage of Bifidobacterium spp. and Bacteroides spp. of HMO utilization may promote diversity and dominance of these bacteria during early life while down regulating the colonization of other taxa. Additionally, HMOs act as receptor decoys and prevent the binding of Clostridia, Campylobacter, and the stable toxin of entero-toxigenic E. coli to their target host cell receptors [131], thus limiting their colonization in the infant gut. However, future studies are required to analyze the metagenome of gut microbiota to identify if other gut bacteria contain genes that allow the production of enzymes which could help to utilize HMOs. 
HMO composition varies between mothers and is dependent on maternal genetics [132,133], with many HMOs significantly decreasing in concentration over the first two years of lactation $[125,134]$. Indeed, we found that the concentrations of 13 HMOs in secretor mothers and four HMOs in non-secretor mothers decreased across the first four months, except for 3FL, which increased in concentration in both secretor and non-secretor mothers as lactation progressed, which is consistent with reports by Plows et al. [125]. Additionally, a novel aspect of this study was documenting changes in the daily intakes of HMOs over the first four months of life in infants born to secretor and non-secretor mothers. However, calculation of daily intakes gave differing results, with 11 individual $\mathrm{HMO}$ intakes of infants born to secretor mothers and three HMO intakes of infants from non-secretor mothers decreasing from month one to four. The daily intake of 3FL was however significantly higher across the same period for infants of both secretor and nonsecretor mothers. To date only one study has measured HMO intakes and compared intake differences between infants born to mothers with normal weight, over-weight and obesity status [135]. They reported infants born to mothers with obesity had lower intakes of LNH, FLNH, DFLNH, DFLNT, and DSLNH compared to infants born to mothers with normal weight and over-weight status. Although these results are not comparable to our study, they provide evidence of maternal influence on infant $\mathrm{HMO}$ intakes.

The strengths of this study include the exclusivity of breastfeeding, full-length $16 \mathrm{~S}$ rRNA gene sequencing and measurement of daily HMO intakes. The limitations include low participant numbers $(n=10)$ which did not allow for stratification based on maternal secretor status for the analysis of associations between HMO intakes and the infant microbiomes, therefore results should be interpreted cautiously. Despite achieving high sequencing read numbers, sequencing coverage for maternal faecal samples was greatly reduced compared to coverage values for human milk, infant oral and infant faecal samples. This indicates that microbial diversity in these samples is higher than reported here and is likely due to sequencing low (human milk, infant oral and infant faecal) and high (maternal faecal) biomass samples together. Future studies should consider separately preparing low and high biomass sample libraries for sequencing in order to ensure appropriate sequencing coverage across all samples. We do note though, that although this is a limitation of the current study, the samples relevant to the primary aim, describing longitudinal development of the infant gut microbiome, all have sufficient sequencing coverage. Further, our population consisted of vaginally delivered term, healthy, exclusively breastfed infants from Caucasian mothers of high social-economic status living in Australia; therefore, the results may not be transferable to other populations. Nonetheless, this study has yielded important findings that warrant validation in larger longitudinal cohorts with extensive sampling.

\section{Materials and Methods}

\subsection{Study Design}

Participants were recruited during the third trimester of pregnancy ( $>30$ weeks gestation) to participate in the BLOSOM (Breastfeeding Longitudinal Observational Study of Mothers and kids) study, as previously described [136]. In this sub-study, 10 mother-infant pairs were chosen based on the following additional criteria; healthy women with no major pregnancy complications, vaginal birth, term infant, exclusively breastfeeding, no maternal smoking, no maternal or infant antibiotic use during labour or in the first four months postpartum, no maternal nipple pain, no infant pacifier use in the first 5 days of life, and no solid introduction before four months of age. The study was approved by the Human Research Ethics Committee at The University of Western Australia (RA/4/20/4023); all participants provided informed written consent to participate.

\subsection{Sample and Data Collection}

Mothers answered a background questionnaire at the time of recruitment and collected their own and their infant's samples during the study at five time points: 2-5, 30, 60, 90 and 120 days postpartum. 
Mothers selected one breast from which to donate HM samples throughout the study and were asked not to breastfeed or express milk from the breast for at least two hours prior to sample collection. Mothers washed their hands thoroughly with soap and water and wore gloves during sample collection. The nipple and areola of the expressing breast were cleaned with prep pads (70\% isopropyl alcohol and $2 \%$ chlorhexidine digluconate, Reynard Health Supplies, Artarmon, NSW, Australia), followed by rinsing with sterile saline solution (Livingstone, Mascot, NSW, Australia) and drying with sterile gauze swabs (Livingstone, Mascot, NSW, Australia). Up to $20 \mathrm{~mL}$ (otherwise as much as possible) of HM was expressed directly into sterile tubes using hand-expression, as previously described [137].

Maternal faecal samples were collected from toilet paper using an E-swab (Becton, Dickinson and Company, Franklin Lakes, NJ, USA). Infant faecal samples were collected from diapers within 1-2 h post-bowel movement using an E-swab and avoiding any urine. For infant oral samples, the E-swab was firmly rubbed up and down and in a circular motion against the inside of the cheek 10 times. Using the same E-swab, the process was repeated on the other side. E-swabs were carefully removed from the mouth without touching the lips or other surfaces and were preserved in $1 \mathrm{~mL}$ liquid Amies medium.

All samples were stored in the refrigerator at the participant's home for up to $18 \mathrm{~h}$ before being collected and transported on ice to the laboratory, where they were immediately aliquoted into sterile tubes (Sarstedt, Numbrecht, Germany) and stored at $-80^{\circ} \mathrm{C}$ until further analysis. All samples collected by E-swab were eluted into the collection media by vortexing for $5 \mathrm{~s}$ prior to aliquoting.

\subsection{Human Milk Oligosaccharides Analysis}

$100 \mu \mathrm{L}$ of HM aliquots from each participant $(n=10)$ per time point were sent on dry ice to the Bode Lab at the University of California, (San Diego, CA, USA). The concentration and composition of HMOs in HM samples was analyzed by HPLC after labelling with the fluorescent tag 2-aminobenzamide as described previously [138]. The following $19 \mathrm{HMOs}$ were identified and quantified: 2'FL, 3FL, 3'SL, 6'FL, DFLac, DFLNH, DFLNT, DSLNH, DSLNT, FDSLNH, FLNH, LNFP I, LNFP II, LNFP III, LNH, LNnT, LNT, LSTb and LSTc. Maternal secretor status was identified based on the presence or near-absence of $2^{\prime} \mathrm{FL}$ in HM.

\subsection{4-h Milk Intake}

Infant 24-h milk intake was measured at the three month time point by mothers in their homes using the 24-h milk profile protocol as described previously [139]. Briefly, mothers weighed their infant before and after each feed on electronic scales $( \pm 2.0 \mathrm{~g}$; Electronic Baby Weigh Scale, Medela Inc., McHenry, IL, USA). HM intake (g) was calculated by subtracting the weight of the infant before the feed from the weight after the feed. Three months 24-h milk intakes were considered representative of intakes during the exclusive breastfeeding period as there is no significant variation in HM intake from one to six months within infants [140].

\subsection{Daily Intakes of HMOs}

HMO daily intakes $(\mu \mathrm{g})$ were determined as the concentration of HMOs $(\mu \mathrm{g} / \mathrm{mL})$ multiplied by 24-h milk intake (grams).

\subsection{DNA Extraction and Quantification}

One $\mathrm{mL}$ aliquots of $\mathrm{HM}$ were centrifuged at $40,000 \times \mathrm{g}$ for $5 \mathrm{~min}$ at $4{ }^{\circ} \mathrm{C}$. The supernatant and lipid fraction were discarded. Maternal faecal, infant faecal, and infant oral samples were centrifuged at $40,000 \times g$ for $5 \mathrm{~min}$ at $4{ }^{\circ} \mathrm{C}$ and the supernatant was discarded. For all sample types, DNA was extracted from the cell pellet using the QIAGEN MagAttract Microbial DNA Isolation Kit (QIAGEN, Hilden, Germany) on the Kingfisher Flex platform, following the manufacturer's instructions. Two negative extraction controls each consisting 
of $1 \mathrm{~mL}$ sterile nuclease-free water (Integrated DNA Technologies, Coralville, IA, USA) were included at the centre of each 96-well extraction plate.

Total DNA yield was assessed using the Qubit ${ }^{\circledR}$ dsDNA High Sensitivity Assay (Invitrogen, Mulgrave, VIC, Australia) on a Qubit ${ }^{\circledR} 2.0$ Fluorometer (Life Technologies, Mulgrave, VIC, Australia) according to the manufacturer's instructions. The limit of detection was $10 \mathrm{pg} / \mu \mathrm{L}$.

\subsection{S rRNA Gene Amplification and Barcoding}

The full-length 16S rRNA gene was amplified using the primer pair 27F and 1492R with a universal UNITAG sequence and amine block attached to the $5^{\prime}$ ends of each primer, as previously described [141,142].

Primary PCR was carried out in $25 \mu \mathrm{L}$ reactions containing $0.3 \mu \mathrm{M}$ each of the forward and reverse primers, 1X AccuStart II ToughMix (Quantabio, Beverly, MA, USA), $0.625 \mu \mathrm{L}$ each of ArcticZymes dsDNase and DTT (ArcticZymes PCR decontamination kit, Tromsø, Norway), $5.5 \mu \mathrm{L}$ nuclease-free water and $5 \mu \mathrm{L}$ of template or nuclease-free water. The activation and inactivation of ArcticZymes dsDNase was performed as described previously [142]. Two negative template controls were included for every 94 samples. The PCR cycling conditions consisted of an initial heating step at $94{ }^{\circ} \mathrm{C}$ for $3 \mathrm{~min}$, followed by $35 \mathrm{cy}-$ cles of $94{ }^{\circ} \mathrm{C}$ for $30 \mathrm{~s}, 52{ }^{\circ} \mathrm{C}$ for $30 \mathrm{~s}$, and $72{ }^{\circ} \mathrm{C}$ for $2 \mathrm{~min}$ and a final extension step of $72{ }^{\circ} \mathrm{C}$ for 5 min. Primary PCR products were visualized on a QIAxcel capillary gel electrophoresis system using a DNA high-resolution cartridge (run parameters OM500) to confirm the presence and size of amplicons. Primary PCR products were purified using NucleoMag NGS magnetic beads (Macherey-Nagel, Düren, Germany), normalized to $1 \mathrm{ng} / \mu \mathrm{L}$ and used as template for the barcoding PCR.

Primary PCR products were barcoded using an asymmetric barcoding strategy. PacBio UNITAG barcoded primers $1 F-8 F$ and $16 \mathrm{R}-30 \mathrm{R}$ were used. PCR reactions were carried out in $20 \mu \mathrm{L}$ volumes containing $0.3 \mu \mathrm{M}$ each of the forward and reverse barcoded primers, $1 X$ AccuStart II ToughMix, and $2 \mu \mathrm{L}$ of template or nuclease-free water (negative template control). PCR cycling conditions were the same as described above, but with 20 cycles.

Barcoded PCR amplicons were pooled in equimolar concentrations based on QIAxcel quantification of the target $\sim 1500 \mathrm{bp}$ band. The pools were gel purified using a QIAquick gel extraction kit (QIAGEN) according to manufacturer's protocol. $500 \mathrm{ng}$ of DNA (pooled amplicons) was used for library preparation and sequencing.

\subsection{PacBio Sequencing}

Purified amplicon pools were sequenced at the Australian Genome Research Facility (AGRF) at The University of Queensland, QLD, Australia. SMRTbell adapters were ligated onto the barcoded PCR products and the libraries were sequenced by Pacific Biosciences single molecule real-time (SMRT) high-fidelity (HiFi) sequencing on two SMRT cells using the PacBio Sequel II System. Raw data were processed using PacBio SMRTLink to generate demultiplexed .fastq files.

\subsection{Sequencing Data Processing}

Full-length 16S rRNA gene sequence data were processed using Mothur v.1.44.3 [143] (as previously described [137]) on the Pople supercomputer at a high-performance computing cluster (Karton, A; The University of Western Australia). Briefly, .fastq files were converted to .fasta files and merged into a single .fasta file. The merged .fasta file was length filtered (1336-1743 bp) and sequences containing homopolymers of $>9$ bases were removed. Sequences were aligned to the SILVA reference alignment v138 and pre-clustered. Chimeric sequences were removed using the chimera.vsearch command. Sequences were then classified using classify.seqs with the SILVA taxonomy database v138 and a confidence threshold of 80 . Based on classification, non-bacterial sequences were filtered and discarded from the dataset. Operational taxonomic units (OTUs) were created using the cluster.split command with a 0.03 similarity cut-off value. Clustered OTUs were assigned taxonomy 
using classify.otu. The Good's coverage for each sample type for raw sequencing data and sequencing depth at 427 reads is provided in Table A1. Subsampling was performed at 427 reads based on an average Good's coverage (collectively for all samples) value of $72.0 \%$, eliminating four samples: Mother (M) 1 day 30 maternal faecal sample (20 reads), M1 day 60 maternal faecal sample (297 reads), M2 day 2-5 maternal faecal sample (16 reads), and M6 day 2-5 HM sample (62 reads). The subsampled data at 427 reads were used for all downstream analyses. Reads from negative extraction controls and negative PCR controls are provided in Table A2.

\subsection{Statistical Analysis}

Data were analysed and graphs generated using the $\mathrm{R}$ environment for statistical computing [144-146]. Maternal and infant demographics are provided as mean \pm standard deviation (minimum-maximum) or $n(\%)$.

Alpha diversity was assessed using the Shannon Index and richness (number of different OTUs). In order to compare across time points and samples, linear mixed models were performed with outcomes of Shannon Index and richness, fixed factors of sample (HM, maternal faecal, infant oral and infant faecal), time (days 2-5, 30, 60, 90 and 120), and their respective interaction, as well as a random effect of participant. Estimated mean differences (MDs), 95\% confidence intervals (CIs) and $p$-values are provided.

Beta diversity was assessed by performing a PERMANOVA on the Bray-Curtis dissimilarity matrix. Fixed factors of sample, time, and their respective interaction were included in the model, as well as a random effect of participant. $p$-values are provided. In order to visualise the dissimilarities, a non-metric multi-dimensional scaling (NMDS) plot is presented which was developed using the Bray-Curtis dissimilarity matrix.

Relative abundances were categorised as Absent (relative abundance of 0 ), Low ( $>0$ to $<0.05)$, Medium (0.05 to 0.3) and High (>0.3). Wilcoxon signed-rank tests for paired data, with the Pratt correction for ties, were used to compare this categorised relative abundance variable between time points. The modelling considered only samples that had paired data for the pairwise comparison of interest, and at least one sample's measures must have differed between the two time points being compared. Additionally, only OTUs that were present at an average relative abundance of $>1 \%$ were considered. $p$-values are provided, as well as a heat-map to visualise the changes in abundance over time.

Linear mixed models were used to assess whether concentrations and intakes of each HMO differed over time and between maternal secretor status. Fixed effects of time and secretor status were included, along with their interactions, as well as a random effect of participant. Contrasts were examined for all pairwise comparisons with Tukey corrections.

To assess the relationship between relative abundances in both infant faecal and infant oral samples with HMO intakes, an ANOVA was performed at each time point with the categorised relative abundance data. Estimated mean differences (MD), standard errors (SE) and $p$-values are provided. Significance for all analyses was considered at the $5 \%$ level.

\section{Conclusions}

In conclusion, we found the maternal gut, $\mathrm{HM}$ and infant oral and gut microbiota are dominated by a small number of bacterial taxa that changed in relative abundance over the first four months of life. Furthermore, the variations in infant oral and faecal bacterial profiles were associated with HMO intakes, the clinical relevance of which, however, is currently unknown, but may have implications for infant microbiota development.

Author Contributions: Conceptualization, A.S.C., L.F.S., C.T.L., M.S.P. and D.T.G.; methodology, A.S.C., A.J.F., A.S.R., Z.G., L.F.S., C.T.L., M.S.P. and D.T.G.; data curation, A.S.C. and Z.G.; formal analysis, A.S.C., B.A.T. and M.L.T.; investigation, A.S.C., L.F.S., C.T.L., M.S.P., Z.G. and D.T.G.; visualization, A.S.C. and M.L.T.; writing-original draft, A.S.C. and M.L.T.; writing-review and editing, L.F.S., C.T.L., M.S.P., L.B., Z.G. and D.T.G.; funding acquisition, D.T.G.; resources, L.B., M.S.P. and D.T.G.; supervision, L.F.S., C.T.L., M.S.P. and D.T.G. All authors have read and agreed to the published version of the manuscript. 
Funding: This research is supported by an unrestricted research grant from Medela AG, administered by The University of Western Australia. A.S.C. is supported by an additional SIRF (Scholarships for International Research Fees) scholarship from The University of Western Australia. M.S.P. is supported by the Women and Infants Research Foundation, and a National Health and Medical Research Council project grant (APP \#1144040).

Institutional Review Board Statement: This study was conducted in accordance with the guidelines of the Declaration of Helsinki and approved by the Human Research Ethics Committee at The University of Western Australia (RA/4/20/4023).

Informed Consent Statement: Informed consent was obtained from all participants involved in the study.

Data Availability Statement: The data presented in this study are available from the corresponding author upon reasonable request.

Acknowledgments: We deeply appreciate the assistance of Amir Karton (The University of Western Australia, Australia) for the supercomputing used to process the large sequencing dataset. We are also grateful to the families who participated in the study and sincerely thank Erika van den Dries for assisting in data and sample collection.

Conflicts of Interest: A.S.C., Z.G., L.F.S., C.T.L. and D.T.G. are supported by an unrestricted research grant from Medela AG, administered by The University of Western Australia. The funding bodies had no role in the design of the study, collection/analysis/interpretation of data, writing of the manuscript, or in the decision to publish the results. Other authors have no conflict of interest to disclose.

\section{Appendix A}

Table A1. Good's coverage for each sample type.

\begin{tabular}{ccc}
\hline Sample Types & $\begin{array}{c}\text { Good's Coverage } \\
\text { (Raw Sequencing Data) }\end{array}$ & $\begin{array}{c}\text { Good's Coverage } \\
\text { (Sequencing Depth 427) }\end{array}$ \\
\hline Maternal faecal & $43.0 \%(0.0-91.6 \%)$ & $38.7 \%(17.7-89.8 \%)$ \\
Human milk & $88.6 \%(71.3-98.1 \%)$ & $82.0 \%(58.2-97.2 \%)$ \\
Infant oral & $86.0 \%(62.6-93.9 \%)$ & $78.3 \%(53.5-90.3 \%)$ \\
Infant faecal & $91.4 \%(79.1-96.5 \%)$ & $85.1 \%(68.1-93.4 \%)$ \\
\hline
\end{tabular}

The data is presented as median (min-max). 
Table A2. Number of reads of bacterial OTUs detected in negative extraction controls $(n=6)$ and negative PCR control $(n=8)$.

\begin{tabular}{|c|c|c|c|c|c|c|c|c|c|c|c|c|c|c|c|}
\hline OTUs & Genus & EC 1 & EC 2 & EC 3 & EC 4 & EC 5 & EC 6 & PCRC 1 & PCRC 2 & PCRC 3 & PCRC 4 & PCRC 5 & PCRC 6 & PCRC 7 & PCRC 8 \\
\hline OTU01 & Streptococcus & 1815 & 4536 & 1 & 1 & 1 & 1 & 3 & 2 & 2 & 2 & 3 & 0 & 1 & 0 \\
\hline OTU02 & Staphylococcus & 3 & 0 & 24 & 0 & 0 & 0 & 0 & 1 & 0 & 0 & 0 & 2 & 2 & 3 \\
\hline OTU03 & Bifidobacterium & 4 & 3 & 4677 & 2032 & 22 & 2 & 0 & 1 & 0 & 0 & 0 & 0 & 1 & 0 \\
\hline OTU04 & Bifidobacterium & 3 & 0 & 1047 & 208 & 1 & 0 & 2 & 0 & 0 & 0 & 0 & 1 & 0 & 2 \\
\hline OTU05 & Streptococcus & 2140 & 470 & 64 & 3 & 2 & 0 & 0 & 0 & 0 & 0 & 2 & 0 & 0 & 1 \\
\hline OTU06 & Escherichia-Shigella & 2 & 0 & 623 & 44 & 217 & 87 & 0 & 0 & 0 & 1 & 1 & 0 & 1 & 1 \\
\hline OTU07 & Gemella & 1925 & 508 & 2 & 1 & 0 & 0 & 1 & 0 & 2 & 1 & 0 & 1 & 0 & 0 \\
\hline OTU09 & Acinetobacter & 165 & 716 & 0 & 1 & 0 & 0 & 0 & 0 & 0 & 0 & 0 & 0 & 1 & 0 \\
\hline OTU10 & Bifidobacterium & 0 & 0 & 22 & 1 & 6 & 1 & 0 & 0 & 1 & 0 & 0 & 0 & 1 & 0 \\
\hline OTU11 & Haemophilus & 232 & 333 & 0 & 0 & 0 & 0 & 0 & 0 & 0 & 0 & 0 & 0 & 0 & 0 \\
\hline OTU13 & Raoultella & 0 & 0 & 947 & 10 & 0 & 0 & 0 & 0 & 0 & 0 & 0 & 0 & 0 & 0 \\
\hline OTU16 & Streptococcus & 7 & 87 & 4 & 1 & 0 & 0 & 0 & 0 & 0 & 0 & 0 & 0 & 0 & 0 \\
\hline OTU18 & Veillonella & 22 & 2 & 0 & 0 & 0 & 0 & 0 & 0 & 0 & 0 & 0 & 0 & 0 & 0 \\
\hline OTU19 & Rothia & 1347 & 147 & 0 & 0 & 0 & 0 & 0 & 0 & 0 & 0 & 0 & 0 & 0 & 0 \\
\hline OTU20 & Bacteroides & 0 & 1 & 53 & 9 & 8 & 1 & 0 & 1 & 0 & 0 & 0 & 0 & 4 & 0 \\
\hline OTU23 & Bacteroides & 1 & 14 & 4 & 30 & 836 & 210 & 0 & 3 & 1 & 0 & 1 & 0 & 102 & 0 \\
\hline OTU24 & Bifidobacterium & 0 & 0 & 104 & 5 & 5 & 0 & 0 & 1 & 0 & 0 & 3 & 0 & 1 & 0 \\
\hline OTU25 & Parabacteroides & 1 & 1 & 1087 & 135 & 8 & 3 & 0 & 0 & 0 & 0 & 0 & 0 & 21 & 0 \\
\hline OTU29 & Enterococcus & 0 & 0 & 37 & 4 & 0 & 0 & 0 & 0 & 0 & 0 & 0 & 0 & 0 & 0 \\
\hline OTU30 & Bacilli_c;RF39_o;RF39_fa;RF39_ge & 0 & 0 & 0 & 0 & 0 & 0 & 0 & 0 & 0 & 0 & 2 & 0 & 8991 & 4 \\
\hline OTU31 & Bifidobacterium & 1 & 0 & 343 & 29 & 0 & 0 & 0 & 0 & 0 & 0 & 0 & 0 & 0 & 0 \\
\hline OTU33 & Phascolarctobacterium & 1 & 1 & 53 & 27 & 27 & 4 & 0 & 0 & 0 & 0 & 0 & 0 & 536 & 0 \\
\hline OTU34 & Corynebacterium & 15 & 0 & 4 & 0 & 0 & 0 & 7289 & 856 & 2 & 0 & 0 & 0 & 0 & 0 \\
\hline OTU35 & Bacilli_c;RF39_o;RF39_fa;RF39_ge & 1 & 0 & 0 & 0 & 0 & 0 & 0 & 0 & 0 & 0 & 3 & 0 & 2408 & 0 \\
\hline OTU41 & Bacteroides & 1 & 3 & 1 & 4 & 92 & 30 & 0 & 0 & 0 & 0 & 0 & 0 & 6 & 0 \\
\hline OTU43 & Faecalibacterium & 1 & 5 & 0 & 36 & 97 & 33 & 0 & 0 & 1 & 0 & 0 & 0 & 23 & 0 \\
\hline OTU45 & Lactobacillus & 124 & 123 & 0 & 1 & 0 & 0 & 0 & 0 & 0 & 0 & 0 & 0 & 0 & 0 \\
\hline OTU48 & Streptococcus & 128 & 53 & 0 & 0 & 0 & 0 & 0 & 0 & 0 & 0 & 0 & 0 & 0 & 0 \\
\hline OTU49 & Streptococcus & 108 & 52 & 1 & 0 & 0 & 0 & 0 & 0 & 0 & 0 & 0 & 0 & 0 & 0 \\
\hline OTU57 & Lachnospiraceae_ge & 0 & 0 & 5 & 0 & 4 & 2 & 0 & 0 & 0 & 0 & 0 & 0 & 51 & 0 \\
\hline OTU61 & Bacteroides & 5 & 1 & 1 & 15 & 181 & 92 & 1 & 2 & 0 & 0 & 0 & 0 & 92 & 0 \\
\hline OTU64 & Bifidobacterium & 2 & 0 & 0 & 0 & 26 & 7 & 2 & 0 & 0 & 0 & 0 & 0 & 2 & 0 \\
\hline
\end{tabular}


Table A2. Cont.

\begin{tabular}{|c|c|c|c|c|c|c|c|c|c|c|c|c|c|c|c|}
\hline OTUs & Genus & EC 1 & EC 2 & EC 3 & EC 4 & EC 5 & EC 6 & PCRC 1 & PCRC 2 & PCRC 3 & PCRC 4 & PCRC 5 & PCRC 6 & PCRC 7 & PCRC 8 \\
\hline OTU68 & Streptococcus & 48 & 21 & 0 & 0 & 0 & 0 & 0 & 0 & 0 & 0 & 0 & 0 & 0 & 0 \\
\hline OTU81 & Streptococcus & 33 & 5 & 0 & 0 & 0 & 0 & 0 & 0 & 0 & 0 & 0 & 0 & 0 & 0 \\
\hline OTU85 & Streptococcus & 58 & 20 & 1 & 0 & 0 & 0 & 0 & 0 & 0 & 0 & 0 & 0 & 0 & 0 \\
\hline OTU90 & Gemella & 20 & 15 & 0 & 0 & 0 & 0 & 0 & 0 & 0 & 0 & 0 & 0 & 0 & 0 \\
\hline OTU97 & Streptococcus & 67 & 44 & 0 & 0 & 0 & 0 & 0 & 0 & 0 & 0 & 0 & 0 & 0 & 0 \\
\hline OTU99 & Bifidobacterium & 0 & 0 & 24 & 0 & 2 & 1 & 0 & 0 & 0 & 0 & 0 & 0 & 0 & 0 \\
\hline OTU109 & Blautia & 0 & 0 & 0 & 0 & 40 & 9 & 1 & 0 & 0 & 1 & 0 & 0 & 0 & 0 \\
\hline OTU112 & Anaerostipes & 0 & 0 & 0 & 5 & 29 & 8 & 2 & 0 & 0 & 0 & 0 & 0 & 2 & 0 \\
\hline OTU114 & Fusicatenibacter & 11 & 1 & 0 & 5 & 41 & 10 & 0 & 0 & 0 & 0 & 0 & 0 & 3 & 0 \\
\hline OTU118 & Erysipelotrichaceae_UCG-003 & 0 & 0 & 0 & 1 & 29 & 16 & 0 & 0 & 0 & 0 & 0 & 0 & 7 & 0 \\
\hline OTU124 & Bifidobacterium & 0 & 0 & 32 & 4 & 2 & 0 & 0 & 0 & 0 & 0 & 0 & 0 & 0 & 0 \\
\hline OTU125 & Streptococcus & 37 & 13 & 0 & 0 & 0 & 0 & 0 & 0 & 0 & 0 & 0 & 0 & 0 & 0 \\
\hline OTU139 & Prevotella & 0 & 43 & 0 & 1 & 0 & 0 & 0 & 0 & 0 & 0 & 0 & 0 & 0 & 0 \\
\hline OTU155 & Streptococcus & 18 & 23 & 0 & 0 & 0 & 0 & 0 & 0 & 0 & 0 & 0 & 0 & 0 & 0 \\
\hline OTU156 & Streptococcus & 27 & 8 & 0 & 0 & 0 & 0 & 0 & 0 & 0 & 0 & 0 & 0 & 0 & 0 \\
\hline OTU167 & Agathobacter & 0 & 0 & 0 & 2 & 23 & 21 & 0 & 0 & 0 & 0 & 1 & 0 & 296 & 0 \\
\hline OTU168 & Enterobacteriaceae_unclassified & 0 & 0 & 52 & 3 & 0 & 0 & 0 & 0 & 0 & 0 & 0 & 0 & 0 & 0 \\
\hline OTU170 & Bacteroides & 0 & 0 & 0 & 2 & 62 & 20 & 0 & 0 & 0 & 0 & 0 & 0 & 24 & 0 \\
\hline OTU203 & Bacteroides & 0 & 1 & 0 & 0 & 38 & 31 & 0 & 0 & 0 & 0 & 0 & 0 & 0 & 0 \\
\hline OTU205 & Bifidobacterium & 1 & 0 & 165 & 15 & 0 & 0 & 0 & 0 & 0 & 0 & 0 & 0 & 0 & 0 \\
\hline OTU206 & Bacteroides & 1 & 3 & 0 & 0 & 26 & 3 & 0 & 0 & 0 & 0 & 0 & 0 & 2 & 0 \\
\hline OTU207 & Subdoligranulum & 0 & 1 & 0 & 2 & 21 & 8 & 0 & 0 & 0 & 0 & 0 & 0 & 1 & 0 \\
\hline OTU213 & Parasutterella & 0 & 3 & 0 & 0 & 112 & 29 & 0 & 0 & 0 & 0 & 0 & 0 & 0 & 0 \\
\hline OTU217 & Gemella & 21 & 0 & 0 & 0 & 0 & 0 & 0 & 0 & 0 & 0 & 0 & 0 & 0 & 0 \\
\hline OTU226 & Prevotella & 0 & 112 & 0 & 0 & 0 & 0 & 0 & 0 & 0 & 0 & 0 & 0 & 0 & 0 \\
\hline OTU254 & Parabacteroides & 0 & 0 & 20 & 0 & 0 & 0 & 0 & 0 & 0 & 0 & 0 & 0 & 0 & 0 \\
\hline OTU262 & Enterobacteriaceae_unclassified & 0 & 0 & 28 & 2 & 0 & 0 & 0 & 0 & 0 & 0 & 0 & 0 & 0 & 0 \\
\hline OTU272 & Bifidobacterium & 0 & 1 & 70 & 16 & 0 & 0 & 0 & 0 & 0 & 0 & 0 & 0 & 0 & 0 \\
\hline OTU276 & Lachnospiraceae_ge & 1 & 0 & 1 & 4 & 21 & 9 & 0 & 0 & 0 & 0 & 0 & 0 & 2 & 0 \\
\hline OTU285 & Enterobacteriaceae_unclassified & 0 & 0 & 28 & 2 & 0 & 0 & 0 & 0 & 0 & 0 & 0 & 0 & 0 & 0 \\
\hline OTU292 & Bifidobacterium & 0 & 0 & 48 & 20 & 0 & 0 & 0 & 0 & 0 & 0 & 0 & 0 & 0 & 0 \\
\hline OTU300 & Bifidobacterium & 0 & 0 & 66 & 17 & 0 & 0 & 0 & 0 & 0 & 0 & 0 & 0 & 0 & 0 \\
\hline OTU303 & Bifidobacterium & 0 & 0 & 33 & 4 & 0 & 0 & 0 & 0 & 0 & 0 & 0 & 0 & 0 & 0 \\
\hline
\end{tabular}


Table A2. Cont.

\begin{tabular}{|c|c|c|c|c|c|c|c|c|c|c|c|c|c|c|c|}
\hline OTUs & Genus & EC 1 & EC 2 & EC 3 & EC 4 & EC 5 & EC 6 & PCRC 1 & PCRC 2 & PCRC 3 & PCRC 4 & PCRC 5 & PCRC 6 & PCRC 7 & PCRC 8 \\
\hline OTU306 & Bifidobacterium & 0 & 0 & 38 & 3 & 0 & 0 & 0 & 0 & 0 & 0 & 0 & 0 & 0 & 0 \\
\hline OTU318 & Enterobacteriaceae_unclassified & 0 & 0 & 30 & 0 & 0 & 0 & 0 & 0 & 0 & 0 & 0 & 0 & 0 & 0 \\
\hline OTU322 & Enterobacteriaceae_unclassified & 0 & 0 & 22 & 0 & 0 & 0 & 0 & 0 & 0 & 0 & 0 & 0 & 0 & 0 \\
\hline OTU357 & Acinetobacter & 2 & 21 & 0 & 0 & 0 & 0 & 0 & 0 & 0 & 0 & 0 & 0 & 0 & 0 \\
\hline OTU367 & Bifidobacterium & 0 & 0 & 41 & 4 & 0 & 0 & 0 & 0 & 0 & 0 & 0 & 0 & 0 & 0 \\
\hline OTU380 & Bifidobacterium & 0 & 0 & 29 & 7 & 0 & 0 & 0 & 0 & 0 & 0 & 0 & 0 & 0 & 0 \\
\hline OTU462 & Campylobacter & 0 & 162 & 0 & 0 & 0 & 0 & 0 & 0 & 0 & 0 & 0 & 0 & 0 & 0 \\
\hline OTU558 & Bacilli_c;RF39_o;RF39_fa;RF39_ge & 0 & 0 & 0 & 0 & 0 & 0 & 0 & 0 & 0 & 0 & 0 & 0 & 126 & 0 \\
\hline OTU624 & Bacilli_c;RF39_o;RF39_fa;RF39_ge & 0 & 0 & 0 & 0 & 0 & 0 & 0 & 0 & 0 & 0 & 0 & 0 & 111 & 0 \\
\hline OTU694 & Faecalibacterium & 0 & 0 & 0 & 0 & 24 & 3 & 0 & 0 & 0 & 0 & 0 & 0 & 0 & 0 \\
\hline OTU726 & Bacilli_c;RF39_o;RF39_fa;RF39_ge & 0 & 0 & 0 & 0 & 0 & 0 & 0 & 0 & 0 & 0 & 0 & 0 & 84 & 0 \\
\hline OTU795 & Bacteroides & 0 & 0 & 0 & 0 & 32 & 11 & 0 & 0 & 0 & 0 & 0 & 0 & 0 & 0 \\
\hline OTU942 & Bacilli_c;RF39_o;RF39_fa;RF39_ge & 0 & 0 & 0 & 0 & 0 & 0 & 0 & 0 & 0 & 0 & 0 & 0 & 64 & 0 \\
\hline OTU1372 & Bacilli_c;RF39_o;RF39_fa;RF39_ge & 0 & 0 & 0 & 0 & 0 & 0 & 0 & 0 & 0 & 0 & 0 & 0 & 37 & 0 \\
\hline OTU1827 & Bacilli_c;RF39_o;RF39_fa;RF39_ge & 0 & 0 & 0 & 0 & 0 & 0 & 0 & 0 & 0 & 0 & 0 & 0 & 28 & 0 \\
\hline OTU2087 & Bacilli_c;RF39_o;RF39_fa;RF39_ge & 0 & 0 & 0 & 0 & 0 & 0 & 0 & 0 & 0 & 0 & 0 & 0 & 26 & 0 \\
\hline Others & Others & 3283 & 2255 & 3614 & 728 & 4727 & 1681 & 80 & 59 & 15 & 5 & 20 & 7 & 2763 & 5 \\
\hline
\end{tabular}

"Others" represents OTUs (operational taxonomic unit) accounting for $\leq 20$ reads in negative controls. EC—extraction control; PCRC-polymerase chain reaction control. 
Table A3. The proportion of mothers and infants at each time point for which an OTU was present.

\begin{tabular}{|c|c|c|c|c|c|c|}
\hline \multirow{2}{*}{ OTUs } & \multirow{2}{*}{ Category } & Day 2-5 & Day 30 & Day 60 & Day 90 & Day 120 \\
\hline & & \multicolumn{5}{|c|}{ Maternal Faecal } \\
\hline \multirow{3}{*}{ OTU01 (Streptococcus mitis) } & Absent & $9(100 \%)$ & $9(100 \%)$ & $5(55.56 \%)$ & $7(70 \%)$ & $8(80 \%)$ \\
\hline & Low & $0(0 \%)$ & $0(0 \%)$ & $3(33.33 \%)$ & $3(30 \%)$ & $2(20 \%)$ \\
\hline & High & $0(0 \%)$ & $0(0 \%)$ & $1(11.11 \%)$ & $0(0 \%)$ & $0(0 \%)$ \\
\hline \multirow{3}{*}{ OTU23 (Phocaeicola vulgatus) } & Absent & $2(22.22 \%)$ & $0(0 \%)$ & $1(11.11 \%)$ & $1(10 \%)$ & $2(20 \%)$ \\
\hline & Low & $5(55.56 \%)$ & $3(33.33 \%)$ & $3(33.33 \%)$ & $5(50 \%)$ & $5(50 \%)$ \\
\hline & Medium & $2(22.22 \%)$ & $6(66.67 \%)$ & $5(55.56 \%)$ & $4(40 \%)$ & $3(30 \%)$ \\
\hline \multirow{3}{*}{ OTU43 (Faecalibacterium prausnitzii) } & Absent & $4(44.44 \%)$ & $0(0 \%)$ & $1(11.11 \%)$ & $3(30 \%)$ & $1(10 \%)$ \\
\hline & Low & $5(55.56 \%)$ & $6(66.67 \%)$ & $5(55.56 \%)$ & $6(60 \%)$ & $8(80 \%)$ \\
\hline & Medium & $0(0 \%)$ & $3(33.33 \%)$ & $3(33.33 \%)$ & $1(10 \%)$ & $1(10 \%)$ \\
\hline \multirow{3}{*}{ OTU64 (Bifidobacterium adolescentis) } & Absent & $5(55.56 \%)$ & $5(55.56 \%)$ & $6(66.67 \%)$ & $4(40 \%)$ & $8(80 \%)$ \\
\hline & Low & $3(33.33 \%)$ & $3(33.33 \%)$ & $3(33.33 \%)$ & $4(40 \%)$ & $1(10 \%)$ \\
\hline & Medium & $1(11.11 \%)$ & $1(11.11 \%)$ & $0(0 \%)$ & $2(20 \%)$ & $1(10 \%)$ \\
\hline \multirow{3}{*}{ OTU82 (Oscillibacter sp.) } & Absent & $6(66.67 \%)$ & $7(77.78 \%)$ & $6(66.67 \%)$ & $10(100 \%)$ & $6(60 \%)$ \\
\hline & Low & $2(22.22 \%)$ & $2(22.22 \%)$ & $3(33.33 \%)$ & $0(0 \%)$ & $3(30 \%)$ \\
\hline & Medium & $1(11.11 \%)$ & $0(0 \%)$ & $0(0 \%)$ & $0(0 \%)$ & $1(10 \%)$ \\
\hline \multirow{4}{*}{ OTU107 (Romboutsia timonensis) } & Absent & $7(77.78 \%)$ & $5(55.56 \%)$ & $5(55.56 \%)$ & $2(20 \%)$ & $5(50 \%)$ \\
\hline & Low & $1(11.11 \%)$ & $4(44.44 \%)$ & $4(44.44 \%)$ & $7(70 \%)$ & $5(50 \%)$ \\
\hline & Medium & $1(11.11 \%)$ & $0(0 \%)$ & $0(0 \%)$ & $1(10 \%)$ & $0(0 \%)$ \\
\hline & & \multicolumn{5}{|c|}{ Human Milk } \\
\hline \multirow{4}{*}{ OTU02 (Staphylococcus epidermidis) } & Absent & $1(11.11 \%)$ & $0(0 \%)$ & $2(20 \%)$ & $1(10 \%)$ & $2(20 \%)$ \\
\hline & Low & $3(33.33 \%)$ & $1(10 \%)$ & $5(50 \%)$ & $4(40 \%)$ & $2(20 \%)$ \\
\hline & Medium & $2(22.22 \%)$ & $3(30 \%)$ & $0(0 \%)$ & $3(30 \%)$ & $5(50 \%)$ \\
\hline & High & $3(33.33 \%)$ & $6(60 \%)$ & $3(30 \%)$ & $2(20 \%)$ & $1(10 \%)$ \\
\hline \multirow{4}{*}{ OTU05 (Streptococcus salivarius) } & Absent & $1(11.11 \%)$ & $3(30 \%)$ & $1(10 \%)$ & $1(10 \%)$ & $1(10 \%)$ \\
\hline & Low & $5(55.56 \%)$ & $5(50 \%)$ & $3(30 \%)$ & $4(40 \%)$ & $6(60 \%)$ \\
\hline & Medium & $2(22.22 \%)$ & $2(20 \%)$ & $3(30 \%)$ & $3(30 \%)$ & $2(20 \%)$ \\
\hline & High & $1(11.11 \%)$ & $0(0 \%)$ & $3(30 \%)$ & $2(20 \%)$ & $1(10 \%)$ \\
\hline \multirow{4}{*}{ OTU09 (Acinetobacter johnsonii) } & Absent & $9(100 \%)$ & $0(0 \%)$ & $4(40 \%)$ & $0(0 \%)$ & $10(100 \%)$ \\
\hline & Low & $0(0 \%)$ & $0(0 \%)$ & $4(40 \%)$ & $0(0 \%)$ & $0(0 \%)$ \\
\hline & Medium & $0(0 \%)$ & $0(0 \%)$ & $1(10 \%)$ & $0(0 \%)$ & $0(0 \%)$ \\
\hline & High & $0(0 \%)$ & $0(0 \%)$ & $1(10 \%)$ & $0(0 \%)$ & $0(0 \%)$ \\
\hline \multirow{3}{*}{ OTU14 (Veillonella nakazawae) } & Absent & $9(100 \%)$ & $6(60 \%)$ & $7(70 \%)$ & $8(80 \%)$ & $5(50 \%)$ \\
\hline & Low & $0(0 \%)$ & $4(40 \%)$ & $3(30 \%)$ & $2(20 \%)$ & $4(40 \%)$ \\
\hline & Medium & $0(0 \%)$ & $0(0 \%)$ & $0(0 \%)$ & $0(0 \%)$ & $1(10 \%)$ \\
\hline \multirow{3}{*}{ OTU16 (Streptococcus lactarius) } & Absent & $7(77.78 \%)$ & $8(80 \%)$ & $6(60 \%)$ & $7(70 \%)$ & $5(50 \%)$ \\
\hline & Low & $2(22.22 \%)$ & $1(10 \%)$ & $3(30 \%)$ & $1(10 \%)$ & $2(20 \%)$ \\
\hline & Medium & $0(0 \%)$ & $1(10 \%)$ & $1(10 \%)$ & $2(20 \%)$ & $3(30 \%)$ \\
\hline \multirow{3}{*}{ OTU26 (Dolosigranulum pigrum) } & Absent & $7(77.78 \%)$ & $9(90 \%)$ & $10(100 \%)$ & $7(70 \%)$ & $6(60 \%)$ \\
\hline & Low & $2(22.22 \%)$ & $1(10 \%)$ & $0(0 \%)$ & $1(10 \%)$ & $4(40 \%)$ \\
\hline & Medium & $0(0 \%)$ & $0(0 \%)$ & $0(0 \%)$ & $2(20 \%)$ & $0(0 \%)$ \\
\hline \multirow{3}{*}{ OTU28 (Staphyloccocus hominis) } & Absent & $5(55.56 \%)$ & $9(90 \%)$ & $6(60 \%)$ & $8(80 \%)$ & $5(50 \%)$ \\
\hline & Low & $3(33.33 \%)$ & $1(10 \%)$ & $4(40 \%)$ & $2(20 \%)$ & $5(50 \%)$ \\
\hline & Medium & $1(11.11 \%)$ & $0(0 \%)$ & $0(0 \%)$ & $0(0 \%)$ & $0(0 \%)$ \\
\hline
\end{tabular}


Table A3. Cont.

\begin{tabular}{|c|c|c|c|c|c|c|}
\hline \multirow{2}{*}{ OTUs } & \multirow{2}{*}{ Category } & Day 2-5 & Day 30 & Day 60 & Day 90 & Day 120 \\
\hline & & \multicolumn{5}{|c|}{ Infant Oral } \\
\hline \multirow{4}{*}{ OTU01 (Streptococcus mitis) } & Absent & $1(10 \%)$ & $0(0 \%)$ & $0(0 \%)$ & $0(0 \%)$ & $0(0 \%)$ \\
\hline & Low & $3(30 \%)$ & $4(40 \%)$ & $4(40 \%)$ & $1(10 \%)$ & $1(10 \%)$ \\
\hline & Medium & $6(60 \%)$ & $4(40 \%)$ & $2(20 \%)$ & $2(20 \%)$ & $3(30 \%)$ \\
\hline & High & $0(0 \%)$ & $2(20 \%)$ & $4(40 \%)$ & $7(70 \%)$ & $6(60 \%)$ \\
\hline \multirow{4}{*}{ OTU02 (Staphylococcus epidermidis) } & Absent & $4(40 \%)$ & $5(50 \%)$ & $8(80 \%)$ & $9(90 \%)$ & $10(100 \%)$ \\
\hline & Low & $2(20 \%)$ & $5(50 \%)$ & $2(20 \%)$ & $1(10 \%)$ & $0(0 \%)$ \\
\hline & Medium & $3(30 \%)$ & $0(0 \%)$ & $0(0 \%)$ & $0(0 \%)$ & $0(0 \%)$ \\
\hline & High & $1(10 \%)$ & $0(0 \%)$ & $0(0 \%)$ & $0(0 \%)$ & $0(0 \%)$ \\
\hline \multirow{4}{*}{ OTU05 (Streptococcus salivarius) } & Absent & $5(50 \%)$ & $2(20 \%)$ & $4(40 \%)$ & $4(40 \%)$ & $8(80 \%)$ \\
\hline & Low & $3(30 \%)$ & $7(70 \%)$ & $4(40 \%)$ & $5(50 \%)$ & $1(10 \%)$ \\
\hline & Medium & $1(10 \%)$ & $0(0 \%)$ & $1(10 \%)$ & $1(10 \%)$ & $1(10 \%)$ \\
\hline & High & $1(10 \%)$ & $1(10 \%)$ & $1(10 \%)$ & $0(0 \%)$ & $0(0 \%)$ \\
\hline \multirow{4}{*}{ OTU11 (Haemophilus haemolyticus) } & Absent & $2(20 \%)$ & $6(60 \%)$ & $6(60 \%)$ & $7(70 \%)$ & $2(20 \%)$ \\
\hline & Low & $7(70 \%)$ & $2(20 \%)$ & $1(10 \%)$ & $1(10 \%)$ & $4(40 \%)$ \\
\hline & Medium & $0(0 \%)$ & $1(10 \%)$ & $3(30 \%)$ & $2(20 \%)$ & $3(30 \%)$ \\
\hline & High & $1(10 \%)$ & $1(10 \%)$ & $0(0 \%)$ & $0(0 \%)$ & $1(10 \%)$ \\
\hline \multirow{4}{*}{ OTU14 (Veillonella nakazawae) } & Absent & $9(90 \%)$ & $5(50 \%)$ & $7(70 \%)$ & $6(60 \%)$ & $2(20 \%)$ \\
\hline & Low & $1(10 \%)$ & $2(20 \%)$ & $2(20 \%)$ & $3(30 \%)$ & $8(80 \%)$ \\
\hline & Medium & $0(0 \%)$ & $2(20 \%)$ & $1(10 \%)$ & $1(10 \%)$ & $0(0 \%)$ \\
\hline & High & $0(0 \%)$ & $1(10 \%)$ & $0(0 \%)$ & $0(0 \%)$ & $0(0 \%)$ \\
\hline \multirow{3}{*}{ OTU16 (Streptococcus lactarius) } & Absent & $8(80 \%)$ & $9(90 \%)$ & $6(60 \%)$ & $6(60 \%)$ & $3(30 \%)$ \\
\hline & Low & $2(20 \%)$ & $1(10 \%)$ & $4(40 \%)$ & $3(30 \%)$ & $6(60 \%)$ \\
\hline & Medium & $0(0 \%)$ & $0(0 \%)$ & $0(0 \%)$ & $1(10 \%)$ & $1(10 \%)$ \\
\hline \multirow{3}{*}{ OTU28 (Staphyloccocus hominis) } & Absent & $5(50 \%)$ & $8(80 \%)$ & $9(90 \%)$ & $10(100 \%)$ & $10(100 \%)$ \\
\hline & Low & $4(40 \%)$ & $2(20 \%)$ & $1(10 \%)$ & $0(0 \%)$ & $0(0 \%)$ \\
\hline & Medium & $1(10 \%)$ & $0(0 \%)$ & $0(0 \%)$ & $0(0 \%)$ & $0(0 \%)$ \\
\hline & & \multicolumn{5}{|c|}{ Infant Faecal } \\
\hline \multirow{4}{*}{ OTU02 (Staphylococcus epidermidis) } & Absent & $2(20 \%)$ & $3(30 \%)$ & $3(30 \%)$ & $6(60 \%)$ & $7(70 \%)$ \\
\hline & Low & $5(50 \%)$ & $6(60 \%)$ & $6(60 \%)$ & $4(40 \%)$ & $3(30 \%)$ \\
\hline & Medium & $3(30 \%)$ & $1(10 \%)$ & $1(10 \%)$ & $0(0 \%)$ & $0(0 \%)$ \\
\hline & Absent & $3(30 \%)$ & $1(10 \%)$ & $5(50 \%)$ & $5(50 \%)$ & $7(70 \%)$ \\
\hline \multirow{3}{*}{ OTU05 (Streptococcus salivarius) } & Low & $4(40 \%)$ & $7(70 \%)$ & $4(40 \%)$ & $5(50 \%)$ & $3(30 \%)$ \\
\hline & Medium & $3(30 \%)$ & $1(10 \%)$ & $1(10 \%)$ & $0(0 \%)$ & $0(0 \%)$ \\
\hline & High & $0(0 \%)$ & $1(10 \%)$ & $0(0 \%)$ & $0(0 \%)$ & $0(0 \%)$ \\
\hline \multirow{3}{*}{ OTU14 (Veillonella nakazawae) } & Absent & $9(90 \%)$ & $10(100 \%)$ & $5(50 \%)$ & $8(80 \%)$ & $6(60 \%)$ \\
\hline & Low & $0(0 \%)$ & $0(0 \%)$ & $5(50 \%)$ & $1(10 \%)$ & $2(20 \%)$ \\
\hline & Medium & $1(10 \%)$ & $0(0 \%)$ & $0(0 \%)$ & $1(10 \%)$ & $2(20 \%)$ \\
\hline \multirow{4}{*}{ OTU20 (Bacteroides fragilis) } & Absent & $10(100 \%)$ & $8(80 \%)$ & $4(40 \%)$ & $10(100 \%)$ & $8(80 \%)$ \\
\hline & Low & $0(0 \%)$ & $2(20 \%)$ & $5(50 \%)$ & $0(0 \%)$ & $1(10 \%)$ \\
\hline & Medium & $0(0 \%)$ & $0(0 \%)$ & $0(0 \%)$ & $0(0 \%)$ & $1(10 \%)$ \\
\hline & High & $0(0 \%)$ & $0(0 \%)$ & $1(10 \%)$ & $0(0 \%)$ & $0(0 \%)$ \\
\hline \multirow{3}{*}{ OTU24 (Bifidobacterium longum) } & Absent & $3(30 \%)$ & $7(70 \%)$ & $4(40 \%)$ & $6(60 \%)$ & $7(70 \%)$ \\
\hline & Low & $3(30 \%)$ & $1(10 \%)$ & $4(40 \%)$ & $4(40 \%)$ & $2(20 \%)$ \\
\hline & Medium & $4(40 \%)$ & $2(20 \%)$ & $2(20 \%)$ & $0(0 \%)$ & $1(10 \%)$ \\
\hline
\end{tabular}

Results are presented as the number of participants having OTUs (operational taxonomic unit) (percentage of participants). 
Table A4. Associations between individual HMO intake and infant oral bacterial composition.

\begin{tabular}{|c|c|c|c|c|}
\hline \multirow{2}{*}{ Bacterial OTUs } & \multirow{2}{*}{$\begin{array}{c}\text { Comparison } \\
\text { (Parameter-Intercept) }\end{array}$} & Estimated Difference & SE & $p$-Value \\
\hline & & \multicolumn{3}{|c|}{ Day 30} \\
\hline & & \multicolumn{3}{|c|}{ LNnT } \\
\hline OTU11 (Haemophilus haemolyticus) & Low-Absent & 83.375 & 30.368 & 0.034 \\
\hline \multirow[t]{2}{*}{ OTU11 (Haemophilus haemolyticus) } & High-Absent & 240.073 & 40.174 & 0.001 \\
\hline & & \multicolumn{3}{|c|}{ LNFP I } \\
\hline OTU18 (Veillonella sp. oral clone ASCB03) & High-Absent & 1541.626 & 351.386 & 0.003 \\
\hline \multirow[t]{2}{*}{ OTU32 (Haemophilus parainfluenzae) } & Low-Absent & 1502.522 & 332.410 & 0.003 \\
\hline & & \multicolumn{3}{|c|}{ LNFP III } \\
\hline OTU18 (Veillonella sp. oral clone ASCB03) & High-Absent & 44.042 & 3.996 & $<0.001$ \\
\hline \multirow[t]{2}{*}{ OTU32 (Haemophilus parainfluenzae) } & Low-Absent & 44.201 & 3.754 & $<0.001$ \\
\hline & & \multicolumn{3}{|c|}{ LSTc } \\
\hline OTU18 (Veillonella sp. oral clone ASCB03) & High-Absent & 233.322 & 40.045 & $<0.001$ \\
\hline \multirow[t]{2}{*}{ OTU32 (Haemophilus parainfluenzae) } & Low-Absent & 245.268 & 31.792 & $<0.001$ \\
\hline & & \multicolumn{3}{|c|}{ LNH } \\
\hline \multirow[t]{2}{*}{ OTU11 (Haemophilus haemolyticus) } & High-Absent & 64.315 & 17.900 & 0.012 \\
\hline & & \multicolumn{3}{|c|}{ DSLNT } \\
\hline OTU11 (Haemophilus haemolyticus) & Low-Absent & 113.917 & 28.901 & 0.008 \\
\hline OTU18 (Veillonella sp. oral clone ASCB03) & Low-Absent & -51.026 & 20.311 & 0.040 \\
\hline OTU18 (Veillonella sp. oral clone ASCB03) & High-Absent & 110.395 & 33.168 & 0.013 \\
\hline \multirow[t]{2}{*}{ OTU32 (Haemophilus parainfluenzae) } & Low-Absent & 124.808 & 34.216 & 0.008 \\
\hline & & \multicolumn{3}{|c|}{ FLNH } \\
\hline \multirow[t]{3}{*}{ OTU05 (Streptococcus salivarius) } & High-Absent & 323.804 & 94.591 & 0.011 \\
\hline & & \multicolumn{3}{|c|}{ Day 60} \\
\hline & & \multicolumn{3}{|c|}{$3 F L$} \\
\hline \multirow[t]{2}{*}{ OTU02 (Staphylococcus epidermidis) } & Low-Absent & -550.146 & 203.242 & 0.027 \\
\hline & & \multicolumn{3}{|c|}{ DFLac } \\
\hline \multirow[t]{2}{*}{ OTU32 (Haemophilus parainfluenzae) } & Medium-Absent & 224.329 & 70.758 & 0.016 \\
\hline & & \multicolumn{3}{|c|}{ LNnT } \\
\hline \multirow[t]{2}{*}{ OTU03 (Bifidobacterium longum subsp. infantis) } & Low-Absent & 100.502 & 31.407 & 0.015 \\
\hline & & \multicolumn{3}{|c|}{ LNFP I } \\
\hline OTU18 (Veillonella sp. oral clone ASCB03) & Medium-Absent & 788.647 & 204.195 & 0.006 \\
\hline & & $\mathrm{LNl}$ & & \\
\hline OTU02 (Staphylococcus epidermidis) & Low-Absent & -381.626 & 155.937 & 0.040 \\
\hline & & LNF & & \\
\hline OTU18 (Veillonella sp. oral clone ASCB03) & Medium-Absent & 11.980 & 3.171 & 0.007 \\
\hline & & LS & & \\
\hline OTU14 (Veillonella nakazawae) & Low-Absent & 42.776 & 15.187 & 0.026 \\
\hline & & $\mathrm{LS}$ & & \\
\hline OTU01 (Streptococcus mitis) & Medium-Low & 69.136 & 17.701 & 0.006 \\
\hline & & $\mathrm{DFI}$ & & \\
\hline OTU14 (Veillonella nakazawae) & Low-Absent & 553.393 & 176.628 & 0.017 \\
\hline & & DSI & & \\
\hline OTU14 (Veillonella nakazawae) & Low-Absent & 70.404 & 20.949 & 0.012 \\
\hline
\end{tabular}


Table A4. Cont.

\begin{tabular}{|c|c|c|c|c|}
\hline \multirow{2}{*}{ Bacterial OTUs } & \multirow{2}{*}{$\begin{array}{c}\text { Comparison } \\
\text { (Parameter-Intercept) }\end{array}$} & Estimated Difference & SE & $p$-Value \\
\hline & & \multicolumn{3}{|c|}{ Day 90} \\
\hline & & \multicolumn{3}{|c|}{ DFLac } \\
\hline OTU01 (Streptococcus mitis) & Medium-Low & 326.879 & 98.311 & 0.013 \\
\hline \multirow[t]{2}{*}{ OTU32 (Haemophilus parainfluenzae) } & Medium-Absent & 321.837 & 79.181 & 0.004 \\
\hline & & \multicolumn{3}{|c|}{ 3'SL } \\
\hline OTU01 (Streptococcus mitis) & Medium-Low & 115.877 & 48.265 & 0.047 \\
\hline \multirow[t]{2}{*}{ OTU32 (Haemophilus parainfluenzae) } & Medium-Absent & 167.484 & 20.209 & $<0.001$ \\
\hline & & \multicolumn{3}{|c|}{$6^{\prime} \mathrm{SL}$} \\
\hline OTU19 (Rothia mucilaginosa) & Low-Absent & -60.645 & 18.879 & 0.015 \\
\hline \multirow{2}{*}{ OTU19 (Rothia mucilaginosa) } & Medium-Absent & -85.766 & 30.830 & 0.027 \\
\hline & & \multicolumn{3}{|c|}{ LNT } \\
\hline \multirow[t]{2}{*}{ OTU05 (Streptococcus salivarius) } & Low-Absent & -275.077 & 47.665 & $<0.001$ \\
\hline & & \multicolumn{3}{|c|}{ LNFP I } \\
\hline \multirow[t]{2}{*}{ OTU18 (Veillonella sp. oral clone ASCB03) } & Medium-Absent & 242.589 & 62.818 & 0.008 \\
\hline & & \multicolumn{3}{|c|}{ LNFP II } \\
\hline \multirow[t]{2}{*}{ OTU22 (Bergeyella sp.) } & Low-Absent & -251.985 & 90.486 & 0.024 \\
\hline & & \multicolumn{3}{|c|}{$\mathrm{LSTb}$} \\
\hline OTU07 (Gemella haemolysans) & Low-Absent & -39.158 & 10.458 & 0.007 \\
\hline \multirow[t]{2}{*}{ OTU07 (Gemella haemolysans) } & Medium-Absent & -28.050 & 9.783 & 0.024 \\
\hline & & \multicolumn{3}{|c|}{ LSTc } \\
\hline \multirow[t]{2}{*}{ OTU32 (Haemophilus parainfluenzae) } & Medium-Absent & 68.280 & 18.015 & 0.005 \\
\hline & & \multicolumn{3}{|c|}{ DSLNT } \\
\hline OTU07 (Gemella haemolysans) & Low-Absent & -56.769 & 14.608 & 0.006 \\
\hline OTU07 (Gemella haemolysans) & Medium-Absent & -44.494 & 13.664 & 0.014 \\
\hline \multirow[t]{2}{*}{ OTU18 (Veillonella sp. oral clone ASCB03) } & Medium-Absent & 45.434 & 9.703 & 0.003 \\
\hline & & \multicolumn{3}{|c|}{ DSLNH } \\
\hline \multirow[t]{3}{*}{ OTU19 (Rothia mucilaginosa) } & Low-Absent & -46.494 & 11.472 & 0.005 \\
\hline & & \multicolumn{3}{|c|}{ Day 120} \\
\hline & & \multicolumn{3}{|c|}{$2^{\prime} \mathrm{FL}$} \\
\hline OTU03 (Bifidobacterium longum subsp. infantis) & Low-Absent & 1044.095 & 324.676 & 0.012 \\
\hline OTU18 (Veillonella sp. oral clone ASCB03) & Medium-Absent & 1143.659 & 348.875 & 0.014 \\
\hline & & & & \\
\hline OTU03 (Bifidobacterium longum subsp. infantis) & Low-Absent & -597.110 & 241.857 & 0.039 \\
\hline OTU18 (Veillonella sp. oral clone ASCB03) & Medium-Absent & -736.135 & 221.592 & 0.013 \\
\hline & & $3^{\prime}$ & & \\
\hline OTU32 (Haemophilus parainfluenzae) & Low-Absent & 112.458 & 11.639 & $<0.001$ \\
\hline & & $6^{\prime}$ & & \\
\hline OTU01 (Streptococcus mitis) & High-Low & -58.021 & 20.220 & 0.024 \\
\hline OTU03 (Bifidobacterium longum subsp. infantis) & Low-Absent & 63.180 & 18.410 & 0.009 \\
\hline OTU18 (Veillonella sp. oral clone ASCB03) & Medium-Absent & 67.677 & 20.171 & 0.012 \\
\hline & & LN & & \\
\hline OTU03 (Bifidobacterium longum subsp. infantis) & Low-Absent & 291.768 & 88.867 & 0.011 \\
\hline OTU18 (Veillonella sp. oral clone ASCB03) & Medium-Absent & 323.706 & 93.534 & 0.011 \\
\hline
\end{tabular}


Table A4. Cont.

\begin{tabular}{|c|c|c|c|c|}
\hline \multirow{2}{*}{ Bacterial OTUs } & \multirow{2}{*}{$\begin{array}{c}\text { Comparison } \\
\text { (Parameter-Intercept) }\end{array}$} & Estimated Difference & SE & $p$-Value \\
\hline & & \multicolumn{3}{|c|}{ Day 120} \\
\hline & & \multicolumn{3}{|c|}{$\mathrm{LNH}$} \\
\hline \multirow{2}{*}{$\begin{array}{l}\text { OTU03 (Bifidobacterium longum subsp. infantis) } \\
\text { OTU18 (Veillonella sp. oral clone ASCB03) }\end{array}$} & Low-Absent & 31.952 & 9.363 & 0.009 \\
\hline & Medium-Absent & 32.123 & 10.591 & 0.019 \\
\hline & & \multicolumn{3}{|c|}{ DSLNT } \\
\hline \multirow[t]{2}{*}{ OTU01 (Streptococcus mitis) } & High-Low & -58.388 & 21.383 & 0.029 \\
\hline & & \multicolumn{3}{|c|}{ FDSLNH } \\
\hline \multirow[t]{2}{*}{ OTU05 (Streptococcus salivarius), } & Low-Absent & 307.528 & 96.080 & 0.015 \\
\hline & & \multicolumn{3}{|c|}{ DSLNH } \\
\hline OTU01 (Streptococcus mitis) & High-Low & -22.305 & 8.279 & 0.031 \\
\hline OTU03 (Bifidobacterium longum subsp. infantis) & Low-Absent & 32.307 & 11.696 & 0.025 \\
\hline & \multicolumn{4}{|c|}{ 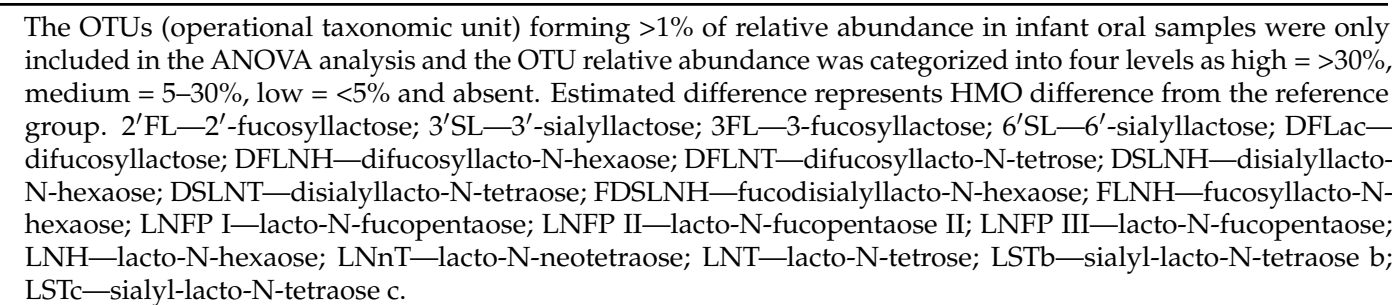 } \\
\hline
\end{tabular}

Table A5. Associations between individual HMO intake and infant faecal bacterial composition.

\begin{tabular}{|c|c|c|c|c|}
\hline \multirow{2}{*}{ Bacterial OTUs } & \multirow{2}{*}{$\begin{array}{c}\text { Comparison } \\
\text { (Parameter-Intercept) }\end{array}$} & Estimated Difference & SE & $p$-Value \\
\hline & & \multicolumn{3}{|c|}{ Day 30} \\
\hline & & \multicolumn{3}{|c|}{$2^{\prime} \mathrm{FL}$} \\
\hline \multirow[t]{2}{*}{ OTU04 (Bifidobacterium breve) } & Low-Absent & -1666.940 & 425.648 & 0.008 \\
\hline & & \multicolumn{3}{|c|}{ 3FL } \\
\hline OTU13 (Raoultella ornithinolytica) & High-Absent & 735.702 & 258.385 & 0.025 \\
\hline \multirow[t]{2}{*}{ OTU24 (Bifidobacterium longum) } & Low-Absent & 723.455 & 265.973 & 0.030 \\
\hline & & \multicolumn{3}{|c|}{ DFLac } \\
\hline \multirow[t]{2}{*}{ OTU04 (Bifidobacterium breve) } & Low-Absent & -138.030 & 28.935 & 0.003 \\
\hline & & \multicolumn{3}{|c|}{$3^{\prime} \mathrm{SL}$} \\
\hline OTU03 (Bifidobacterium longum subsp. infantis) & Medium-Absent & -41.128 & 14.211 & 0.028 \\
\hline \multirow[t]{2}{*}{ OTU20 (Bacteroides fragilis) } & Low-Absent & 48.548 & 19.523 & 0.038 \\
\hline & & \multicolumn{3}{|c|}{ LNnT } \\
\hline OTU03 (Bifidobacterium longum subsp. infantis) & Low-Absent & 205.125 & 55.873 & 0.010 \\
\hline OTU17 (Klebsiella pneumoniae) & Low-Absent & 232.141 & 40.266 & $<0.001$ \\
\hline \multirow[t]{2}{*}{ OTU20 (Bacteroides fragilis) } & Low-Absent & 126.620 & 53.970 & 0.047 \\
\hline & & \multicolumn{3}{|c|}{ LNFP I } \\
\hline OTU05 (Streptococcus salivarius) & Medium-Absent & 1813.872 & 453.887 & 0.007 \\
\hline OTU10 (Bifidobacterium pseudocatenulatum) & High-Absent & 1475.480 & 350.940 & 0.006 \\
\hline OTU17 (Klebsiella pneumoniae) & High-Absent & 1492.947 & 323.246 & 0.002 \\
\hline \multirow[t]{2}{*}{ OTU25 (Parabacteroides distasonis) } & Low-Absent & 1524.848 & 316.978 & 0.001 \\
\hline & & \multicolumn{3}{|c|}{ LNFP II } \\
\hline OTU17 (Klebsiella pneumoniae) & Low-Absent & 484.536 & 170.968 & 0.025 \\
\hline
\end{tabular}


Table A5. Cont.

\begin{tabular}{|c|c|c|c|c|}
\hline \multirow{2}{*}{ Bacterial OTUs } & \multirow{2}{*}{$\begin{array}{c}\text { Comparison } \\
\text { (Parameter-Intercept) }\end{array}$} & Estimated Difference & SE & $p$-Value \\
\hline & & \multicolumn{3}{|c|}{ Day 30} \\
\hline & & \multicolumn{3}{|c|}{ LNFP III } \\
\hline OTU05 (Streptococcus salivarius) & Low-Absent & 5.726 & 2.302 & 0.047 \\
\hline OTU05 (Streptococcus salivarius) & Medium-Absent & 50.291 & 3.045 & $<0.001$ \\
\hline OTU05 (Streptococcus salivarius) & High—Absent & 11.672 & 3.045 & 0.009 \\
\hline OTU10 (Bifidobacterium pseudocatenulatum) & High-Absent & 44.116 & 4.077 & $<0.001$ \\
\hline OTU17 (Klebsiella pneumoniae) & High-Absent & 44.575 & 3.925 & $<0.001$ \\
\hline \multirow[t]{2}{*}{ OTU25 (Parabacteroides distasonis) } & Low-Absent & 44.540 & 3.651 & $<0.001$ \\
\hline & & \multicolumn{3}{|c|}{$\mathrm{LSTb}$} \\
\hline \multirow[t]{2}{*}{ OTU24 (Bifidobacterium longum) } & Low-Absent & 49.510 & 17.349 & 0.025 \\
\hline & & \multicolumn{3}{|c|}{ LSTc } \\
\hline OTU05 (Streptococcus salivarius) & Medium-Absent & 281.536 & 46.123 & $<0.001$ \\
\hline OTU10 (Bifidobacterium pseudocatenulatum) & High-Absent & 247.574 & 33.782 & $<0.001$ \\
\hline OTU17 (Klebsiella pneumoniae) & Low-Absent & 70.097 & 19.648 & 0.009 \\
\hline OTU17 (Klebsiella pneumoniae) & High-Absent & 241.556 & 37.923 & $<0.001$ \\
\hline \multirow[t]{2}{*}{ OTU25 (Parabacteroides distasonis) } & Low-Absent & 238.437 & 36.608 & $<0.001$ \\
\hline & & \multicolumn{3}{|c|}{ DFLNT } \\
\hline \multirow[t]{2}{*}{ OTU04 (Bifidobacterium breve) } & Low-Absent & -597.364 & 136.154 & 0.005 \\
\hline & & \multicolumn{3}{|c|}{ DSLNT } \\
\hline OTU10 (Bifidobacterium pseudocatenulatum) & High-Absent & 124.563 & 37.241 & 0.016 \\
\hline \multirow[t]{2}{*}{ OTU25 (Parabacteroides distasonis) } & Low-Absent & 133.074 & 41.169 & 0.012 \\
\hline & & \multicolumn{3}{|c|}{ FLNH } \\
\hline OTU13 (Raoultella ornithinolytica) & High-Absent & 342.258 & 81.920 & 0.004 \\
\hline \multirow[t]{2}{*}{ OTU24 (Bifidobacterium longum) } & Low-Absent & 351.494 & 83.120 & 0.004 \\
\hline & & \multicolumn{3}{|c|}{ DFLNH } \\
\hline \multirow[t]{2}{*}{ OTU20 (Bacteroides fragilis) } & Low-Absent & 88.416 & 37.035 & 0.044 \\
\hline & & \multicolumn{3}{|c|}{ DSLNH } \\
\hline \multirow[t]{3}{*}{ OTU20 (Bacteroides fragilis) } & Low-Absent & 35.677 & 15.099 & 0.046 \\
\hline & & \multicolumn{3}{|c|}{ Day 60} \\
\hline & & \multicolumn{3}{|c|}{$3 F L$} \\
\hline OTU05 (Streptococcus salivarius) & Low-Absent & -457.444 & 140.352 & 0.014 \\
\hline \multirow[t]{2}{*}{ OTU05 (Streptococcus salivarius) } & Medium-Absent & -721.382 & 229.193 & 0.016 \\
\hline & & \multicolumn{3}{|c|}{ LNT } \\
\hline OTU06 (Escherichia coli) & Medium-Absent & 498.920 & 145.761 & 0.014 \\
\hline \multirow[t]{2}{*}{ OTU29 (Enterococcus faecalis) } & Low-Absent & 636.978 & 249.903 & 0.034 \\
\hline & & $\mathrm{LN}$ & & \\
\hline OTU17 (Klebsiella pneumoniae) & Low-Absent & 76.183 & 23.105 & 0.013 \\
\hline OTU17 (Klebsiella pneumoniae) & Medium-Absent & 70.373 & 23.105 & 0.019 \\
\hline & & LN & & \\
\hline OTU25 (Parabacteroides distasonis) & Low-Absent & 700.864 & 210.358 & 0.013 \\
\hline & & $\mathrm{LN}$ & & \\
\hline OTU29 (Enterococcus faecalis) & Low-Absent & 520.197 & 204.372 & 0.034 \\
\hline & & LNF & III & \\
\hline OTU25 (Parabacteroides distasonis) & Low-Absent & 11.677 & 2.660 & 0.003 \\
\hline
\end{tabular}


Table A5. Cont.

\begin{tabular}{|c|c|c|c|c|}
\hline \multirow{2}{*}{ Bacterial OTUs } & \multirow{2}{*}{$\begin{array}{c}\text { Comparison } \\
\text { (Parameter-Intercept) }\end{array}$} & Estimated Difference & SE & $p$-Value \\
\hline & & \multicolumn{3}{|c|}{ Day 60} \\
\hline & & \multicolumn{3}{|c|}{$\mathrm{LSTb}$} \\
\hline OTU06 (Escherichia coli) & Medium-Absent & 39.951 & 11.096 & 0.011 \\
\hline \multirow[t]{2}{*}{ OTU25 (Parabacteroides distasonis) } & Low-Absent & 55.080 & 20.155 & 0.029 \\
\hline & & \multicolumn{3}{|c|}{ DFLNT } \\
\hline \multirow[t]{2}{*}{ OTU25 (Parabacteroides distasonis) } & Low-Absent & 666.649 & 211.012 & 0.016 \\
\hline & & \multicolumn{3}{|c|}{$\mathrm{LNH}$} \\
\hline OTU10 (Bifidobacterium pseudocatenulatum) & Low-Absent & 26.025 & 6.182 & 0.006 \\
\hline OTU10 (Bifidobacterium pseudocatenulatum) & Medium-Absent & 15.904 & 6.182 & 0.042 \\
\hline \multirow{2}{*}{ OTU10 (Bifidobacterium pseudocatenulatum) } & High—Absent & 42.040 & 8.094 & 0.002 \\
\hline & & \multicolumn{3}{|c|}{ FLNH } \\
\hline OTU20 (Bacteroides fragilis) & Low-Absent & -145.411 & 41.110 & 0.010 \\
\hline OTU20 (Bacteroides fragilis) & High-Absent & -191.839 & 68.517 & 0.027 \\
\hline \multirow[t]{2}{*}{ OTU29 (Enterococcus faecalis) } & Low-Absent & 194.456 & 83.620 & 0.049 \\
\hline & & \multicolumn{3}{|c|}{ DFLNH } \\
\hline OTU04 (Bifidobacterium breve) & Medium-Absent & 66.004 & 21.207 & 0.021 \\
\hline \multirow[t]{3}{*}{ OTU04 (Bifidobacterium breve) } & High-Absent & 65.513 & 16.197 & 0.007 \\
\hline & & \multicolumn{3}{|c|}{ Day 90} \\
\hline & & \multicolumn{3}{|c|}{$6^{\prime} \mathrm{SL}$} \\
\hline \multirow[t]{2}{*}{ OTU24 (Bifidobacterium longum) } & Low-Absent & -53.183 & 22.870 & 0.049 \\
\hline & & \multicolumn{3}{|c|}{ LNnT } \\
\hline OTU13 (Raoultella ornithinolytica) & Low-Absent & 139.726 & 45.827 & 0.019 \\
\hline \multirow[t]{2}{*}{ OTU29 (Enterococcus faecalis) } & Low-Absent & 141.980 & 42.530 & 0.010 \\
\hline & & \multicolumn{3}{|c|}{ LNFP III } \\
\hline OTU25 (Parabacteroides distasonis) & Low-Absent & 5.314 & 2.284 & 0.048 \\
\hline \multirow[t]{2}{*}{ OTU29 (Enterococcus faecalis) } & Low-Absent & 7.553 & 2.902 & 0.032 \\
\hline & & \multicolumn{3}{|c|}{ LSTc } \\
\hline \multirow[t]{2}{*}{ OTU03 (Bifidobacterium longum subsp. infantis) } & Low-Absent & 56.415 & 14.472 & 0.008 \\
\hline & & \multicolumn{3}{|c|}{ DFLNT } \\
\hline \multirow[t]{2}{*}{ OTU24 (Bifidobacterium longum) } & Low-Absent & -259.858 & 97.869 & 0.029 \\
\hline & & \multicolumn{3}{|c|}{ FLNH } \\
\hline OTU04 (Bifidobacterium breve) & High-Absent & -60.398 & 20.607 & 0.019 \\
\hline & & FDS & $\mathrm{NH}$ & \\
\hline OTU25 (Parabacteroides distasonis) & Low-Absent & 187.335 & 78.648 & 0.044 \\
\hline & & Day & & \\
\hline & & $2^{\prime}$ & & \\
\hline OTU05 (Streptococcus salivarius) & Low-Absent & 794.461 & 324.411 & 0.040 \\
\hline & & & & \\
\hline OTU05 (Streptococcus salivarius) & Low-Absent & -579.838 & 191.043 & 0.016 \\
\hline & & DF & & \\
\hline OTU24 (Bifidobacterium longum) & Low-Absent & -143.516 & 16.277 & $<0.001$ \\
\hline OTU25 (Parabacteroides distasonis) & Low-Absent & -130.102 & 56.234 & 0.049 \\
\hline
\end{tabular}


Table A5. Cont.

\begin{tabular}{|c|c|c|c|c|}
\hline \multirow{2}{*}{ Bacterial OTUs } & \multirow{2}{*}{$\begin{array}{c}\text { Comparison } \\
\text { (Parameter-Intercept) }\end{array}$} & Estimated Difference & SE & $p$-Value \\
\hline & & \multicolumn{3}{|c|}{ Day 120} \\
\hline & & \multicolumn{3}{|c|}{$3^{\prime} \mathrm{SL}$} \\
\hline \multirow[t]{2}{*}{ OTU29 (Enterococcus faecalis) } & Low-Absent & 113.907 & 12.184 & $<0.001$ \\
\hline & & \multicolumn{3}{|c|}{ LNT } \\
\hline OTU06 (Escherichia coli) & High-Absent & 425.170 & 137.772 & 0.022 \\
\hline \multirow[t]{2}{*}{ OTU25 (Parabacteroides distasonis) } & Low-Absent & 402.129 & 149.964 & 0.028 \\
\hline & & \multicolumn{3}{|c|}{ LNFP I } \\
\hline \multirow[t]{2}{*}{ OTU20 (Bacteroides fragilis) } & Low-Absent & 394.347 & 125.257 & 0.016 \\
\hline & & \multicolumn{3}{|c|}{ LNFP II } \\
\hline \multirow[t]{2}{*}{ OTU05 (Streptococcus salivarius) } & Low-Absent & -307.140 & 122.055 & 0.036 \\
\hline & & \multicolumn{3}{|c|}{$\mathrm{LSTb}$} \\
\hline \multirow[t]{2}{*}{ OTU06 (Escherichia coli) } & High-Absent & 37.797 & 14.733 & 0.043 \\
\hline & & \multicolumn{3}{|c|}{ DFLNT } \\
\hline \multirow[t]{2}{*}{ OTU06 (Escherichia coli) } & Medium-Absent & 358.632 & 107.565 & 0.016 \\
\hline & & \multicolumn{3}{|c|}{ LNH } \\
\hline \multirow[t]{2}{*}{ OTU20 (Bacteroides fragilis) } & Low-Absent & 47.773 & 7.970 & $<0.001$ \\
\hline & & \multicolumn{3}{|c|}{ FLNH } \\
\hline OTU06 (Escherichia coli) & High-Absent & 91.548 & 35.911 & 0.044 \\
\hline \multirow[t]{2}{*}{ OTU25 (Parabacteroides distasonis) } & Low-Absent & 105.044 & 39.804 & 0.030 \\
\hline & & \multicolumn{3}{|c|}{ DFLNH } \\
\hline \multirow[t]{2}{*}{ OTU14 (Veillonella nakazawae) } & Low-Absent & -43.460 & 16.298 & 0.032 \\
\hline & & \multicolumn{3}{|c|}{ FDSLNH } \\
\hline OTU24 (Bifidobacterium longum) & Low-Absent & 226.449 & 71.658 & 0.016 \\
\hline OTU25 (Parabacteroides distasonis) & Low-Absent & 317.661 & 94.893 & 0.010 \\
\hline
\end{tabular}

The OTUs (operational taxonomic unit) forming $>1 \%$ of relative abundance in infant oral samples were only included in the ANOVA analysis and the OTU relative abundance was categorized into four levels as high $=>30 \%$, medium $=5-30 \%$, low $=<5 \%$ and absent. Estimated difference represent $\mathrm{HMO}$ difference from the reference group. 2'FL-2'-fucosyllactose; 3'SL-3'-sialyllactose; 3FL-3-fucosyllactose; 6'SL-6'-sialyllactose; DFLacdifucosyllactose; DFLNH — difucosyllacto-N-hexaose; DFLNT—difucosyllacto-N-tetrose; DSLNH—disialyllactoN-hexaose; DSLNT_disialyllacto-N-tetraose; FDSLNH—fucodisialyllacto-N-hexaose; FLNH—fucosyllacto-Nhexaose; LNFP I-lacto-N-fucopentaose; LNFP II-lacto-N-fucopentaose II; LNFP III-lacto-N-fucopentaose; LNH-lacto-N-hexaose; LNnT-lacto-N-neotetraose; LNT-lacto-N-tetrose; LSTb-sialyl-lacto-N-tetraose b; LSTc-sialyl-lacto-N-tetraose c. 


\section{A) Maternal faecal}

M1

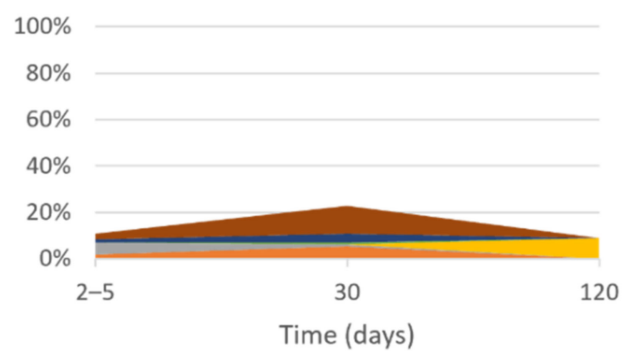

M3

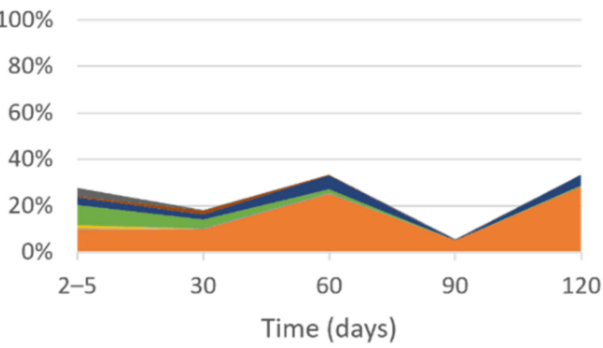

M5

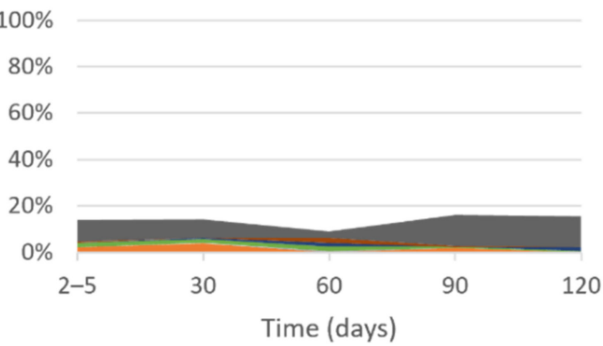

M7

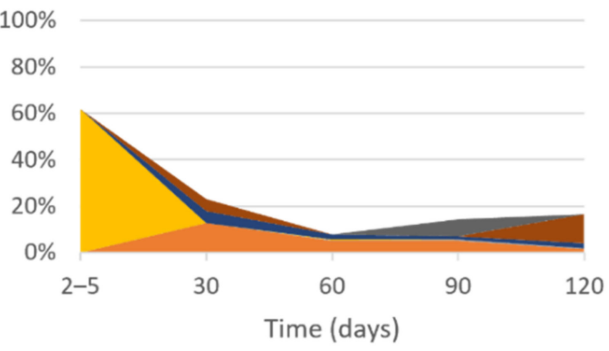

M9

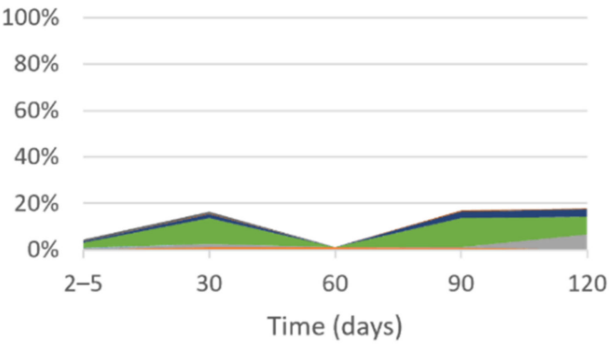

$\mathrm{M} 2$

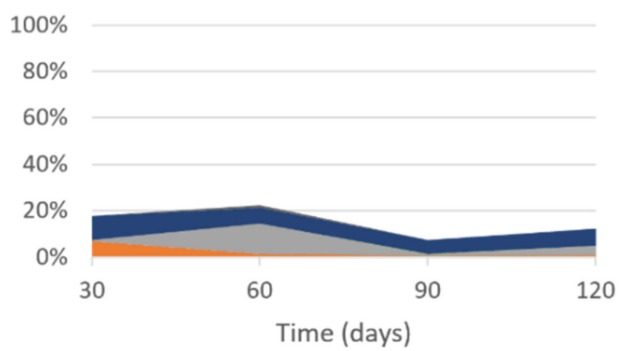

M4

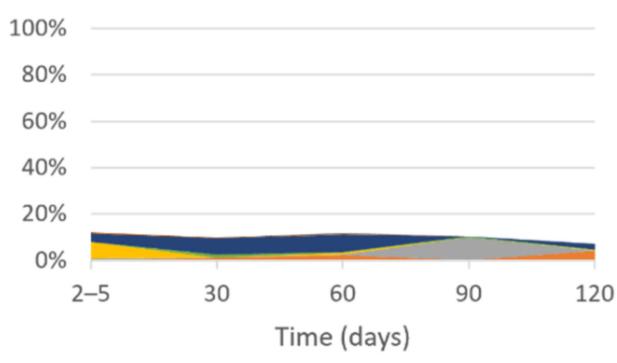

M6

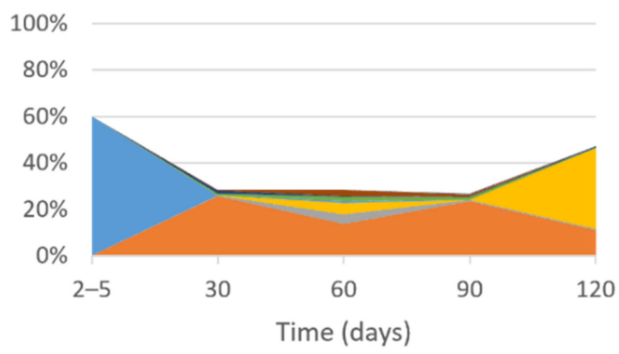

M8

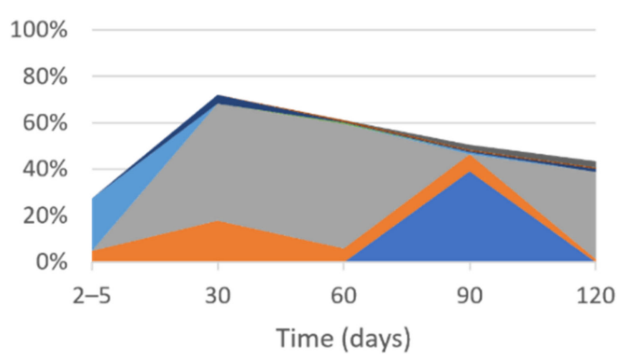

M10

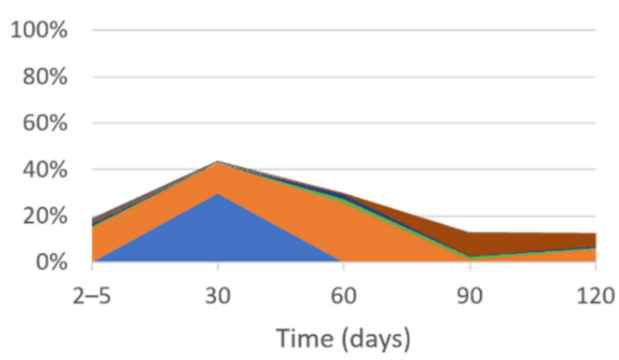

- OTU64 (Bifidobacterium adolescentis)

- OTU61 (Bacteroides uniformis)

- OTU43

(Faecalibacterium prausnitzii)

n OTU41 (Bacteroides dorei)

- OTU39 (Dialister invisus)

- OTU35

(Bacilli_c;RF39_o;RF39_f a;RF39_ge)

\section{= OTU27 (Akkermansia} muciniphila)

- OTU23 (Phocaeicola vulgatus)

- OTU06 (Escherichia coli)

Figure A1. Cont. 


\section{B) Human milk}

M1

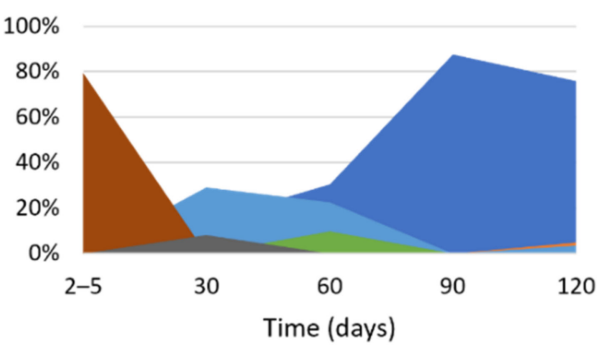

M3

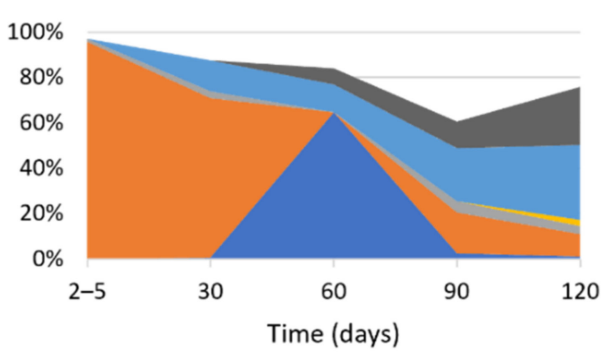

M5

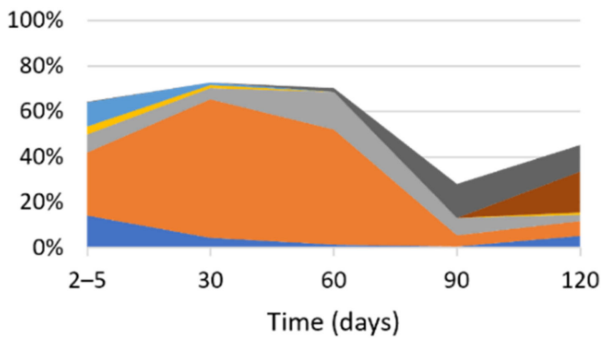

M7

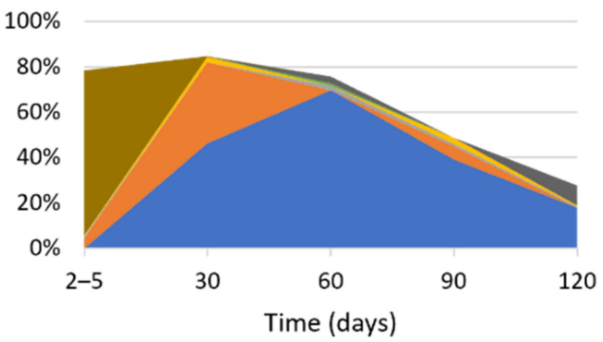

M9

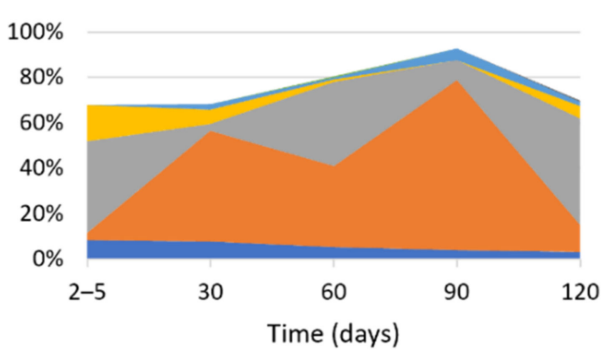

M2

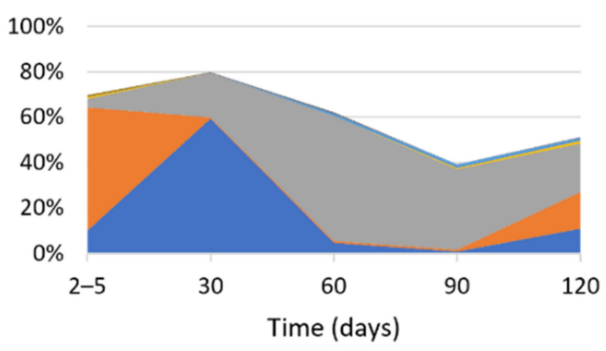

M4

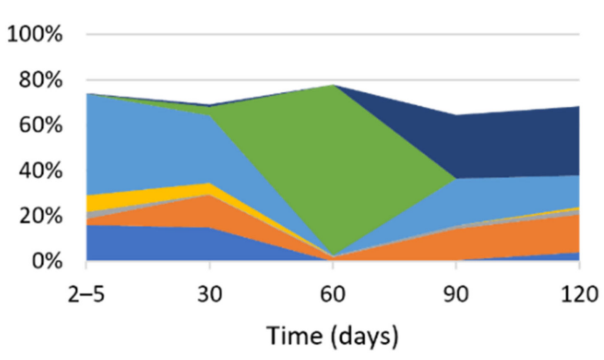

M6

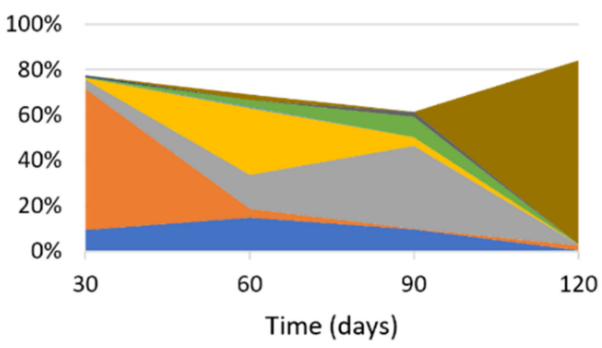

M8

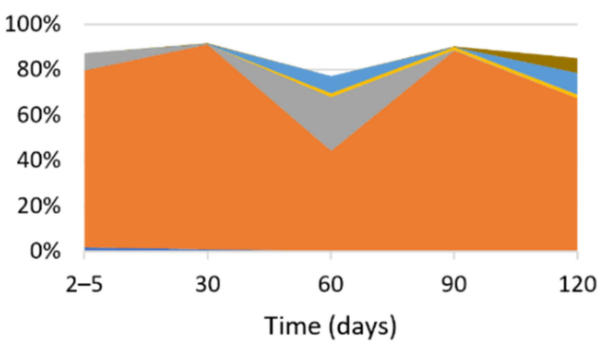

M10

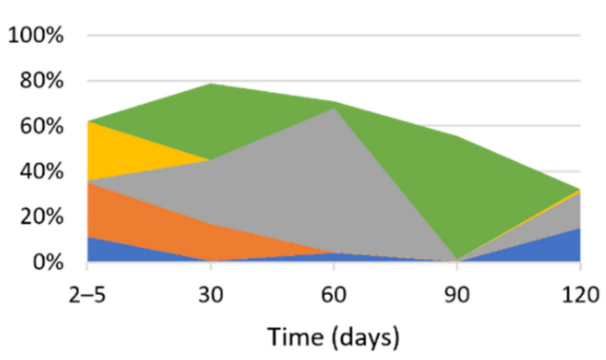

- OTU21 (Streptococcus agalactiae)

- OTU16 (Streptococcus lactarius)

- OTU15 (Moraxella osloensis)

- OTU12 (Streptococcus anginosus)

- OTU09 (Acinetobacter johnsonii)

- OTU08 (Cutibacterium acnes)

n OTU07 (Gemella haemolysans)

- OTU05 (Streptococcus salivarius)

nOTU02

(Staphylococcus epidermidis)

n OTU01 (Streptococcus mitis)

Figure A1. Cont. 


\section{C) Infant oral}

M1

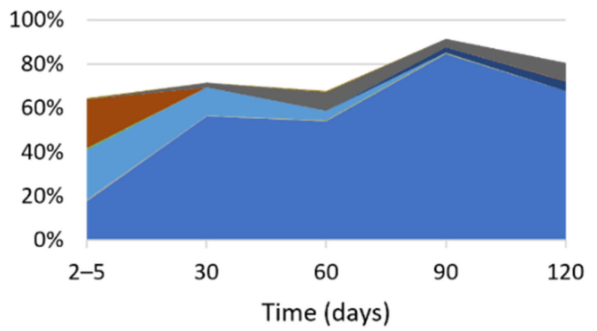

M3

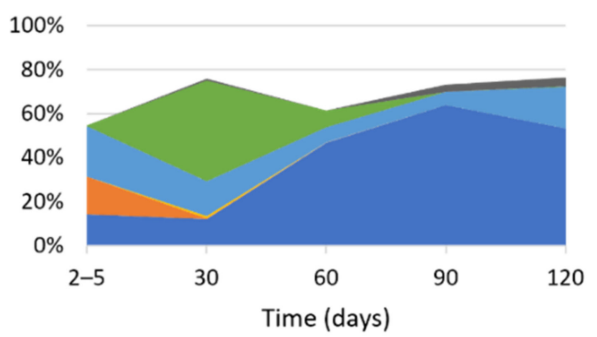

M5

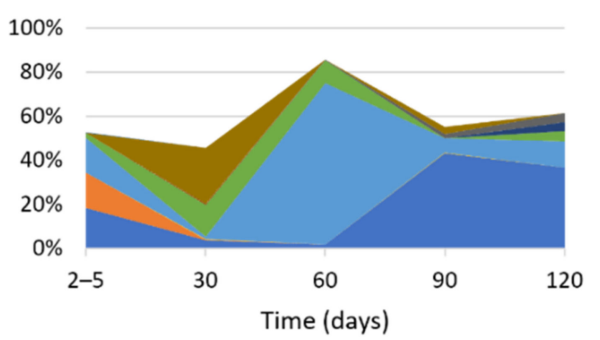

M7

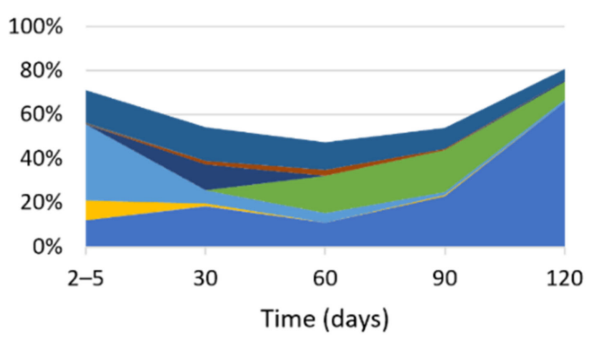

M9

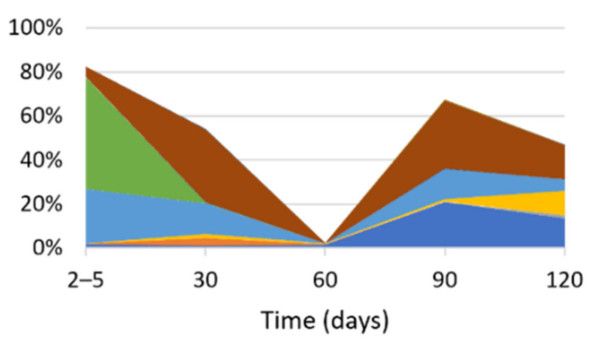

M2

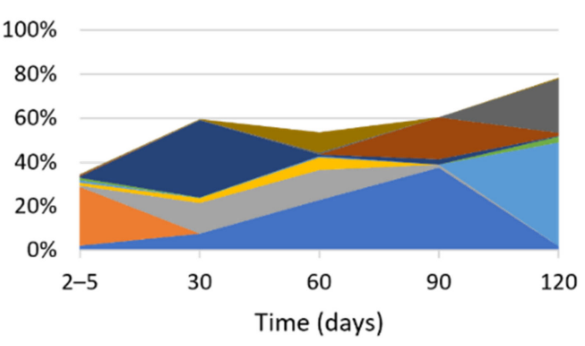

M4

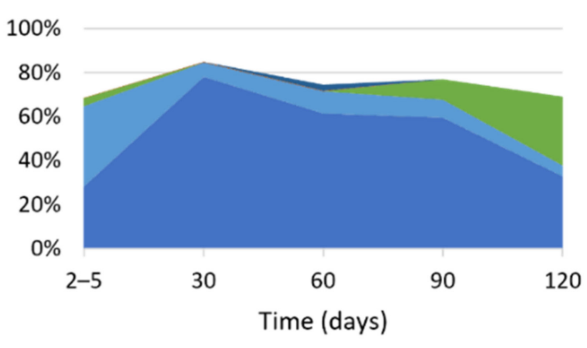

M6

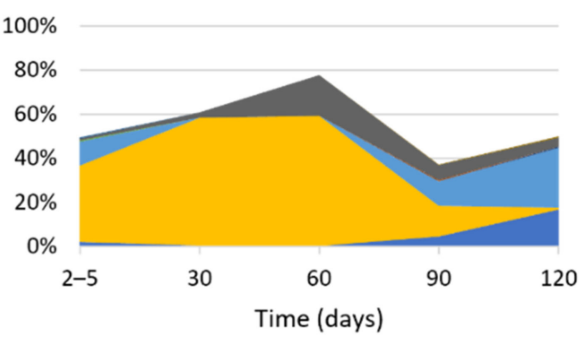

M8

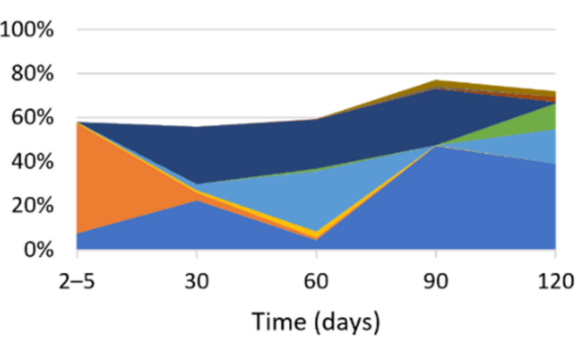

M10

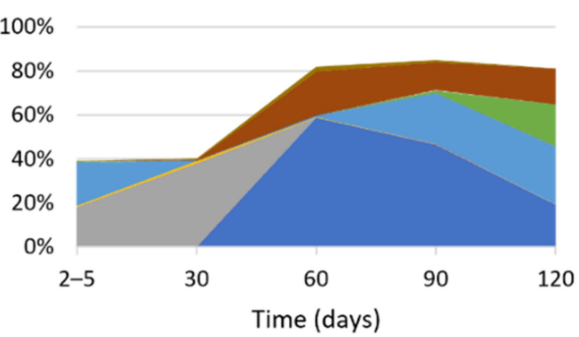

- OTU32 (Haemophilus parainfluenzae)

- OTU22 (Bergeyella sp.)

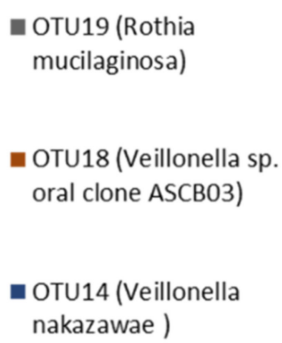

OTU11 (Haemophilus haemolyticus)

- OTU07 (Gemella haemolysans)

OTU05 (Streptococcus salivarius)

= OTU03

(Bifidobacterium longum subsp. infantis )

n OTU02

(Staphylococcus epidermidis)

n OTU01 (Streptococcus mitis)

Figure A1. Cont. 


\section{D) Infant faecal}

M1

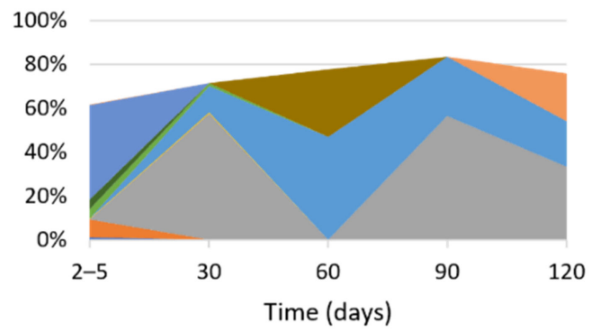

M3

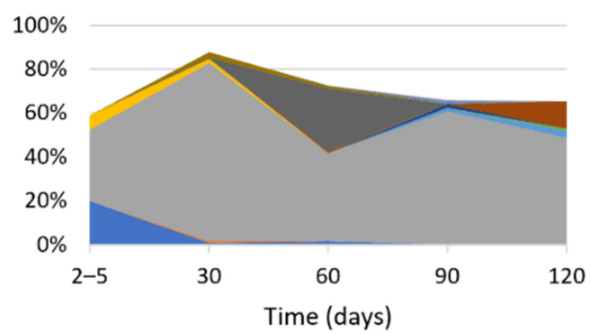

M5

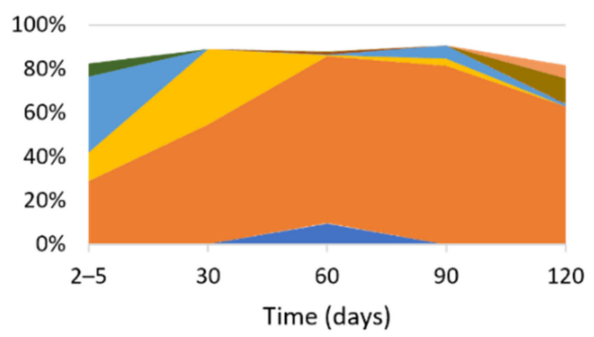

M7

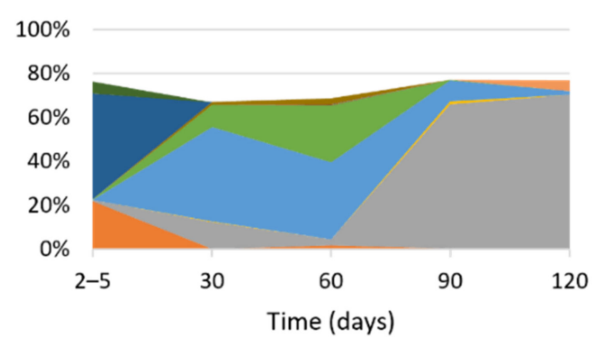

M9

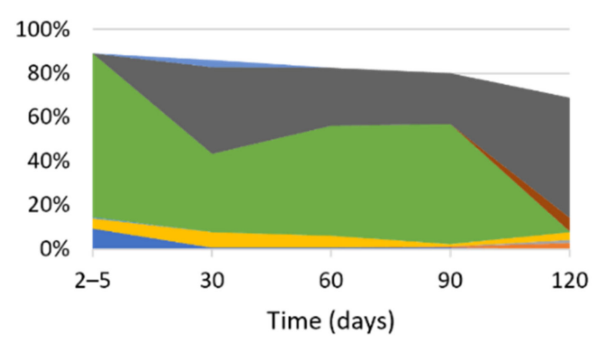

M2

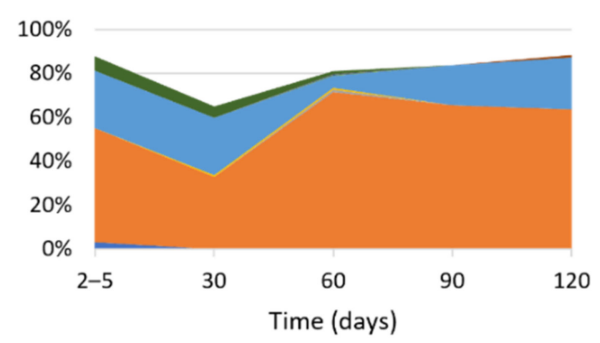

M4

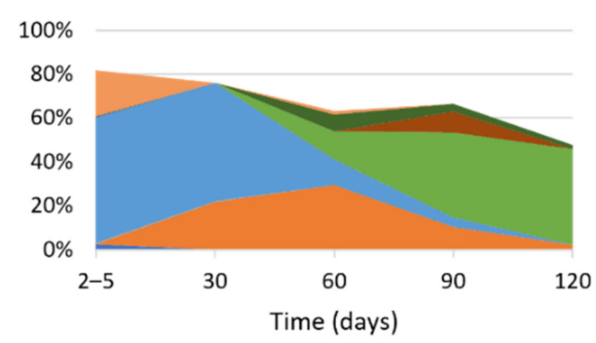

M6

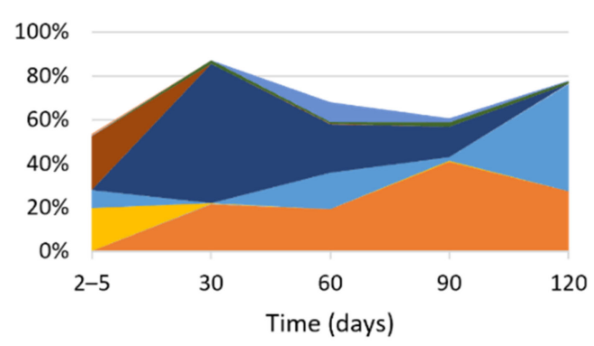

M8

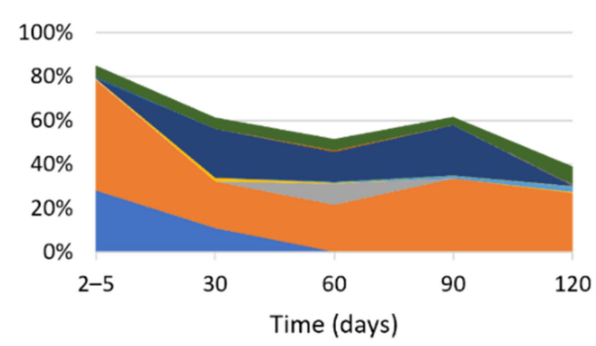

M10

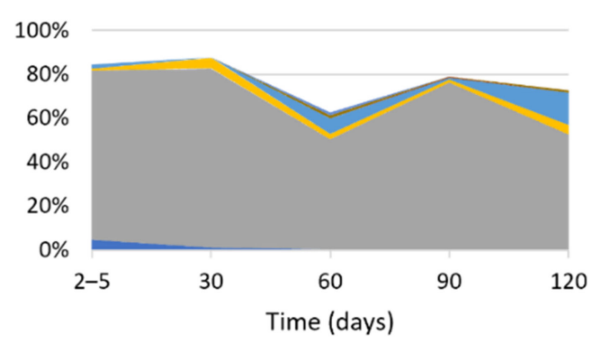

- OTU29 (Enterococcus faecalis)

- OTU25 (Parabacteroides distasonis)

- OTU24 (Bifidobacterium longum)

- OTU21 (Streptococcus agalactiae)

- OTU20 (Bacteroides

fragilis)

- OTU17 (Klebsiella pneumoniae)

- OTU14 (Veillonella nakazawae)

- OTU13 (Raoultella ornithinolytica)

- OTU10 (Bifidobacterium pseudocatenulatum)

OTU06 (Escherichia coli)

- OTU05 (Streptococcus salivarius)

- OTU04 (Bifidobacterium breve )

- ОTU03 (Bifidobacterium longum subsp. infantis )

- OTU02 (Staphylococcus epidermidis)

Figure A1. Individual variation in microbiome composition over time. (A) Maternal faecal, (B) Human milk, (C) Infant oral and (D) Infant faecal samples. Relative abundance is displayed on the y-axis. Missing maternal faecal samples: M1 days 30 and 60, M2 day 2-5. Missing HM sample: M6 day $2-5$. 


\section{References}

1. Stinson, L.F. Establishment of the early-life microbiome: A DOHaD perspective. J. Dev. Orig. Health Dis. 2020, 11, 201-210. [CrossRef]

2. Stiemsma, L.T.; Michels, K.B. The role of the microbiome in the developmental origins of health and disease. Pediatrics 2018, 141, e20172437. [CrossRef] [PubMed]

3. Stanislawski, M.A.; Dabelea, D.; Wagner, B.D.; Iszatt, N.; Dahl, C.; Sontag, M.K.; Knight, R.; Lozupone, C.A.; Eggesbo, M. Gut microbiota in the first 2 years of life and the association with body mass index at age 12 in a norwegian birth cohort. $m$ Bio 2018, 9, e01751-18. [CrossRef]

4. Kostic, A.D.; Gevers, D.; Siljander, H.; Vatanen, T.; Hyotylainen, T.; Hamalainen, A.M.; Peet, A.; Tillmann, V.; Poho, P.; Mattila, I.; et al. The dynamics of the human infant gut microbiome in development and in progression toward type 1 diabetes. Cell Host Microbe 2015, 17, 260-273. [CrossRef]

5. Azad, M.B.; Konya, T.; Guttman, D.S.; Field, C.J.; Sears, M.R.; HayGlass, K.T.; Mandhane, P.J.; Turvey, S.E.; Subbarao, P.; Becker, A.B.; et al. Infant gut microbiota and food sensitization: Associations in the first year of life. Clin. Exp. Allergy 2015, 45, 632-643. [CrossRef] [PubMed]

6. Arrieta, M.C.; Stiemsma, L.T.; Dimitriu, P.A.; Thorson, L.; Russell, S.; Yurist-Doutsch, S.; Kuzeljevic, B.; Gold, M.J.; Britton, H.M.; Lefebvre, D.L.; et al. Early infancy microbial and metabolic alterations affect risk of childhood asthma. Sci. Transl. Med. 2015, 7, 307ra152. [CrossRef] [PubMed]

7. Mohajeri, M.H.; La Fata, G.; Steinert, R.E.; Weber, P. Relationship between the gut microbiome and brain function. Nutr. Rev. 2018, 76, 481-496. [CrossRef] [PubMed]

8. Van den Elsen, L.W.J.; Garssen, J.; Burcelin, R.; Verhasselt, V. Shaping the gut microbiota by breastfeeding: The gateway to allergy prevention? Front. Pediatr. 2019, 7, 47. [CrossRef]

9. Stewart, C.J.; Ajami, N.J.; O’Brien, J.L.; Hutchinson, D.S.; Smith, D.P.; Wong, M.C.; Ross, M.C.; Lloyd, R.E.; Doddapaneni, H.; Metcalf, G.A.; et al. Temporal development of the gut microbiome in early childhood from the TEDDY study. Nature 2018, 562, 583-588. [CrossRef]

10. Roswall, J.; Olsson, L.M.; Kovatcheva-Datchary, P.; Nilsson, S.; Tremaroli, V.; Simon, M.-C.; Kiilerich, P.; Akrami, R.; Krämer, M.; Uhlén, M.; et al. Developmental trajectory of the healthy human gut microbiota during the first 5 years of life. Cell Host Microbe 2021, 29, 765-776.e763. [CrossRef]

11. Nagpal, R.; Tsuji, H.; Takahashi, T.; Nomoto, K.; Kawashima, K.; Nagata, S.; Yamashiro, Y. Ontogenesis of the gut microbiota composition in healthy, full-term, vaginally born and breast-fed infants over the first 3 years of life: A quantitative bird's-eye view. Front. Microbiol. 2017, 8, 1388. [CrossRef] [PubMed]

12. Yassour, M.; Jason, E.; Hogstrom, L.J.; Arthur, T.D.; Tripathi, S.; Siljander, H.; Selvenius, J.; Oikarinen, S.; Hyoty, H.; Virtanen, S.M.; et al. Strain-level analysis of mother-to-child bacterial transmission during the first few months of life. Cell Host Microbe 2018, 24, 146-154.e144. [CrossRef] [PubMed]

13. Ferretti, P.; Pasolli, E.; Tett, A.; Asnicar, F.; Gorfer, V.; Fedi, S.; Armanini, F.; Truong, D.T.; Manara, S.; Zolfo, M.; et al. Mother-toinfant microbial transmission from different body sites shapes the developing infant gut microbiome. Cell Host Microbe 2018, 24, 133-145.e135. [CrossRef] [PubMed]

14. Murphy, K.; Curley, D.; O'Callaghan, T.F.; O'Shea, C.A.; Dempsey, E.M.; O’Toole, P.W.; Ross, R.P.; Ryan, C.A.; Stanton, C. The composition of human milk and infant faecal microbiota over the first three months of life: A pilot study. Sci. Rep. 2017, 7, 40597. [CrossRef]

15. Drell, T.; Stsepetova, J.; Simm, J.; Rull, K.; Aleksejeva, A.; Antson, A.; Tillmann, V.; Metsis, M.; Sepp, E.; Salumets, A.; et al. The Influence of different maternal microbial communities on the development of infant gut and oral microbiota. Sci. Rep. 2017, 7, 9940. [CrossRef]

16. Tanaka, M.; Nakayama, J. Development of the gut microbiota in infancy and its impact on health in later life. Allergol. Int. 2017, 66, 515-522. [CrossRef]

17. Valles, Y.; Artacho, A.; Pascual-Garcia, A.; Ferrus, M.L.; Gosalbes, M.J.; Abellan, J.J.; Francino, M.P. Microbial succession in the gut: Directional trends of taxonomic and functional change in a birth cohort of Spanish infants. PLoS Genet. 2014, 10, e1004406. [CrossRef]

18. Lackey, K.A.; Williams, J.E.; Meehan, C.L.; Zachek, J.A.; Benda, E.D.; Price, W.J.; Foster, J.A.; Sellen, D.W.; Kamau-Mbuthia, E.W.; Kamundia, E.W.; et al. What's normal? microbiomes in human milk and infant feces are related to each other but vary geographically: The INSPIRE study. Front. Nutr. 2019, 6, 45. [CrossRef]

19. Drago, L.; Toscano, M.; De Grandi, R.; Grossi, E.; Padovani, E.M.; Peroni, D.G. Microbiota network and mathematic microbe mutualism in colostrum and mature milk collected in two different geographic areas: Italy versus Burundi. ISME J. 2017, 11, 875-884. [CrossRef]

20. McGuire, M.K.; Meehan, C.L.; McGuire, M.A.; Williams, J.E.; Foster, J.; Sellen, D.W.; Kamau-Mbuthia, E.W.; Kamundia, E.W.; Mbugua, S.; Moore, S.E.; et al. What's normal? oligosaccharide concentrations and profiles in milk produced by healthy women vary geographically. Am. J. Clin. Nutr. 2017, 105, 1086-1100. [CrossRef]

21. Ding, M.; Qi, C.; Yang, Z.; Jiang, S.; Bi, Y.; Lai, J.; Sun, J. Geographical location specific composition of cultured microbiota and Lactobacillus occurrence in human breast milk in China. Food Funct. 2019, 10, 554-564. [CrossRef] 
22. Biagi, E.; Quercia, S.; Aceti, A.; Beghetti, I.; Rampelli, S.; Turroni, S.; Faldella, G.; Candela, M.; Brigidi, P.; Corvaglia, L. The bacterial ecosystem of mother's milk and infant's mouth and gut. Front. Microbiol. 2017, 8, 1214. [CrossRef] [PubMed]

23. Costello, E.K.; Carlisle, E.M.; Bik, E.M.; Morowitz, M.J.; Relman, D.A. Microbiome assembly across multiple body sites in low-birthweight infants. mBio 2013, 4, e00782-13. [CrossRef] [PubMed]

24. Williams, J.E.; Carrothers, J.M.; Lackey, K.A.; Beatty, N.F.; Brooker, S.L.; Peterson, H.K.; Steinkamp, K.M.; York, M.A.; Shafii, B.; Price, W.J.; et al. Strong multivariate relations exist among milk, oral, and fecal microbiomes in mother-infant dyads during the first six months postpartum. J. Nutr. 2019, 149, 902-914. [CrossRef] [PubMed]

25. Ling, Z.; Kong, J.; Jia, P.; Wei, C.; Wang, Y.; Pan, Z.; Huang, W.; Li, L.; Chen, H.; Xiang, C. Analysis of oral microbiota in children with dental caries by PCR-DGGE and barcoded pyrosequencing. Microb. Ecol. 2010, 60, 677-690. [CrossRef]

26. Chen, C.; Hemme, C.; Beleno, J.; Shi, Z.J.; Ning, D.; Qin, Y.; Tu, Q.; Jorgensen, M.; He, Z.; Wu, L.; et al. Oral microbiota of periodontal health and disease and their changes after nonsurgical periodontal therapy. ISME J. 2018, 12, 1210-1224. [CrossRef]

27. Sulyanto, R.M.; Thompson, Z.A.; Beall, C.J.; Leys, E.J.; Griffen, A.L. The predominant oral microbiota is acquired early in an organized pattern. Sci. Rep. 2019, 9, 10550. [CrossRef]

28. Oba, P.M.; Holscher, H.D.; Mathai, R.A.; Kim, J.; Swanson, K.S. Diet influences the oral microbiota of infants during the first six months of life. Nutrients 2020, 12, 3400. [CrossRef]

29. Timby, N.; Domellof, M.; Holgerson, P.L.; West, C.E.; Lonnerdal, B.; Hernell, O.; Johansson, I. Oral microbiota in infants fed a formula supplemented with bovine milk fat globule membranes-A randomized controlled trial. PLoS ONE 2017, 12, e0169831. [CrossRef]

30. Mason, M.R.; Chambers, S.; Dabdoub, S.M.; Thikkurissy, S.; Kumar, P.S. Characterizing oral microbial communities across dentition states and colonization niches. Microbiome 2018, 6, 67. [CrossRef]

31. Dzidic, M.; Collado, M.C.; Abrahamsson, T.; Artacho, A.; Stensson, M.; Jenmalm, M.C.; Mira, A. Oral microbiome development during childhood: An ecological succession influenced by postnatal factors and associated with tooth decay. ISME J. 2018, 12, 2292-2306. [CrossRef] [PubMed]

32. Matsuyama, M.; Gomez-Arango, L.F.; Fukuma, N.M.; Morrison, M.; Davies, P.S.W.; Hill, R.J. Breastfeeding: A key modulator of gut microbiota characteristics in late infancy. J. Dev. Orig. Health Dis. 2019, 10, 206-213. [CrossRef] [PubMed]

33. Walsh, C.; Lane, J.A.; van Sinderen, D.; Hickey, R.M. Human milk oligosaccharides: Shaping the infant gut microbiota and supporting health. J. Funct. Foods 2020, 72, 104074. [CrossRef]

34. Asnicar, F.; Manara, S.; Zolfo, M.; Truong, D.T.; Scholz, M.; Armanini, F.; Ferretti, P.; Gorfer, V.; Pedrotti, A.; Tett, A.; et al. Studying vertical microbiome transmission from mothers to infants by strain-level metagenomic profiling. mSystems 2017, 2, e00164-16. [CrossRef] [PubMed]

35. Milani, C.; Mancabelli, L.; Lugli, G.A.; Duranti, S.; Turroni, F.; Ferrario, C.; Mangifesta, M.; Viappiani, A.; Ferretti, P.; Gorfer, V.; et al. Exploring vertical transmission of Bifidobacteria from mother to child. Appl. Environ. Microbiol. 2015, 81, 7078-7087. [CrossRef]

36. Jost, T.; Lacroix, C.; Braegger, C.P.; Rochat, F.; Chassard, C. Vertical mother-neonate transfer of maternal gut bacteria via breastfeeding. Environ. Microbiol. 2014, 16, 2891-2904. [CrossRef]

37. Duranti, S.; Lugli, G.A.; Mancabelli, L.; Armanini, F.; Turroni, F.; James, K.; Ferretti, P.; Gorfer, V.; Ferrario, C.; Milani, C.; et al. Maternal inheritance of bifidobacterial communities and bifidophages in infants through vertical transmission. Microbiome 2017, 5, 66. [CrossRef]

38. Koren, O.; Goodrich, J.K.; Cullender, T.C.; Spor, A.; Laitinen, K.; Backhed, H.K.; Gonzalez, A.; Werner, J.J.; Angenent, L.T.; Knight, R.; et al. Host remodeling of the gut microbiome and metabolic changes during pregnancy. Cell 2012, 150, 470-480. [CrossRef]

39. Carrothers, J.M.; York, M.A.; Brooker, S.L.; Lackey, K.A.; Williams, J.E.; Shafii, B.; Price, W.J.; Settles, M.L.; McGuire, M.A.; McGuire, M.K. Fecal microbial community structure is stable over time and related to variation in macronutrient and micronutrient intakes in lactating women. J. Nutr. 2015, 145, 2379-2388. [CrossRef]

40. Canul-Medina, G.; Fernandez-Mejia, C. Morphological, hormonal, and molecular changes in different maternal tissues during lactation and post-lactation. J. Physiol. Sci. 2019, 69, 825-835. [CrossRef]

41. Hunt, K.M.; Foster, J.A.; Forney, L.J.; Schutte, U.M.; Beck, D.L.; Abdo, Z.; Fox, L.K.; Williams, J.E.; McGuire, M.K.; McGuire, M.A. Characterization of the diversity and temporal stability of bacterial communities in human milk. PLoS ONE 2011, 6, e21313. [CrossRef] [PubMed]

42. Moossavi, S.; Sepehri, S.; Robertson, B.; Bode, L.; Goruk, S.; Field, C.J.; Lix, L.M.; de Souza, R.J.; Becker, A.B.; Mandhane, P.J.; et al. Composition and variation of the human milk microbiota are influenced by maternal and early-life factors. Cell Host Microbe 2019, 25, 324-335.e324. [CrossRef] [PubMed]

43. Hermansson, H.; Kumar, H.; Collado, M.C.; Salminen, S.; Isolauri, E.; Rautava, S. Breast milk microbiota Is shaped by mode of delivery and intrapartum antibiotic exposure. Front. Nutr. 2019, 6, 4. [CrossRef]

44. Wan, Y.; Jiang, J.; Lu, M.; Tong, W.; Zhou, R.; Li, J.; Yuan, J.; Wang, F.; Li, D. Human milk microbiota development during lactation and its relation to maternal geographic location and gestational hypertensive status. Gut Microbes 2020, 11, 1438-1449. [CrossRef]

45. Khodayar-Pardo, P.; Mira-Pascual, L.; Collado, M.C.; Martinez-Costa, C. Impact of lactation stage, gestational age and mode of delivery on breast milk microbiota. J. Perinatol. 2014, 34, 599-605. [CrossRef] [PubMed] 
46. Williams, J.E.; Carrothers, J.M.; Lackey, K.A.; Beatty, N.F.; York, M.A.; Brooker, S.L.; Shafii, B.; Price, W.J.; Settles, M.L.; McGuire, M.A.; et al. Human milk mcrobial community structure is relatively stable and related to variations in macronutrient and micronutrient intakes in healthy lactating women. J. Nutr. 2017, 147, 1739-1748. [CrossRef] [PubMed]

47. Cabrera-Rubio, R.; Collado, M.C.; Laitinen, K.; Salminen, S.; Isolauri, E.; Mira, A. The human milk microbiome changes over lactation and is shaped by maternal weight and mode of delivery. Am. J. Clin. Nutr. 2012, 96, 544-551. [CrossRef]

48. Li, S.W.; Watanabe, K.; Hsu, C.C.; Chao, S.H.; Yang, Z.H.; Lin, Y.J.; Chen, C.C.; Cao, Y.M.; Huang, H.C.; Chang, C.H.; et al. Bacterial composition and diversity in breast milk samples from mothers living in Taiwan and Mainland China. Front. Microbiol. 2017, 8, 965. [CrossRef]

49. Moossavi, S.; Miliku, K.; Sepehri, S.; Khafipour, E.; Azad, M.B. The prebiotic and probiotic properties of human milk: Implications for infant immune development and pediatric asthma. Front. Pediatr. 2018, 6, 197. [CrossRef]

50. Sela, D.A.; Mills, D.A. Nursing our microbiota: Molecular linkages between bifidobacteria and milk oligosaccharides. Trends Microbiol. 2010, 18, 298-307. [CrossRef]

51. Sela, D.A.; Chapman, J.; Adeuya, A.; Kim, J.H.; Chen, F.; Whitehead, T.R.; Lapidus, A.; Rokhsar, D.S.; Lebrilla, C.B.; German, J.B.; et al. The genome sequence of Bifidobacterium longum subsp. infantis reveals adaptations for milk utilization within the infant microbiome. Proc. Natl. Acad. Sci. USA 2008, 105, 18964-18969. [CrossRef] [PubMed]

52. Marcobal, A.; Barboza, M.; Froehlich, J.W.; Block, D.E.; German, J.B.; Lebrilla, C.B.; Mills, D.A. Consumption of human milk oligosaccharides by gut-related microbes. J. Agric. Food Chem. 2010, 58, 5334-5340. [CrossRef] [PubMed]

53. Pace, R.M.; Williams, J.E.; Robertson, B.; Lackey, K.A.; Meehan, C.L.; Price, W.J.; Foster, J.A.; Sellen, D.W.; Kamau-Mbuthia, E.W.; Kamundia, E.W.; et al. Variation in human milk compostion is related to differeces in milk and infant fecal microbial communities. Microorganisms 2021, 9, 1153. [CrossRef] [PubMed]

54. Wang, M.; Li, M.; Wu, S.; Lebrilla, C.B.; Chapkin, R.S.; Ivanov, I.; Donovan, S.M. Fecal microbiota composition of breast-fed infants is correlated with human milk oligosaccharides consumed. J. Pediatr. Gastroenterol. Nutr. 2015, 60, 825-833. [CrossRef]

55. Laursen, M.F.; Pekmez, C.T.; Larsson, M.W.; Lind, M.V.; Yonemitsu, C.; Larnkjær, A.; Mølgaard, C.; Bode, L.; Dragsted, L.O.; Michaelsen, K.F.; et al. Maternal milk microbiota and oligosaccharides contribute to the infant gut microbiota assembly. ISME Commun. 2021, 1, 21. [CrossRef]

56. Ramani, S.; Stewart, C.J.; Laucirica, D.R.; Ajami, N.J.; Robertson, B.; Autran, C.A.; Shinge, D.; Rani, S.; Anandan, S.; Hu, L.; et al. Human milk oligosaccharides, milk microbiome and infant gut microbiome modulate neonatal rotavirus infection. Nat. Commun. 2018, 9, 5010. [CrossRef]

57. De Leoz, M.L.; Kalanetra, K.M.; Bokulich, N.A.; Strum, J.S.; Underwood, M.A.; German, J.B.; Mills, D.A.; Lebrilla, C.B. Human milk glycomics and gut microbial genomics in infant feces show a correlation between human milk oligosaccharides and gut microbiota: A proof-of-concept study. J. Proteome Res. 2015, 14, 491-502. [CrossRef] [PubMed]

58. Crusell, M.K.W.; Hansen, T.H.; Nielsen, T.; Allin, K.H.; Ruhlemann, M.C.; Damm, P.; Vestergaard, H.; Rorbye, C.; Jorgensen, N.R.; Christiansen, O.B.; et al. Gestational diabetes is associated with change in the gut microbiota composition in third trimester of pregnancy and postpartum. Microbiome 2018, 6, 89. [CrossRef] [PubMed]

59. Wang, J.; Zheng, J.; Shi, W.; Du, N.; Xu, X.; Zhang, Y.; Ji, P.; Zhang, F.; Jia, Z.; Wang, Y.; et al. Dysbiosis of maternal and neonatal microbiota associated with gestational diabetes mellitus. Gut 2018, 67, 1614-1625. [CrossRef] [PubMed]

60. Maher, S.E.; O’Brien, E.C.; Moore, R.L.; Byrne, D.F.; Geraghty, A.A.; Saldova, R.; Murphy, E.F.; Van Sinderen, D.; Cotter, P.D.; McAuliffe, F.M. The association between the maternal diet and the maternal and infant gut microbiome: A systematic review. Br. J. Nutr. 2020, 1-29. [CrossRef]

61. Keohane, D.M.; Ghosh, T.S.; Jeffery, I.B.; Molloy, M.G.; O’Toole, P.W.; Shanahan, F. Microbiome and health implications for ethnic minorities after enforced lifestyle changes. Nat. Med. 2020, 26, 1089-1095. [CrossRef] [PubMed]

62. Durazzi, F.; Sala, C.; Castellani, G.; Manfreda, G.; Remondini, D.; De Cesare, A. Comparison between 16S rRNA and shotgun sequencing data for the taxonomic characterization of the gut microbiota. Sci. Rep. 2021, 11, 3030. [CrossRef] [PubMed]

63. Barrett, H.L.; Gomez-Arango, L.F.; Wilkinson, S.A.; McIntyre, H.D.; Callaway, L.K.; Morrison, M.; Dekker Nitert, M. A vegetarian diet is a major determinant of gut microbiota composition in early pregnancy. Nutrients 2018, 10, 890. [CrossRef]

64. Mandal, S.; Godfrey, K.M.; McDonald, D.; Treuren, W.V.; Bjørnholt, J.V.; Midtvedt, T.; Moen, B.; Rudi, K.; Knight, R.; Brantsæter, A.L.; et al. Fat and vitamin intakes during pregnancy have stronger relations with a pro-inflammatory maternal microbiota than does carbohydrate intake. Microbiome 2016, 4, 55. [CrossRef] [PubMed]

65. Roytio, H.; Mokkala, K.; Vahlberg, T.; Laitinen, K. Dietary intake of fat and fibre according to reference values relates to higher gut microbiota richness in overweight pregnant women. Br. J. Nutr. 2017, 118, 343-352. [CrossRef] [PubMed]

66. Kota, S.K.; Gayatri, K.; Jammula, S.; Kota, S.K.; Krishna, S.V.; Meher, L.K.; Modi, K.D. Endocrinology of parturition. Indian J. Endocrinol. Metab. 2013, 17, 50-59. [CrossRef] [PubMed]

67. Edwards, S.M.; Cunningham, S.A.; Dunlop, A.L.; Corwin, E.J. The maternal gut microbiome during pregnancy. MCN Am. J. Matern. Child Nurs. 2017, 42, 310-317. [CrossRef]

68. Grajeda, R.; Perez-Escamilla, R. Stress during labor and delivery is associated with delayed onset of lactation among urban Guatemalan women. J. Nutr. 2002, 132, 3055-3060. [CrossRef]

69. Ilchmann-Diounou, H.; Menard, S. Psychological stress, intestinal barrier dysfunctions, and autoimmune disorders: An overview. Front. Immunol. 2020, 11, 1823. [CrossRef] 
70. Ward, T.L.; Hosid, S.; Ioshikhes, I.; Altosaar, I. Human milk metagenome: A functional capacity analysis. BMC Microbiol. 2013, 13, 116. [CrossRef]

71. Jimenez, E.; de Andres, J.; Manrique, M.; Pareja-Tobes, P.; Tobes, R.; Martinez-Blanch, J.F.; Codoner, F.M.; Ramon, D.; Fernandez, L.; Rodriguez, J.M. Metagenomic analysis of milk of healthy and mastitis-suffering women. J. Hum. Lact. 2015, 31, 406-415. [CrossRef]

72. Grice, E.A.; Kong, H.H.; Conlan, S.; Deming, C.B.; Davis, J.; Young, A.C.; Program, N.C.S.; Bouffard, G.G.; Blakesley, R.W.; Murray, P.R.; et al. Topographical and temporal diversity of the human skin microbiome. Science 2009, 324, 1190-1192. [CrossRef] [PubMed]

73. Otto, M. Staphylococcus epidermidis_The 'accidental' pathogen. Nat. Rev. Microbiol. 2009, 7, 555-567. [CrossRef] [PubMed]

74. Moles, L.; Gomez, M.; Moroder, E.; Bustos, G.; Melgar, A.; Del Campo, R.; Rodriguez, J.M. Staphylococcus epidermidis in feedings and feces of preterm neonates. PLoS ONE 2020, 15, e0227823. [CrossRef]

75. Dong, Y.; Speer, C.P. The role of Staphylococcus epidermidis in neonatal sepsis: Guarding angel or pathogenic devil? Int. J. Med. Microbiol. 2014, 304, 513-520. [CrossRef] [PubMed]

76. Soeorg, H.; Metsvaht, T.; Eelmae, I.; Merila, M.; Treumuth, S.; Huik, K.; Jurna-Ellam, M.; Ilmoja, M.L.; Lutsar, I. The role of breast milk in the colonization of neonatal gut and skin with coagulase-negative staphylococci. Pediatr. Res. 2017, 82, 759-767. [CrossRef]

77. Boix-Amoros, A.; Collado, M.C.; Mira, A. Relationship between milk microbiota, bacterial load, macronutrients, and human cells during lactation. Front. Microbiol. 2016, 7, 492. [CrossRef] [PubMed]

78. Craig, S.J.; Blankenberg, D.; Parodi, A.C.L.; Paul, I.M.; Birch, L.L.; Savage, J.S.; Marini, M.E.; Stokes, J.L.; Nekrutenko, A.; Reimherr, M. Child weight gain trajectories linked to oral microbiota composition. Sci. Rep. 2018, 8, 14030. [CrossRef] [PubMed]

79. Xiao, J.; Fiscella, K.A.; Gill, S.R. Oral microbiome: Possible harbinger for children's health. Int. J. Oral Sci. 2020, 12, 12. [CrossRef]

80. Reddy, R.M.; Weir, W.B.; Barnett, S.; Heiden, B.T.; Orringer, M.B.; Lin, J.; Chang, A.C.; Carrott, P.W.; Lynch, W.R.; Beer, D.G.; et al. Increased variance in oral and gastric microbiome correlates with esophagectomy anastomotic leak. Ann. Thorac. Surg. 2018, 105, 865-870. [CrossRef]

81. Blod, C.; Schlichting, N.; Schulin, S.; Suttkus, A.; Peukert, N.; Stingu, C.S.; Hirsch, C.; Elger, W.; Lacher, M.; Buhligen, U.; et al. The oral microbiome-the relevant reservoir for acute pediatric appendicitis? Int. J. Colorectal Dis. 2018, 33, 209-218. [CrossRef]

82. Premaraj, T.S.; Vella, R.; Chung, J.; Lin, Q.; Panier, H.; Underwood, K.; Premaraj, S.; Zhou, Y. Ethnic variation of oral microbiota in children. Sci. Rep. 2020, 10, 14788. [CrossRef] [PubMed]

83. Li, J.; Quinque, D.; Horz, H.P.; Li, M.; Rzhetskaya, M.; Raff, J.A.; Hayes, M.G.; Stoneking, M. Comparative analysis of the human saliva microbiome from different climate zones: Alaska, Germany, and Africa. BMC Microbiol. 2014, 14, 316. [CrossRef]

84. Blekhman, R.; Goodrich, J.K.; Huang, K.; Sun, Q.; Bukowski, R.; Bell, J.T.; Spector, T.D.; Keinan, A.; Ley, R.E.; Gevers, D.; et al. Host genetic variation impacts microbiome composition across human body sites. Genome Biol. 2015, 16, 191. [CrossRef]

85. Herrero, E.R.; Slomka, V.; Bernaerts, K.; Boon, N.; Hernandez-Sanabria, E.; Passoni, B.B.; Quirynen, M.; Teughels, W. Antimicrobial effects of commensal oral species are regulated by environmental factors. J. Dent. 2016, 47, 23-33. [CrossRef] [PubMed]

86. Uehara, Y.; Kikuchi, K.; Nakamura, T.; Nakama, H.; Agematsu, K.; Kawakami, Y.; Maruchi, N.; Totsuka, K. H2O2 produced by viridans group streptococci may contribute to inhibition of methicillin-resistant Staphylococcus aureus colonization of oral cavities in newborns. Clin. Infect. Dis. 2001, 32, 1408-1413. [CrossRef] [PubMed]

87. Al-Shehri, S.; Henman, M.; Charles, B.G.; Cowley, D.; Shaw, P.N.; Liley, H.; Tomarchio, A.; Punyadeera, C.; Duley, J.A. Collection and determination of nucleotide metabolites in neonatal and adult saliva by high performance liquid chromatography with tandem mass spectrometry. J. Chromatogr. B Analyt. Technol. Biomed. Life Sci. 2013, 931, 140-147. [CrossRef] [PubMed]

88. Al-Shehri, S.S.; Knox, C.L.; Liley, H.G.; Cowley, D.M.; Wright, J.R.; Henman, M.G.; Hewavitharana, A.K.; Charles, B.G.; Shaw, P.N.; Sweeney, E.L.; et al. Breastmilk-saliva interactions boost innate immunity by regulating the oral microbiome in early infancy. PLoS ONE 2015, 10, e0135047. [CrossRef]

89. Sweeney, E.L.; Al-Shehri, S.S.; Cowley, D.M.; Liley, H.G.; Bansal, N.; Charles, B.G.; Shaw, P.N.; Duley, J.A.; Knox, C.L. The effect of breastmilk and saliva combinations on the in vitro growth of oral pathogenic and commensal microorganisms. Sci. Rep. 2018, 8 , 15112. [CrossRef]

90. Hurley, E.; Mullins, D.; Barrett, M.P.; O’Shea, C.A.; Kinirons, M.; Ryan, C.A.; Stanton, C.; Whelton, H.; Harris, H.M.B.; O'Toole, P.W. The microbiota of the mother at birth and its influence on the emerging infant oral microbiota from birth to 1 year of age: A cohort study. J. Oral Microbiol. 2019, 11, 1599652. [CrossRef]

91. Lif Holgerson, P.; Esberg, A.; Sjodin, A.; West, C.E.; Johansson, I. A longitudinal study of the development of the saliva microbiome in infants 2 days to 5 years compared to the microbiome in adolescents. Sci. Rep. 2020, 10, 9629. [CrossRef] [PubMed]

92. Kahharova, D.; Brandt, B.W.; Buijs, M.J.; Peters, M.; Jackson, R.; Eckert, G.; Katz, B.; Keels, M.A.; Levy, S.M.; Fontana, M.; et al. Maturation of the oral microbiome in caries-free toddlers: A longitudinal study. J. Dent. Res. 2020, 99, 159-167. [CrossRef] [PubMed]

93. Kennedy, B.; Peura, S.; Hammar, U.; Vicenzi, S.; Hedman, A.; Almqvist, C.; Andolf, E.; Pershagen, G.; Dicksved, J.; Bertilsson, S.; et al. Oral microbiota development in early childhood. Sci. Rep. 2019, 9, 19025. [CrossRef]

94. Kaan, A.M.M.; Kahharova, D.; Zaura, E. Acquisition and establishment of the oral microbiota. Periodontol. 2000 2021, 86, 123-141. [CrossRef] [PubMed] 
95. Al-Shehri, S.S.; Sweeney, E.L.; Cowley, D.M.; Liley, H.G.; Ranasinghe, P.D.; Charles, B.G.; Shaw, P.N.; Vagenas, D.; Duley, J.A.; Knox, C.L. Deep sequencing of the $16 \mathrm{~S}$ ribosomal RNA of the neonatal oral microbiome: A comparison of breast-fed and formula-fed infants. Sci. Rep. 2016, 6, 38309. [CrossRef]

96. Holgerson, P.L.; Vestman, N.R.; Claesson, R.; Ohman, C.; Domellof, M.; Tanner, A.C.; Hernell, O.; Johansson, I. Oral microbial profile discriminates breast-fed from formula-fed infants. J. Pediatr. Gastroenterol. Nutr. 2013, 56, 127-136. [CrossRef]

97. Salli, K.; Soderling, E.; Hirvonen, J.; Gursoy, U.K.; Ouwehand, A.C. Influence of 2'-fucosyllactose and galacto-oligosaccharides on the growth and adhesion of Streptococcus mutans. Br. J. Nutr. 2020, 124, 824-831. [CrossRef]

98. Abranches, J.; Zeng, L.; Kajfasz, J.K.; Palmer, S.R.; Chakraborty, B.; Wen, Z.T.; Richards, V.P.; Brady, L.J.; Lemos, J.A. Biology of oral Streptococci. Microbiol. Spectr. 2018, 6, 6.5.11. [CrossRef]

99. Djais, A.A.; Theodorea, C.F.; Mashima, I.; Otomo, M.; Saitoh, M.; Nakazawa, F. Identification and phylogenetic analysis of oral Veillonella species isolated from the saliva of Japanese children. F1000Research 2019, 8, 616. [CrossRef]

100. Mashima, I.; Theodorea, C.F.; Djais, A.A.; Kunihiro, T.; Kawamura, Y.; Otomo, M.; Saitoh, M.; Tamai, R.; Kiyoura, Y. Veillonella nakazawae sp. nov., an anaerobic Gram-negative coccus isolated from the oral cavity of Japanese children. Int. J. Syst. Evol. Microbiol. 2021, 71, 004583. [CrossRef]

101. Brown, M.M.; Horswill, A.R. Staphylococcus epidermidis-skin friend or foe? PLoS Pathog. 2020, 16, e1009026. [CrossRef] [PubMed]

102. Devang Divakar, D.; Muzaheed; Aldeyab, S.S.; Alfawaz, S.A.; AlKheraif, A.A.; Ahmed Khan, A. High proportions of Staphylococcus epidermidis in dental caries harbor multiple classes of antibiotics resistance, significantly increase inflammatory interleukins in dental pulps. Microb. Pathog. 2017, 109, 29-34. [CrossRef]

103. Jian, C.; Carpen, N.; Helve, O.; de Vos, W.M.; Korpela, K.; Salonen, A. Early-life gut microbiota and its connection to metabolic health in children: Perspective on ecological drivers and need for quantitative approach. EBioMedicine 2021, 69, 103475. [CrossRef] [PubMed]

104. Vatanen, T.; Franzosa, E.A.; Schwager, R.; Tripathi, S.; Arthur, T.D.; Vehik, K.; Lernmark, A.; Hagopian, W.A.; Rewers, M.J.; She, J.X.; et al. The human gut microbiome in early-onset type 1 diabetes from the TEDDY study. Nature 2018, 562, 589-594. [CrossRef] [PubMed]

105. Backhed, F.; Roswall, J.; Peng, Y.; Feng, Q.; Jia, H.; Kovatcheva-Datchary, P.; Li, Y.; Xia, Y.; Xie, H.; Zhong, H.; et al. Dynamics and stabilization of the human gut microbiome during the first year of life. Cell Host Microbe 2015, 17, 690-703. [CrossRef]

106. Garrido, D.; Barile, D.; Mills, D.A. A molecular basis for bifidobacterial enrichment in the infant gastrointestinal tract. Adv. Nutr. 2012, 3, 415S-421S. [CrossRef]

107. Sakanaka, M.; Gotoh, A.; Yoshida, K.; Odamaki, T.; Koguchi, H.; Xiao, J.Z.; Kitaoka, M.; Katayama, T. Varied pathways of infant gut-associated Bifidobacterium to assimilate human milk oligosaccharides: Prevalence of the gene set and its correlation with Bifidobacteria-rich microbiota formation. Nutrients 2019, 12, 71. [CrossRef]

108. Lawson, M.A.E.; O’Neill, I.J.; Kujawska, M.; Gowrinadh Javvadi, S.; Wijeyesekera, A.; Flegg, Z.; Chalklen, L.; Hall, L.J. Breast milk-derived human milk oligosaccharides promote Bifidobacterium interactions within a single ecosystem. ISME J. 2020, 14, 635-648. [CrossRef]

109. LoCascio, R.G.; Ninonuevo, M.R.; Freeman, S.L.; Sela, D.A.; Grimm, R.; Lebrilla, C.B.; Mills, D.A.; German, J.B. Glycoprofiling of bifidobacterial consumption of human milk oligosaccharides demonstrates strain specific, preferential consumption of small chain glycans secreted in early human lactation. J. Agric. Food Chem. 2007, 55, 8914-8919. [CrossRef]

110. Laursen, M.F.; Sakanaka, M.; von Burg, N.; Morbe, U.; Andersen, D.; Moll, J.M.; Pekmez, C.T.; Rivollier, A.; Michaelsen, K.F.; Molgaard, C.; et al. Bifidobacterium species associated with breastfeeding produce aromatic lactic acids in the infant gut. Nat. Microbiol. 2021, 6, 1367-1382. [CrossRef]

111. Ehrlich, A.M.; Pacheco, A.R.; Henrick, B.M.; Taft, D.; Xu, G.; Huda, M.N.; Mishchuk, D.; Goodson, M.L.; Slupsky, C.; Barile, D.; et al. Indole-3-lactic acid associated with Bifidobacterium-dominated microbiota significantly decreases inflammation in intestinal epithelial cells. BMC Microbiol. 2020, 20, 357. [CrossRef] [PubMed]

112. Pannaraj, P.S.; Li, F.; Cerini, C.; Bender, J.M.; Yang, S.; Rollie, A.; Adisetiyo, H.; Zabih, S.; Lincez, P.J.; Bittinger, K.; et al. Association between breast milk bacterial communities and establishment and development of the infant gut microbiome. JAMA Pediatr. 2017, 171, 647-654. [CrossRef] [PubMed]

113. Hill, C.J.; Lynch, D.B.; Murphy, K.; Ulaszewska, M.; Jeffery, I.B.; O'Shea, C.A.; Watkins, C.; Dempsey, E.; Mattivi, F.; Tuohy, K.; et al. Evolution of gut microbiota composition from birth to 24 weeks in the INFANTMET Cohort. Microbiome 2017, 5, 4. [CrossRef]

114. Fallani, M.; Amarri, S.; Uusijarvi, A.; Adam, R.; Khanna, S.; Aguilera, M.; Gil, A.; Vieites, J.M.; Norin, E.; Young, D.; et al. Determinants of the human infant intestinal microbiota after the introduction of first complementary foods in infant samples from five European centres. Microbiology 2011, 157, 1385-1392. [CrossRef]

115. Ray, C.; Kerketta, J.A.; Rao, S.; Patel, S.; Dutt, S.; Arora, K.; Pournami, F.; Bhushan, P. Human milk oligosaccharides: The journey ahead. Int. J. Pediatr. 2019, 2019, 2390240. [CrossRef]

116. Wicinski, M.; Sawicka, E.; Gebalski, J.; Kubiak, K.; Malinowski, B. Human milk oligosaccharides: Health benefits, potential applications in infant formulas, and pharmacology. Nutrients 2020, 12, 266. [CrossRef] [PubMed]

117. Bode, L. Human milk oligosaccharides: Every baby needs a sugar mama. Glycobiology 2012, 22, 1147-1162. [CrossRef]

118. Le Doare, K.; Holder, B.; Bassett, A.; Pannaraj, P.S. Mother's milk: A purposeful contribution to the development of the infant microbiota and immunity. Front. Immunol. 2018, 9, 361. [CrossRef] 
119. Davis, E.C.; Wang, M.; Donovan, S.M. The role of early life nutrition in the establishment of gastrointestinal microbial composition and function. Gut Microbes 2017, 8, 143-171. [CrossRef]

120. Lyons, K.E.; Ryan, C.A.; Dempsey, E.M.; Ross, R.P.; Stanton, C. Breast milk, a source of beneficial microbes and associated benefits for infant health. Nutrients 2020, 12, 1039. [CrossRef]

121. Zivkovic, A.M.; Lewis, Z.T.; German, J.B.; Mills, D.A. Establishment of a milk-oriented microbiota (MOM) in early life: How babies meet their MOMs. Funct. Food Rev. 2013, 5, 3-12.

122. Donovan, S.M.; Wang, M.; Li, M.; Friedberg, I.; Schwartz, S.L.; Chapkin, R.S. Host-microbe interactions in the neonatal intestine: Role of human milk oligosaccharides. Adv. Nutr. 2012, 3, 450S-455S. [CrossRef] [PubMed]

123. Borewicz, K.; Gu, F.; Saccenti, E.; Arts, I.C.W.; Penders, J.; Thijs, C.; van Leeuwen, S.S.; Lindner, C.; Nauta, A.; van Leusen, E.; et al. Correlating infant faecal microbiota composition and human milk oligosaccharide consumption by microbiota of one-month old breastfed infants. Mol. Nutr. Food Res. 2019, 63, e1801214. [CrossRef] [PubMed]

124. Borewicz, K.; Gu, F.; Saccenti, E.; Hechler, C.; Beijers, R.; de Weerth, C.; van Leeuwen, S.S.; Schols, H.A.; Smidt, H. The association between breastmilk oligosaccharides and faecal microbiota in healthy breastfed infants at two, six, and twelve weeks of age. Sci. Rep. 2020, 10, 4270. [CrossRef] [PubMed]

125. Plows, J.F.; Berger, P.K.; Jones, R.B.; Alderete, T.L.; Yonemitsu, C.; Najera, J.A.; Khwajazada, S.; Bode, L.; Goran, M.I. Longitudinal changes in human milk oligosaccharides (HMOs) over the course of 24 months of lactation. J. Nutr. 2021, 151, 876-882. [CrossRef] [PubMed]

126. Minami, J.; Odamaki, T.; Hashikura, N.; Abe, F.; Xiao, J.Z. Lysozyme in breast milk is a selection factor for bifidobacterial colonisation in the infant intestine. Benef. Microbes 2016, 7, 53-60. [CrossRef]

127. Gopalakrishna, K.P.; Hand, T.W. Influence of maternal milk on the neonatal intestinal microbiome. Nutrients 2020, $12,823$. [CrossRef]

128. Gotoh, A.; Katoh, T.; Sakanaka, M.; Ling, Y.; Yamada, C.; Asakuma, S.; Urashima, T.; Tomabechi, Y.; Katayama-Ikegami, A.; Kurihara, S.; et al. Sharing of human milk oligosaccharides degradants within bifidobacterial communities in faecal cultures supplemented with Bifidobacterium bifidum. Sci. Rep. 2018, 8, 13958. [CrossRef]

129. Yu, Z.T.; Chen, C.; Newburg, D.S. Utilization of major fucosylated and sialylated human milk oligosaccharides by isolated human gut microbes. Glycobiology 2013, 23, 1281-1292. [CrossRef]

130. Chia, L.W.; Mank, M.; Blijenberg, B.; Bongers, R.S.; van Limpt, K.; Wopereis, H.; Tims, S.; Stahl, B.; Belzer, C.; Knol, J. Cross-feeding between Bifidobacterium infantis and Anaerostipes caccae on lactose and human milk oligosaccharides. Benef. Microbes 2021, 12, 69-83. [CrossRef]

131. Morrow, A.L.; Ruiz-Palacios, G.M.; Jiang, X.; Newburg, D.S. Human-milk glycans that inhibit pathogen binding protect breastfeeding infants against infectious diarrhea. J. Nutr. 2005, 135, 1304-1307. [CrossRef]

132. Hegar, B.; Wibowo, Y.; Basrowi, R.W.; Ranuh, R.G.; Sudarmo, S.M.; Munasir, Z.; Atthiyah, A.F.; Widodo, A.D.; Supriatmo; Kadim, M.; et al. The role of two human milk oligosaccharides, $2^{\prime}$-fucosyllactose and lacto-n-neotetraose, in infant nutrition. Pediatr. Gastroenterol. Hepatol. Nutr. 2019, 22, 330-340. [CrossRef]

133. Andreas, N.J.; Kampmann, B.; Mehring Le-Doare, K. Human breast milk: A review on its composition and bioactivity. Early Hum. Dev. 2015, 91, 629-635. [CrossRef] [PubMed]

134. Coppa, G.V.; Pierani, P.; Zampini, L.; Carloni, I.; Carlucci, A.; Gabrielli, O. Oligosaccharides in human milk during different phases of lactation. Acta Paediatr. Suppl. 1999, 88, 89-94. [CrossRef] [PubMed]

135. Saben, J.L.; Sims, C.R.; Abraham, A.; Bode, L.; Andres, A. Human milk oligosaccharide concentrations and infant intakes are associated with maternal overweight and obesity and predict infant growth. Nutrients 2021, 13, 446. [CrossRef]

136. Cheema, A.S.; Stinson, L.F.; Rea, A.; Lai, C.T.; Payne, M.S.; Murray, K.; Geddes, D.T.; Gridneva, Z. Human milk lactose, insulin, and glucose relative to infant body composition during exclusive breastfeeding. Nutrients 2021, 13, 3724. [CrossRef] [PubMed]

137. Cheema, A.S.; Lai, C.T.; Dymock, M.; Rae, A.; Geddes, D.T.; Payne, M.S.; Stinson, L.F. Impact of expression mode and timing of sample collection, relative to milk ejection, on human milk bacterial DNA profiles. J. Appl. Microbiol. 2021, 131, 988-995. [CrossRef]

138. Seferovic, M.D.; Mohammad, M.; Pace, R.M.; Engevik, M.; Versalovic, J.; Bode, L.; Haymond, M.; Aagaard, K.M. Maternal diet alters human milk oligosaccharide composition with implications for the milk metagenome. Sci. Rep. 2020, 10, 22092. [CrossRef]

139. Kent, J.C.; Mitoulas, L.R.; Cregan, M.D.; Ramsay, D.T.; Doherty, D.A.; Hartmann, P.E. Volume and frequency of breastfeedings and fat content of breast milk throughout the day. Pediatrics 2006, 117, e387-e395. [CrossRef]

140. Kent, J.C.; Hepworth, A.R.; Sherriff, J.L.; Cox, D.B.; Mitoulas, L.R.; Hartmann, P.E. Longitudinal changes in breastfeeding patterns from 1 to 6 months of lactation. Breastfeed. Med. 2013, 8, 401-407. [CrossRef]

141. Stinson, L.; Hallingstrom, M.; Barman, M.; Viklund, F.; Keelan, J.; Kacerovsky, M.; Payne, M.; Jacobsson, B. Comparison of bacterial DNA profiles in mid-trimester amniotic fluid samples from preterm and term deliveries. Front. Microbiol. 2020, 11, 415. [CrossRef] [PubMed]

142. Cheema, A.S.; Stinson, L.F.; Lai, C.T.; Geddes, D.T.; Payne, M.S. DNA extraction method influences human milk bacterial profiles. J. Appl. Microbiol. 2020, 130, 142-156. [CrossRef] [PubMed]

143. Schloss, P.D.; Westcott, S.L.; Ryabin, T.; Hall, J.R.; Hartmann, M.; Hollister, E.B.; Lesniewski, R.A.; Oakley, B.B.; Parks, D.H.; Robinson, C.J.; et al. Introducing mothur: Open-source, platform-independent, community-supported software for describing and comparing microbial communities. Appl. Environ. Microbiol. 2009, 75, 7537-7541. [CrossRef] [PubMed] 
144. Lenth, R.V. Least-Squares Means: TheRPackagelsmeans. J. Stat. Softw. 2016, 69, 1-33. [CrossRef]

145. R Core Team. R: A Language and Environment for Statistical Computing; R Foundation for Statistical Computing: Vienna, Austria, 2021.

146. Pinheiro, J.; Bates, D.; DebRoy, S.; Sarkar, D.; R Core Team. Nlme: Linear and Nonlinear Mixed Effects Models; R Foundation for Statistical Computing: Vienna, Austria, 2021. 